\title{
AAV-Txnip prolongs cone survival and vision in mouse models of retinitis pigmentosa
}
Yunlu Xue ${ }^{1,2}$, Sean K. Wang ${ }^{1,2,3}$, Parimal Rana ${ }^{1}$, Emma R. West ${ }^{1,3}$, Christin M. Hong ${ }^{1,3}$, Helian Feng $^{4}$, David M. Wu ${ }^{1,2,5}$, Constance L. Cepko ${ }^{1,2,3^{*}}$

1 Department of Genetics, Blavatnik Institute, Harvard Medical School, Boston, MA, USA 02115

2 Department of Ophthalmology, Harvard Medical School, Boston, MA, USA 02115

${ }^{3}$ Howard Hughs Medical Institute, Chevy Chase, MD, USA 20815

${ }^{4}$ Department of Biostatistics, Harvard T.H. Chan School of Public Health, Boston, MA, USA 02115 USA 02114

* email: cepko@genetics.med.harvard.edu 


\section{Abstract}

Retinitis pigmentosa (RP) is an inherited retinal disease, affecting $>20$ million people worldwide. Loss of daylight vision typically occurs due to the dysfunction/loss of cone photoreceptors, the cell type that initiates our color and high acuity vision. Currently, there is no effective treatment for RP, other than gene therapy for a limited number of specific disease genes. To develop a geneagnostic therapy, we screened $\approx 20$ genes for their ability to prolong cone photoreceptor survival in vivo. Here, we report an adeno-associated virus (AAV) vector expressing Txnip, which prolongs the survival of cone photoreceptors and improves visual acuity in RP mouse models. A Txnip allele, C247S, which blocks the association of Txnip with thioredoxin, provides an even greater benefit. Additionally, the rescue effect of Txnip depends on lactate dehydrogenase $b$ (Ldhb), and correlates with the presence of healthier mitochondria, suggesting that Txnip saves RP cones by enhancing their lactate catabolism.

\section{Introduction}

Retinitis pigmentosa (RP) is one of the most prevalent types of inherited retinal diseases, affecting approximately one in 3,000 people (Hartong et al., 2006). In RP, the rod photoreceptors, which initiate night vision, are primarily affected by the disease genes, and degenerate first. The degeneration of cones, the photoreceptors that initiate daylight, color and high acuity vision, then follows, which greatly impacts the quality of life. Currently, one therapy that holds great promise for RP is gene therapy using AAV (Maguire et al., 2019). This approach has proven successful for a small number of genes affecting a few disease families (Cehajic-Kapetanovic et al., 2020). However, due to the number and functional heterogeneity of RP disease genes $(\approx 100$ genes that primarily affect rods (https://sph.uth.edu/retnet/), gene therapy for each RP gene will be logistically and financially difficult. In addition, a considerable number of RP patients do not have an identified disease gene. A disease gene-agnostic treatment aimed at prolonging cone function/survival in the majority of RP patients could thus benefit many more patients. Given that the disease gene is typically not expressed in cones, and thus their death is due to non-autonomous mechanisms that may be in common across disease families, answers to the question of why cones die may provide an avenue to a widely applicable therapy for RP. To date, the suggested mechanisms of cone death include oxidative damage (Komeima et al., 2006; Wellard et al., 2005; Xiong et al., 2015), inflammation (Wang et al., 2020, 2019; Zhao et al., 2015), and a shortage of nutrients (Aït-Ali et al., 2015; Kanow et al., 2017; Punzo et al., 2012, 2009; Wang et al., 2016).

In 2009, we surveyed gene expression changes that occurred during retinal degeneration in four mouse models of RP (Punzo et al., 2009). Those data led us to suggest a model wherein cones starve and die due to a shortage of glucose, which is typically used for energy and anabolic needs in photoreceptors via glycolysis. Evidence of this "glucose shortage hypothesis" was subsequently provided by orthogonal approaches from other groups (Aït-Ali et al., 2015; Wang et al., 2016). These studies have inspired us to test $\approx 20$ genes that might affect the uptake and/or utilization of glucose by cones in vivo in three mouse models of RP (Supplementary Table 1). Only one gene, txnip, had a beneficial effect, prolonging cone survival and visual acuity in these models. Txnip encodes an $\alpha$-arrestin family member protein with multiple functions, including binding to thioredoxin (Junn et al., 2000; Nishiyama et al., 1999), facilitating removal of the glucose transporter 1 (Glut1), from the cell membrane (Wu et al., 2013), and promoting the use of nonglucose fuels (DeBalsi et al., 2014). Because $\alpha$-arrestins are structurally distinct from the visual or $\beta$-arrestins such as Arr3, Txnip is unlikely to bind to opsins or to participate in phototransduction (Hwang et al., 2014; Kang et al., 2015; Puca and Brou, 2014). We tested a number of txnip alleles 
and found that one allele, C247S, which blocks the association of Txnip with thioredoxin (Patwari et al., 2009), provided the greatest benefit. Investigation of the mechanism of Txnip rescue revealed that it required lactate dehydrogenase $b(\mathrm{Ldhb})$, which catalyzes the conversion of lactate to pyruvate. Imaging of metabolic reporters demonstrated an enhanced cytosolic ATP:ADP ratio when the retina was placed in lactate medium. Moreover, mitochondria appeared to be healthier as a result of Txnip addition, but this improvement was not sufficient for cone rescue.

The above observations led to a model wherein Txnip shifts cones from their normal reliance on glucose to enhanced utilization of lactate, as well as marked improvement in mitochondrial structure and function. Analysis of the rescue activity of several additional genes predicted to affect glycolysis, provided support for this model. Finally, as our goal is to rescue cones that suffer not only from metabolic challenges, but also from inflammation and oxidative damage, we tested Txnip in combination with anti-inflammatory and anti-oxidative damage genes, and found additive benefits for cones. These treatments may benefit cones not only in RP, but also in other ocular diseases where similar environmental stresses are present, such as in age related macular degeneration (AMD).

\section{Results}

Txnip prolongs RP cone survival and visual acuity. We delivered genes that might address a glucose shortage and/or mismanagement of metabolism in a potentially glucose-limited environment. To this end, twelve AAV vectors were constructed to test genes singly or in combination for an initial screen (Extended Data Fig. 1e). Subsequently, an additional set of AAV vectors were made based upon the initial screen results, as well as other rationales, to total 20 genes tested in all (Supplementary Table 1). Most of these vectors carried genes to augment the utilization of glucose, such as hexokinases (Hk1 and Hk2), phosphofructokinase (Pfkm) and pyruvate kinase (Pkm1 and Pkm2). Each AAV vector used a cone-specific promoter, which was previously found to be non-toxic at the doses used in this study (Xiong et al., 2019). An initial screen was carried out in $r d 1$ mice, which harbor a null allele in the rod-specific gene, Pde6b. This strain has a rapid loss of rods, followed by cone death. The vectors were subretinally injected into the eyes of neonatal $r d 1$ mice, in combination with a vector using the human red opsin (RedO) promoter to express a histone 2B-GFP fusion protein (AAV-RedO-H2BGFP). The H2BGFP provides a very bright cone-specific nuclear labelling, enabling automated quantification. As a control, eyes were injected with AAV-RedO-H2BGFP alone. Rd1 cones begin to die at $\approx$ postnatal day 20 (P20), when almost all rods have died (Extended Data Fig. 1a). The number of $r d 1$ cones was quantified by counting the H2BGFP+ cells using a custom-made MATLAB program (Fig. 1a and Extended Data Fig. 1c). Only cones within the central region of the retina were counted, since RP cones in the periphery die much later (Hartong et al., 2006; Punzo et al., 2009). Among the twelve tested vectors, and six of their combinations, we found that only Txnip led to an increase in P50 rd1 cones. The effects were likely on cone survival, as it did not change the number of cones at P20 prior to their death (Fig. 1a,b, and Extended Data Fig. 1c,e). The level of Txnip rescue in P50 rd1 cones was comparable to using AAV with a CMV promoter to express a transcription factor, Nrf2, that regulates anti-oxidation pathways and reduces inflammation as we found previously (Xiong et al., 2015) (Extended Data Fig. 1e). One combination led to a reduction in cone survival, that of Hk1 plus Pfkm (Extended Data Fig. 1e).

Our initial screen used the RedO promoter to drive Txnip expression. To evaluate a different conespecific promoter, Txnip also was tested using a newly described cone-specific promoter, SynPVI (Jüttner et al., 2019). This promoter also led to prolonged cone survival (Extended Data Fig. 1e). To explore whether Txnip gene therapy is effective beyond $r d 1$, it was tested in $r d 10$ mice, which carry a missense Pde6b mutation, and in $r h o^{-/}$mice, which carry a null allele in a rod-specific 
119 protein, rhodopsin. Prolonged cone survival was observed in both strains (Fig. 1a,b). To determine 
171 dehydrogenase a (Ldha, encoded by ldha gene), converts pyruvate to lactate (Eventoff et al.,

172 1977). We found that Txnip rescue was significantly decreased by any one of three Ldhb shRNAs

173 (siLdhb) or by overexpression of Ldha (Fig. 3a,b and Extended Data Fig. 3). We also tested the rescue effect of Txnip plus an shRNA against Oxct1 (siOxct1), a critical enzyme for ketolysis (Zhang and Xie, 2017), or against Cpt1a (siCpt1a), a component for lipid transporter that is rate limiting for $\beta$-oxidation (Shriver and Manchester, 2011). These shRNAs, tested singly or in combination, did not reduce the effectiveness of Txnip rescue (Fig. 3c). Taken together, these data support the use of lactate, but not ketones or lipids, as a critical alternative fuel for cones when Txnip is overexpressed.

Txnip improves the ATP:ADP ratio in RP cones in the presence of lactate. If the improved survival of cones following Txnip overexpression is due to improved utilization of non-glucose fuels, cones might show improved mitochondrial metabolism. To begin to examine the metabolism of cones, we first attempted to perform metabolomics of cones with and without Txnip. However, so few cones are present in these retinas that we were unable to achieve meaningful results. An alternative assay was conducted to measure the ratio of ATP to ADP using a genetically-encoded fluorescent sensor (GEFS). AAV was used to deliver PercevalHR, an ATP:ADP GEFS (Tantama et al., 2013), to $r d 1$ cones with and without AAV-Txnip. The infected P20 rd1 retinas were explanted and imaged in three different types of media to measure the cone cytosolic ratio of ATP:ADP. Txnip increased the ATP:ADP ratio (i.e. higher $\mathrm{F}_{\text {PercevalHR }}{ }^{488: 405}$ ) of $r d 1$ cones in lactateonly or pyruvate-only media. Consistent with the role of Txnip in removing Glut1 from the plasma membrane, Txnip treated cones had a lower ATP:ADP ratio (i.e. lower $\mathrm{F}_{\text {PercevalHR }}{ }^{488: 405}$ ) in high glucose medium (Fig. 4a,b). To further probe whether intracellular glucose was reduced after overexpression of Txnip (Wu et al., 2013), a glucose sensor iGlucoSnFR was used (Keller et al., 2019). This sensor showed reduced intracellular glucose in Txnip-treated cones (Extended Data Fig. 4a,b). Because the fluorescence of GEFS may be also subject to environmental $\mathrm{pH}$, we used a pH sensor, pHRed (Tantama et al., 2011), to determine if the changes of PercevalHR and iGlucoseSnFR were due to a change in $\mathrm{pH}$, and it showed no significant $\mathrm{pH}$ change (Extended Data Fig. 4c,d). We also found that lactate, but not pyruvate, utilization by Txnip-treated cones was critically dependent upon Ldhb for ATP production, as introduction of siLdhb abrogated the increase in ATP:ADP in Txnip-treated cones (Fig. 4c). Furthermore, in correlation with improved cone survival by Txnip.C247S compared to wt Txnip (Fig. 2b), cones had a higher ATP:ADP ratio in lactate medium when Txnip.C247S was used relative to wt Txnip (Fig. 4e). Similarly, in correlation with no survival benefit when treated with Txnip.S308A (Fig. 2b), there was no difference in the ATP:ADP ratio when Txnip.S308A was used, relative to control, in lactate medium (Fig. 4e).

Txnip improved RP cone mitochondrial gene expression, size, and function. To further probe the mechanism(s) of Txnip rescue, we first tested if all of the benefits of Txnip were due to Txnip's effects on Ldhb. Ldhb was thus overexpressed alone or with Txnip. Ldhb alone did not prolong cone survival, nor did it increase the Txnip rescue (Extended Data Fig. 6e). An additional experiment was carried out to investigate if there might be a shortage of the mitochondrial pyruvate carrier, which could limit the uptake of pyruvate into the mitochondria of photoreceptors for ATP synthesis (Grenell et al., 2019). The pyruvate carrier, which is a dimer encoded by mpc1 and $m p c 2$ genes, thus was overexpressed, but did not prolong $r d 1$ cone survival (Extended Data Fig. 6c). To take a less biased approach, the transcriptomic differences between Txnip-treated and control RP cones were characterized. H2BGFP labeled RP cones were isolated by FACS-sorting at an age when cones were beginning to die, and RNA-sequencing was performed (Extended Data Fig. 5a). Data were obtained from two RP strains, $r d 1$ and $r h o^{-1}$. By comparing the differentially expressed genes in common between the two strains, relative to control, seven genes were seen to be upregulated and 17 were downregulated (Supplementary Table 2). Three of the seven upregulated genes were mitochondrial electron transport chain (ETC) genes. The 
223 upregulation of these three ETC genes in Txnip-treated $r d 1$ cones was confirmed by ddPCR

224 (Extended Data Fig. 5b).

225

226

227

228

229

230

231

232

233

234

235

236

237

238

239

240

241

242

243

244

245

246

247

248

249

250

251

252

253

254

255

256

257

258

259

260

261

262

263

264

265

266

267

268

269

270

271

272

273

The finding of upregulated ETC genes in Txnip-treated cones suggested effects on mitochondria, and thus the morphology of Txnip-treated mitochondria in RP cones was examined by electron microscopy (EM). There was an increase in mitochondrial size by Txnip treatment, with a greater increase in size following treatment with Txnip.C247S (Fig. 5a,b). Mitochondrial membrane potential $(\Delta \Psi \mathrm{m})$ activity, a reflection of mitochondrial ETC function, was also examined using JC-1 dye staining of freshly explanted Txnip-treated P20 rd1 retinas (Reers et al., 1995). Both Txnip and Txnip.C247S increased the ratio of J-aggregates:JC1-monomers (Fig. 5c,d), indicating an increased $\Delta \Psi \mathrm{m}$ and/or a greater number/size of mitochondria with a high $\Delta \Psi \mathrm{m}$ following Txnip overexpression. This finding was further investigated in vivo using infection by an AAV encoding mitoRFP, which only accumulates in mitochondria with a high $\Delta \Psi \mathrm{m}$ (Brodier et al., 2020; Hood et al., 2003). Compared to the control cones without Txnip treatment, the intensity of mitoRFP was higher in P20 rd1 cones treated with Txnip (Extended Data Fig. 5c,d).

A previous study identified 15 proteins that interact with Txnip.C247S (Forred et al., 2016). Among these interactors was Parp1, which can negatively affect mitochondria through deleterious effects on the mitochondrial genome (Hocsak et al., 2017; Szczesny et al., 2014), as well as have effects on inflammation and other cellular pathways (Fehr et al., 2020). Due to the similarities between the effects of Txnip addition and of Parp1 inhibition on mitochondria, Parp1 was tested for a potential role in Txnip-mediated rescue. Parp1 expression was first examined by immunohistochemistry and found to be enriched in cone inner segments, which are packed with mitochondria (Hoang et al., 2002), and in cone nuclei (Extended Data Fig. 5g). Interestingly, these are the same locations where a GFP-Txnip fusion protein was found (Extended Data Fig. 1b). To test for a role of Parp1, parp $1^{-/-}$mice were bred to $\mathrm{rd} 1$ mice, and their cone mitochondria were examined by EM and mitoRFP. Parp 1/- rd1 cones possessed larger mitochondria (Extended Data Fig. 5h,i) and higher mitoRFP signals than cones from parp ${ }^{1 /+} r d 1$ controls. Addition of Txnip.C247S to parp $1^{-/-} r d 1$ cones did not alter the mitoRFP signals (Fig. 5e,f). However, when Txnip.C247S was added to parp $1^{-/-} r d 1$ retinas, cone survival was similar to that of Txnip.C247S-treatred $p a r p 1^{+/+} r d 1$ retinas, showing that Txnip-mediated survival does not require Parp1 (Fig. $5 g, h$ ).

The discordance between improved mitochondria and cone survival in these experiments suggested that mitochondrial improvement alone is not sufficient to prolong cone survival. This is consistent with the observations from treatment with Txnip.S308A, as well as Txnip + siLdhb, both of which failed to prolong $r d 1$ cone survival despite improvements in mitochondria (Fig. 2a,b,5a,b,c,d, and Extended Data Fig. 5c,d,e,f). To test if improved cone survival requires both mitochondrial improvement and enhanced lactate catabolism, we delivered Ldhb to parp ${ }^{1 /-}$ rd1 cones. A small but significant improvement in cone survival was observed (Fig. 5i,j).

Txnip enhances $\mathrm{Na}^{+} / \mathrm{K}^{+}$pump function and cone opsin expression. The results above suggest that Txnip may prolong RP cone survival by enhancing lactate catabolism via Ldhb, which may lead to greater ATP production by the oxidative phosphorylation (OXPHOS) pathway. Cone photoreceptors are known to require high levels of ATP to maintain their membrane potential, relying primarily upon the $\mathrm{Na}^{+} / \mathrm{K}^{+}$ATPase pump (Ingram et al., 2020). To investigate whether Txnip affects the function of the $\mathrm{Na}^{+} / \mathrm{K}^{+}$pump in RP cones, freshly explanted P20 rd1 retinas were treated with $\mathrm{RH} 421$, a fluorescent small-molecule probe for $\mathrm{Na}^{+} / \mathrm{K}^{+}$pump function (Fedosova et al., 1995). Addition of Txnip improved $\mathrm{Na}^{+} / \mathrm{K}^{+}$pump function of these cones in lactate medium as reflected by an increase in RH421 fluorescence (Fig. 6a,b), consistent with Txnip enabling greater utilization of lactate. In RP cones, it is also known that protein expression of cone opsin is downregulated, postulated to be due to insufficient energy supply (Punzo et al., 2009). Compared to 
274 control, greater anti-opsin staining was observed in Txnip-treated $r d 1$ cones at P50 (Fig. 6c),

\section{Dominant-negative HIF1a improves RP cone survival. If improved lactate catabolism and} OXPHOS are at least part of the mechanism of Txnip rescue, RP cone survival might be promoted by other molecules serving similar functions. HIF1 $\alpha$ can upregulate the transcription of glycolytic genes (Majmundar et al., 2010). Increased glycolytic enzyme levels might push RP cones to rely on glucose, rather than lactate, to their detriment if glucose is limited. To investigate whether HIF1 $\alpha$ might play a role in cone survival, a wt and a dominant-negative HIF1 $\alpha$ (dnHIF1 1 ) allele (Jiang et al., 1996) were delivered to $r d 1$ retinas using AAV. A target gene of HIF1a, vegf, which might improve blood flow and thus nutrient delivery, also was tested. The dnHIF1 $\alpha$ increased $r d 1$ cone survival, while wt HIF1 $\alpha$ and Vegf each decreased cone survival (Fig. 7a,b, Extended Data Fig. 6d,e).

Txnip effects on Glut1 levels in the RPE and cone survival. Several lines of evidence support the hypothesis that RP cones do not have sufficient glucose to satisfy their needs via glycolysis (Chinchore et al., 2019; Kanow et al., 2017; Punzo et al., 2012, 2009; Wang et al., 2016). To determine if retention of glucose by the RPE might underlie a glucose shortage for cones (Kanow et al., 2017; Wang et al., 2016), we attempted to reprogram RPE metabolism to a more "OXPHOS" and less "glycolytic" status by overexpressing Txnip or dnHIF1a with an RPE-specific promoter, the Best1 promoter (Esumi et al., 2009). The goal was to increase lactate consumption in the RPE, thus freeing up more glucose for delivery to cones. However, no RP cone rescue was observed (Extended Data Fig. 6b), possibly due to a clearance of Glut1 from the surface of cells, which would create a glucose shortage for both the RPE and the cones (Swarup et al., 2019) (Extended Data Fig. 6a). To examine the level of Glut1 in the RPE following introduction of wt Txnip, or Txnip.C247S.LL351\&352AA, which should prevent efficient removal of Glut1, immunohistochemistry for Glut1 was carried out. This assay showed that AAV-Best1Txnip.LL351\&352AA did result in less clearance of Glut1 from the surface of the RPE (Extended Data Fig. 6a) relative to wt Txnip. Txnip.C247S.LL351\&352AA was then tested for $r d 1$ cone rescue, where it was found to improve cone survival (Fig. 7a,b), in keeping with the model that the RPE retains glucose to the detriment of cones in RP.

Combination of Txnip.C247S with other rescue genes provides an additive effect. Finally, as our goal is to provide effective, generic gene therapy for RP, and potentially other diseases that affect photoreceptor survival, we used combinations of AAVs that encode genes that we have previously shown prolong RP cone survival and vision. The combination of Txnip.C247S expression in cones, with expression of Nrf2, a gene with anti-oxidative damage and antiinflammatory activity, in the RPE, provided an additive effect on cone survival relative to either gene alone (Fig. 8a,b). This combination also preserved the RP cone outer segments, which is the structure packed with opsin for photon detection, and reduced the mislocalization of opsin to the plasma membrane (Fig. 8c). An interesting phenotype that is especially prominent in the FVB rd1 strain is that of "craters" in the photoreceptor layer. These are areas of circumscribed cone death that are obvious when the retina is viewed as a flat-mount. AAV-Best1-Nrf2 alone suppressed the formation of these craters (Wu et al., n.d.), while AAV-RedO-Txnip did not, despite the fact that AAV-RedO-Txnip.C247S provides the most robust RP cone rescue that we have seen (Fig. 2a,6f,6h). An additional combination that was tested was AAV-RedO-Txnip.C247S with AAVRedO-Tgfb1, an anti-inflammatory gene (Wang et al., 2020). This combination did not improve cone survival beyond that of Txnip alone, but almost completely eliminated the craters (Fig. 8d,e). In addition, we tried other genes in combination with wt Txnip, but did not observe any obvious improvement over Txnip alone (Extended Data Fig. 6e). 


\section{Discussion}

Photoreceptors have been characterized as being highly glycolytic, even under aerobic conditions, as originally described by Otto Warburg (Warburg, 1925). Glucose appears to be supplied primarily from the circulation, via the RPE, which has a high level of Glut1 (Gospe et al., 2010). Photoreceptors, at least rods, carry out glycolysis to support anabolism, to replace their outer segments (Chinchore et al., 2017), and contribute ATP, to run their ion pumps (Okawa et al., 2008). If glucose becomes limited, as has been proposed to occur in RP, cones may have insufficient fuel for their needs. To explore whether we could develop a therapy to address some of these metabolic shortcomings in RP, we delivered many different types of genes that might alter metabolic programming. From these, Txnip had the strongest benefit on cone survival and vision (Fig. 1 and Extended Data Fig. 1). This was surprising as Txnip has been shown to inhibit glucose uptake, by binding to and aiding in the removal of Glut1 from plasma membrane, and it inhibits the anti-oxidation proteins, the thioredoxins, again by direct binding. The results with Txnip in its wt form, and from the study of several mutant alleles, provide some insight into how it might benefit cones. The Txnip.C247S allele prevents binding to thioredoxins, and gave enhanced cone survival relative to wt Txnip (Fig. 2 and Extended Data Fig. 2). We speculate that, by being free of this interaction, the C247S mutant protein may be more available for other Txnip-mediated activities. In addition, thioredoxin may be made more available for its role in fighting oxidative damage.

The mechanisms by which Txnip might benefit cones are not fully known, but a study of Txnip's function in skeletal muscle suggested that it plays a role in fuel selection (DeBalsi et al., 2014). If glucose is limited in RP, then cones may need to switch from a reliance on glucose and glycolysis to an alternative fuel(s), such as ketones, fatty acids, amino acids, or lactate. Cones express oxct1 mRNA (Shekhar et al., 2016), which encodes a critical enzyme for ketone catabolism, suggesting cones are capable of ketolysis. In addition, a previous study showed that lipids might be an alternative energy source for cones by $\beta$-oxidation (Joyal et al., 2016). It is likely that cones can use these alternative fuels to meet their intense energy demands (Ingram et al., 2020) (Fig. 6,7). However, the Txnip rescue did not depend on ketolysis or $\beta$-oxidation (Fig. 3). Due to the diversity of amino acid catabolic pathways, we did not study whether these pathways were required for Txnip's rescue effect. However, we did discover that Ldhb, which converts lactate to pyruvate, was required. This is an interesting switch, as photoreceptors normally have high levels of Ldha, and produce lactate (Chinchore et al., 2017). An important factor in the reliance on Ldhb could be the availability of lactate, which is highly available from serum (Hui et al., 2017). Lactate could be transported via the RPE and/or Müller glia, and/or the internal retinal vasculature which comes in closer proximity to cones after rod death. Ketones are usually only available during fasting, and lipids are hydrophobic molecules which are slow to be transported across the plasma membranes. Moreover, lipids are required to rebuild the membrane-rich outer segments, and thus might be somewhat limited. Ldhb is not sufficient, however, to delay RP cone degeneration, as its overexpression did not promote RP cone survival.

Txnip-treated RP cones also had larger mitochondria with a greater membrane potential, and likely were able to use the pyruvate produced by Ldhb for greater ATP production via OXPHOS. Indeed, Txnip-treated cones had an enhanced ATP:ADP ratio (Fig. 4). However, healthier mitochondria were not sufficient to prolong RP cone survival. Txnip.S308A led to larger mitochondria than control mitochondria, brighter JC-1 staining and mitoRFP signals, which are indicators of better mitochondrial health, but this allele did not induce greater cone survival (Fig. 5 and Extended Data Fig. 5). Moreover, as Txnip has been shown to interact with Parp1, which can negatively affect mitochondria, we investigated if Parp1 knock-out mice might have cones that survive longer in RP. Indeed, the Parp1 knock-out mitochondria appeared to be healthier, but Parp1 knock-out retinas did not have better RP cone survival than Parp1-wt rd1 retinas. In addition, cone rescue by Txnip was not changed in the Parp1 knock-out retinas. 
The well-described effects of Txnip on the removal of Glut1 from the cell membrane might seem at odds with the promotion of cone survival. It could be that removal of Glut1 from the plasma membrane of cones forces the cones to choose an alternative fuel, such as lactate, and perhaps others too. Interestingly, as Glut1 knock-down was not sufficient for cone survival, Txnip must not only lead to a reduction in membrane localized Glut1, but also potentiate a fuel switch, via an unknown mechanism(s) that at least involves an increase of Ldhb activity. A reduction in glycolysis might also lead to a fuel switch. Introduction of dnHIF1a, which should reduce expression of glycolytic enzymes, also benefitted cones, while introduction of wt HIF1a did not (Fig. 7). HIF1a has many target genes, and may alter pathways in addition to that of glycolysis, thus also potentiating a fuel switch once glycolysis is down regulated. An additional finding supporting the notion that the level of glycolysis is important for cone survival was the observation that AAV-Pfkm plus AAV-Hk1 led to a reduction in cone survival (Extended Data Fig. 1e). Phosphorylation of glucose by Hk1 followed by phosphorylation of fructose-6-phosphate by the Pfkm complex commits glucose to glycolysis at the cost of ATP. These AAVs may have promoted the flux of glucose through glycolysis, which may have inhibited a fuel switch, and/or depleted the ATP pool, e.g. if downstream glycolytic intermediates were used for anabolic needs so that ATP production by glycolysis did not occur.

The observations described above suggest that at least two different pathways are required for the promotion of cone survival by Txnip (Extended Data Fig. 8). One pathway requires lactate utilization via Ldhb, but as Ldhb was not sufficient, another pathway is also required. As greater mitochondrial health was observed following Txnip treatment, a second pathway may include the effects on mitochondria. This notion is supported by the observation that the addition of Ldhb to

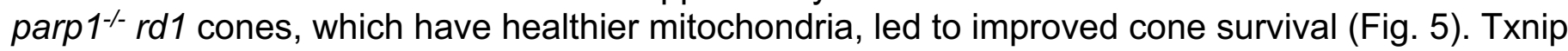
alone may be able to promote cone health by impacting both lactate catabolism and mitochondrial health. There may be additional pathways required as well.

The effects of Txnip alleles expressed only in the RPE provide some support for the hypothesis that the RPE transports glucose to cones for their use, while primarily using lactate for its own needs (Kanow et al., 2017; Swarup et al., 2019). Lactate is normally produced at high levels by photoreceptors in the healthy retina. When rods, which are $97 \%$ of the photoreceptors, die, lactate production goes down dramatically. The RPE might then need to retain glucose for its own needs. Introduction of an allele of Txnip, C247S.LL351\&352AA, to the RPE provided a rescue effect for cones, while introduction of the wt allele of Txnip to the RPE did not. The LL351\&352AA mutations lead to a loss of efficiency of the removal of Glut1 from the plasma membrane, while the C247S mutation might create an even less glycolytic RPE. The combination of these mutations might then allow more glucose to flow to cones. The untreated RP cones seem to be able to use glucose at a high concentration for ATP production, at least in freshly explanted retinas (Fig. 4a). These findings are also consistent with the reported mechanism for cone survival promoted by RdCVF, a factor that is proposed to improve glucose uptake by RP cones, which might be important if glucose is present in low concentration due to retention by the RPE (Aït-Ali et al., 2015; Byrne et al., 2015).

As cones face multiple challenges in the degenerating RP retina, we tested Txnip in combination with genes that we have found to promote cone survival via other mechanisms. The combination of Txnip with vectors fighting oxidative stress (AAV-Best1-Nrf2) or inflammation (AAVRedO-Tgfb1) supported greater cone survival than any of these treatments alone. These combinations utilize cell type-specific promoters, reducing the chances of side effects from global expression of these genes. Of note, the Nrf2 expression was limited to the RPE, yet was additive for cone survival. This finding is in keeping with the interdependence of photoreceptors and the RPE, which is undoubtably important not only in a healthy retina, but in disease as well. 
430

731

732

733

734

735

436

737

738

439

440

741

442

743

744

745

746

747

748

749

450

451

752

453

454

755

456

757

758

459

760

761

762

763

764

765

766

467

468

469

470

471

772

473

474

475

476

477

478

479

780

781

\section{Methods}

\section{Animals}

rd1 mice were the albino FVB strain, which carries the Pde6b ${ }^{\text {rd1 }}$ allele (MGI: 1856373). BALB/c, CD1, and FVB mice were purchased from Charles River Laboratories. Due to availability, part of the FVB mice were purchased from Taconic, and we did not notice any difference between the two sources in terms of cone degeneration rate. C57BL/6J, rd10, and parp $1^{-/}$mice were purchased from The Jackson Laboratories and bred in house. We crossed the parp $1^{-1-}$ mice with FVB mice to generate homozygous parp $1^{-/-} r d 1$ and $p a r p 1^{+/+} r d 1$ mice. Genotyping of these mice was done by Transnetyx (Cordova, TN). The rho $^{-/-}$mice were provided from by Lem (Tufts University, MA) (Lem et al., 1999).

\section{AAV vector design, authentication, and preparation}

Detailed information of all AAVs used in this study is listed in Supplementary Table 1, along with the authentication information. cDNAs of mouse txnip, hif1a, hk2, Idha, Idhb, slc2a1, bsg1, cpt1a, oxct1, mpc1 and $m p c 2$, and human nrf2, were purchased from GeneCopeia (Rockville, MD). Mouse vegf164 cDNA (Robinson and Stringer, 2001) was synthesized by Integrated DNA Technologies (Coralville, lowa). We obtained the following plasmids as gifts from various depositors through Addgene (Watertown, MA): hk1, pfkm and pkm2 (William Hahn \& David Root; \#23730, \#23728, \#23757), pkm1 (Lewis Cantley \& Matthew Vander Heiden; \#44241), H2B-GFP (Geoff Wahl; \#11680), mitoRFP (i.e. DsRed2-mito, Michael Davidson; \#55838), GFP-Txnip (Clark Distelhorst; \#18758), W3SL (Bong-Kiun Kaang; \#61463), 3xFLAG (Thorsten Mascher, \#55180), PercevalHR and pHRed (Gary Yellen; \#490820, \#31473). The cDNA of mouse RdCVF was a gift from Leah Byrne and John Flannery (UC Berkeley, CA). iGlucoSnFR was provided under a Material Transfer Agreement by Jacob Keller and Loren Looger (Janelia Research Campus, VA). RedO promoter was provided as a gift, and SynPVI and SynP136 promoters were provided under a Material Transfer Agreement, from Botond Roska (IOB, Switzerland). The Best1 promoter was synthesized by lab member, Wenjun Xiong, using Integrated DNA Technologies based on literature (Esumi et al., 2009). Mutated Txnip, dominant-negative HIF1a (Jiang et al., 1996) and RO1.7 promoter (Ye et al., 2016) were created from the corresponding wildtype plasmids in house using Gibson assembly.

All of the new constructs in this study were cloned using Gibson assembly. For example, AAVRedO-Txnip was cloned by replacing the EGFP sequence of AAV-RedO-EGFP at the Notl/HindIII sites, with the Txnip sequence, which was PCR-amplified from the cDNA vector adding two 20-bp overlapping sequences at the 5'- and 3'-ends. All of the AAV plasmids were amplified using Stbl3 E. coli (Thermo Fisher Scientific). The sequences of all AAV plasmids were verified with directed sequencing and restriction enzyme digestion. The key plasmids were triple-verified with NextGeneration complete plasmid sequencing (MGH CCIB DNA Core), which is able to capture the full sequence of the ITR regions. The genome sequence of critical AAVs (i.e. AAV8-RedOTxnip.C247S and AAV8-RedO-Txnip.S308A) were quadruple-verified with PCR and directed sequencing.

All of the vectors were packaged in recombinant AAV8 capsids using HEK293T cells and purified with iodixanol gradient as previously described (Grieger et al., 2006; Xiong et al., 2015). The titer of each AAV batch was determined using protein gels, comparing viral band intensities with a previously established AAV standard. The concentration of our AAV production usually ranged from $2 \times 10^{12}$ to $3 \times 10^{13} \mathrm{gc} / \mathrm{mL}$. Multiple batches of key AAV vectors (e.g. 4 batches of AAV8RedO-Txnip, and 3 batches of AAV-RedO-siLdhb ${ }^{(\# 2)}$ ) were made and tested in vivo to avoid any unknown batch effects. 
shRNA they were provided as three or four distinct sequences for each gene, driven by the $\mathrm{H} 1$ or $\mathrm{U} 6$ promoter. The knockdown efficiency of these candidate shRNA sequences was tested by cotransfecting with CAG-TargetGene-IRES-d2GFP vector in HEK293T cells as previously described (Matsuda and Cepko, 2007; Wang et al., 2014). The GFP fluorescence intensity served as a fast and direct read out of the knockdown efficiency of these shRNAs. Using this method, we selected the following sense strand sequences to knockdown the targeted genes (Extended Data Fig. 811): siLdhb(\#2) 5'-CCATCATCGTGGTTTCCAACC-3'; sildhb(\#1) 5'GCAGAGAAATGTCAACGTGTT-3'; siLdhb(\#3) 5'-GCCGATAAAGATTACTCTGTG-3'; siSIc2a1 (\#a) 5'-GGTTATTGAGGAGTTCTACAA-3'; siOxct1 1\#c) 5'-GGAAACAGTTACTGTTCTCCC-3'; siCpt1 a ${ }^{(\#) ~ 5 '-G C A T A A A C G C A G A G C A T T C C T-3 ' ; ~ a n d ~ s i N C ~(n o n-t a r g e t i n g ~ s c r a m b l e d ~ c o n t r o l ~}$ sequence) 5'-GCTTCGCGCCGTAGTCTTA-3'. We cloned the entire hairpin sequence (including a 6-bp 5'-end lead sequence 5'-gatccg-3', a 7-bp loop sequence 5'-TCAAGAG-3' between sense and antisense strands, and a > 7-bp 3'end sequence 5'-tttttg-3') and packaged them into AAV8RedO-shRNA using Gibson assembly as described above. To maximize the knockdown efficacy using a Pol II promoter in AAV (Giering et al., 2008), no extra base pair was kept between the RedO promoter and the 5'-end lead sequence of shRNAs. Due to the lack of an adequate Ldhb antibody, we confirmed the in vivo Ldhb knockdown efficiency of all three AAV8-RedO-siLdhb vectors by co-injection with an AAV8-Ldhb-3xFLAG vector into wildtype mouse eyes and detection for FLAG immunofluorescence as described in the Histology section below (Extended Data Fig. 3a).

Subretinal injection

On the day of birth (P0), $\approx 1 \times 10^{9} \mathrm{vg} /$ eye of AAV was injected into the eyes of pups as previously described(Matsuda and Cepko, 2007; Xiong et al., 2015). For all experiments in which cones were quantified, and to provide a means to trace infection (e.g. for immunohistochemistry), $2.5 \times 10^{8}$ vg/eye of AAV8-RedO-H2BGFP was co-injected with other AAVs, or alone as a control. For all other experiments, such as FACS sorting and ex vivo live-imaging, 1 x $10^{9} \mathrm{vg} / \mathrm{eye}$ of AAV8SynP136-H2BGFP was co-injected.

\section{Photopic visual acuity measured for optomotor response}

The photopic optomotor response of mice were measured using the OptoMotry System (CerebralMechanics) at a background light of $\approx 70 \mathrm{~cd} / \mathrm{m}^{2}$ as previously described (Xiong et al., 2019). The contrast of the grates was set to be $100 \%$, and temporal frequency was $1.5 \mathrm{~Hz}$. The threshold of mouse visual acuity (i.e. maximal spatial frequency) was tested by an examiner without knowledge of the injected or the treatment group. During each test, the direction of movement of the grates (i.e. clockwise or counterclockwise) was randomized, and the spatial frequency of each testing episode was determined by the software. Without knowing the spatial frequency of the moving grates, the examiner reported either "yes" or "no" to the system until the threshold of acuity was determined by the software.

\section{Histology}

Mice were euthanized with $\mathrm{CO}_{2}$ and cervical dislocation, and the eye was enucleated. For flatmounts, retinas were separated from the rest of the eye using a dissecting microscope and were fixed in $4 \%$ paraformaldehyde solution for 30 minutes. The retinas were then flat mounted on a glass slide and coverslip. For H2BGFP labeled cone imaging, we used a Keyence microscope with a 10x objective (Plan Apo Lamda 10x/0.45 Air DIC N1) and GFP filter box (OP66836). 
534 For cone opsin antibody staining in whole-mount retinas, after fixation, retinas were blocked for 1 hour in PBS with 5\% normal donkey serum and 0.3\% Triton X-100 at room temperature. After blocking, retinas were incubated with a mixture of 1:200 anti-s-opsin antibody (AB5407, EMD Millipore) and 1:600 anti-m-opsin antibody (AB5405, EMD Millipore) in the same blocking solution overnight at $4{ }^{\circ} \mathrm{C}$, followed by secondary donkey-anti-rabbit antibody staining (1:1000, Alexa Fluor 594 ) at room temperature for 2 hours, then flat-mounted on a glass slide and coverslip.

For frozen sections, whole eyes were fixed in $4 \%$ paraformaldehyde solution for 2 hours at room temperature, followed by removing the cornea, lens and iris. Then the eye cups went through $15 \%$ and $30 \%$ sucrose gradient to dehydrate at room temperature, followed by overnight incubation in $1: 130 \%$ sucrose and Tissue-Tek® O.C.T. solution at $4{ }^{\circ} \mathrm{C}$. Eye cups were embedded in a plastic mold, frozen in a $-80^{\circ} \mathrm{C}$ freezer, and cut into $20 \mu \mathrm{m}$ or $12 \mu \mathrm{m}$ thin radial cross-sections which were placed on glass slides. Antibody staining was done similarly to whole-mounts as described above and previously (Wang et al., 2014). PBS with $0.1 \%$ Triton X-100, $5 \%$ normal donkey serum and $1 \%$ bovine serum albumin (BSA) was used as the blocking solution, except for FLAG detection (10\% donkey serum and 3\% BSA). Glut1 (encoded by slc2a1 gene) antibody (GT11-A, Alpha Diagnostics) was used at 1:300 dilution, Parp1 antibody (ab227244, Abcam) was used at 1:300 dilution, GFP antibody (ab13970, Abcam) was used at 1:1000 dilution to detect GFP-Txnip, and FLAG antibody (ab1257, Abcam) was used at 1:2000 based on a previous study(Ferrando et al., 2015). If applicable, 1:1000 PNA (CY5 or Rhodamine labeled) for cone extracellular matrix labeling, and 1:1000 DAPI were used to co-stain with secondary antibodies. Stained sections were imaged with a confocal microscope (LSM710, Zeiss) using 20x or 63x objectives (Plan Apo 20x/0.8 Air DIC II, or Plan Apo 63X/1.4 Oil DIC III).

\section{Automated RP cone counting}

The cone-H2BGFP images of whole flat-mounted retinas were first analyzed in Image $J$ to acquire the diameter and the center parameters of the sample. We used a custom MATLAB script to automatically count the number of H2BGFP-positive cones in the central half of the retina, since $\mathrm{RP}$ cones degenerate faster in the central than the peripheral retina. The algorithm was based on a Gaussian model to identify the centers of labeled cells, and published recently (Wu et al., n.d.). The threshold of peak intensity and the variance of distribution were initially determined using visual inspection, and a comparison to the number of manually counted cones from 6 retinas. The threshold of intensity and variance thus determined were then set at fixed values for all the experiments that used cone quantification. The background intensity did not interfere with the accurate counting on the raw images by this MATLAB script, despite the representative images at low-magnification might look differently.

\section{Live-imaging of cones on ex vivo retinal explants}

For JC-1 mitochondrial dye staining, the retina was quickly dissected in a solution of $50 \%$ Ham's F12 Nutrient Mix (11765054, Thermo Fisher Scientific) and 50\% Dulbecco's Modified Eagle Medium (DMEM; 11995065, Thermo Fisher Scientific) at room temperature. They were then incubated in a culture solution containing 50\% Fluorobrite DMEM (A1896701, Thermo Fisher Scientific), 25\% heat inactivated horse serum (26050088, Thermo Fisher Scientific) and 25\% Hanks' Balanced Salt Solution (HBSS; 14065056, Thermo Fisher Scientific) with $2 \mu \mathrm{M}$ JC-1 dye (M34152, Thermo Fisher Scientific) at $37^{\circ} \mathrm{C}$ in a $5 \% \mathrm{CO}_{2}$ incubator for 20 minutes. The retinas were washed in $37^{\circ} \mathrm{C}$ culture medium without $\mathrm{JC}-1$ for three times, transferred in a glass-bottom culture dish (MatTek P50G-1.5-30-F) with culture medium, and imaged using a confocal microscope (LSM710 Zeiss), which was equipped with a chamber pre-heated to $37^{\circ} \mathrm{C}$ with pre-filled $5 \% \mathrm{CO}_{2}$. Right before imaging, a cover slip (VWR 89015-725) was gently applied to flatten the retina. Regions of interest (with H2BGFP as an indicator of successful AAV infection and to set the correct focal plane on the 
cone layer) were selected under the eyepiece with a 63x objective (Plan Apo 63X/1.4 Oil DIC III). Fluorescent images from the same region of interest were obtained with the excitation-wavelength in the order of $561 \mathrm{~nm}$ (for J-aggregates), $514 \mathrm{~nm}$ (for JC-1 monomer), and $488 \mathrm{~nm}$ (for H2BGFP). Four different regions of interest from the central part of the same retina were imaged before moving to the next retina.

For $\mathrm{RH} 421\left(\mathrm{Na}^{+} / \mathrm{K}^{+}\right.$ATPase dye) staining, similar steps were taken as for JC-1 staining, with the following modifications: 1) $0.83 \mu \mathrm{M} \mathrm{RH} 421$ dye (61017, Biotium) was added to the glass-bottom culture dishes just before imaging, but not during incubation in the incubator, due to the fast action of $\mathrm{RH} 421.2) 5$ regions of interest were imaged per retina from the central area. 3) The dissection and culture medium were lactate-only medium (see below). 4) Excitation wavelengths: $561 \mathrm{~nm}$ (RH421), and $488 \mathrm{~nm}$ (H2BGFP).

For imaging genetically-encoded metabolic sensors (PercevalHR, iGlucoSnFR and pHRed), retinas were placed in the incubator for 12 minutes and then taken to confocal imaging without any staining. For the high-glucose condition, the culture medium described above contains $\approx 15 \mathrm{mM}$ glucose without lactate or pyruvate. For the lactate-only condition, the culture and dissection media were both glucose-pyruvate-free DMEM (A144300, Thermo Fisher Scientific) and were supplemented with $20 \mathrm{mM}$ sodium L-lactate (71718, Sigma-Aldrich). For the pyruvate-only condition, the culture and dissection media, were both glucose-pyruvate-free DMEM plus 10 or 20 mM sodium pyruvate (P2256, Sigma-Aldrich). No AAV-H2BGFP was co-injected with these sensors, since the sensors themselves could be used to trace the area of infection. The excitation wavelengths for sensors were $488 \mathrm{~nm}$ and $405 \mathrm{~nm}$ (PercevalHR, ratiometric high and low ATP:ADP), $488 \mathrm{~nm}$ and $561 \mathrm{~nm}$ (iGlucoSnFR, glucose-sensing GFP and normalization mRuby), and $561 \mathrm{~nm}$ and $458 \mathrm{~nm}$ (pHRed, ratiometric low and high $\mathrm{pH}$ ).

The fluorescent intensity of all acquired images was measured by ImageJ. The ratio of sensors/dye was normalized to averaged control results taken at the same condition.

\section{Flow cytometry and cell sorting}

All flow cytometry and cell sorting were performed on MoFlo Astrios EQ equipment. Retinas were freshly dissected and dissociated using cysteine-activated papain followed by gentle pipetting (Shekhar et al., 2016). Before sorting, all samples were passed through a 35- $\mu \mathrm{m}$ filter with buffer containing Fluorobrite DMEM (A1896701, Thermo Fisher Scientific) and 0.4\% BSA. Cones labeled with AAV8-SynP136-H2BGFP (highly cone-specific) were sorted into the appropriate buffer for either ddPCR or RNA-sequencing.

\section{RNA sequencing}

RNA sequencing was done as previously described (Wang et al., 2019). 1,000 H2BGFP-positive cones per retina were sorted into $10 \mu \mathrm{L}$ of Buffer TCL (Qiagen) containing $1 \% \beta$-mercaptoethanol and immediately frozen in $-80^{\circ} \mathrm{C}$. On the day of sample submission, the frozen cone lysates were thawed on ice and loaded into a 96-well plate for cDNA library synthesis and sequencing. A modified Smart-Seq2 protocol was performed on samples by the Broad Institute Genomics Platform with $\sim 6$ million reads per sample (Picelli et al., 2013). The reads were mapped to the GRCm38.p6 reference genome after quality control measures. Reads assigned to each gene were quantified using featureCounts (Liao et al., 2014). Count data were analyzed using DESeq2 to identify differentially expressed genes, with an adjusted $p$ value less than 0.05 considered significant(Anders and Huber, 2010). The raw results have been deposited to Gene Expression Omnibus (accession number GSE161622). 
$\underline{\text { ddPCR }}$

RNA was isolated from 20,000 sorted cones per retina using RNeasy Micro Kit (Qiagen) as previously described (Wang et al., 2020), and converted to cDNA using the SuperScript III FirstStrand Synthesis System (Invitrogen). cDNA from each sample was packaged in droplets for Droplet Digital $^{\mathrm{TM}}$ PCR (ddPCR) using QX200 EvaGreen Supermix (\#1864034). The reads of expression were normalized to the housekeeping gene Hprt. Sequences for RT-PCR primers were designed using the IDT online RealTime qPCR primer design tool. The following primers were selected for the genes of interest: Txnip (forward 5'-ACATTATCTCAGGGACTTGCG-3'; reverse 5'-AAGGATGACTTTCTTGGAGCC-3'), Hprt (forward 5'-TCAGTCAACGGGGGACATAAA-3'; reverse 5'-GGGGCTGTACTGCTTAACCAG-3'), mt-Nd4 (forward 5'-

AGCTCAATCTGCTTACGCCA-3'; reverse 5'-TGTGAGGCCATGTGCGATTA-3'), mt-Cytb (forward 5'-ATTCTACGCTCAATCCCCAAT-3'; reverse 5'-TATGAGATGGAGGCTAGTTGGC-3'), mt-Co1 (forward 5'-TCTGTTCTGATTCTTTGGGCACC-3'; reverse 5'-CTACTGTGAATATGTGGTGGGCT3', Acs/3 (forward 5'- AACCACGTATCTTCAACACCATC-3'; reverse 5'AGTCCGGTTTGGAACTGACAG-3'), and Ftl1 (forward 5'- CCATCTGACCAACCTCCGC-3'; reverse 5'- CGCTCAAAGAGATACTCGCC-3')).

\section{Electron microscopy}

Intracardial perfusion (4\% PFA+1\% glutaraldehyde) was performed on ketamine/xylazine (100/10 $\mathrm{mg} / \mathrm{kg}$ ) anesthetized mice before the removal of eyes. The cornea was sliced open and the eye was fixed with a fixative buffer $(1.25 \%$ formaldehyde $+2.5 \%$ glutaraldehyde $+0.03 \%$ picric acid in $0.1 \mathrm{M}$ sodium cacodylate buffer, $\mathrm{pH} 7.4$ ) overnight at $4^{\circ} \mathrm{C}$. The cornea, lens and retina were removed before resin embedding, ultrathin sectioning and negative staining at Harvard Medical School Electron Microscopy Core. The detailed methods can be found on the core's website (https://electron-microscopy.hms.harvard.edu/methods). The stained thin sections were imaged on a conventional transmission electron microscope (JEOL 1200EX) with an AMT 2k CCD camera.

\section{Statistics}

For the comparison of two sample groups, two-tailed unpaired Student's t test was used to test for the significance of difference, except for P140 rho-/- optomotor assay (paired two-tail t-test). For comparison of more than two sample groups, ANOVA and Dunnett's multiple comparison test was performed in Prism 8 software to determine the significance. A $p$ value of less than 0.05 was considered statistically significant. All error bars are presented as mean \pm standard deviation, except for the rd10 optomotor assays (mean \pm SEM).

\section{Study approval}

All animal experiments were approved by the IACUC of Harvard University in accordance with institutional guidelines. 


\section{References}

Aït-Ali N, Fridlich R, Millet-Puel G, Clérin E, Delalande F, Jaillard C, Blond F, Perrocheau L, Reichman S, Byrne LCC, Olivier-Bandini A, Bellalou J, Moyse E, Bouillaud F, Nicol X, Dalkara D, van Dorsselaer A, Sahel J-A, Léveillard T, van Dorsselaer A, Sahel J-A, Léveillard T. 2015. Rod-Derived Cone Viability Factor Promotes Cone Survival by Stimulating Aerobic Glycolysis. Cell 161:817-832. doi:10.1016/j.cell.2015.03.023

Anders S, Huber W. 2010. Differential expression analysis for sequence count data. Genome Biol 11:R106. doi:10.1186/gb-2010-11-10-r106

Brodier L, Rodrigues T, Matter-Sadzinski L, Matter J-M. 2020. A transient decrease in mitochondrial activity is required to establish the ganglion cell fate in retina adapted for high acuity vision. bioRxiv 2020.03.23.002998. doi:10.1101/2020.03.23.002998

Byrne LC, Dalkara D, Luna G, Fisher SK, Clérin E, Sahel J-A, Léveillard T, Flannery JG. 2015. Viral-mediated RdCVF and RdCVFL expression protects cone and rod photoreceptors in retinal degeneration. J Clin Invest 125:105-16. doi:10.1172/JCI65654

Cehajic-Kapetanovic J, Xue K, Martinez-Fernandez de la Camara C, Nanda A, Davies A, Wood LJ, Salvetti AP, Fischer MD, Aylward JW, Barnard AR, Jolly JK, Luo E, Lujan BJ, Ong T, Girach A, Black GCM, Gregori NZ, Davis JL, Rosa PR, Lotery AJ, Lam BL, Stanga PE, MacLaren RE. 2020. Initial results from a first-in-human gene therapy trial on X-linked retinitis pigmentosa caused by mutations in RPGR. Nat Med. doi:10.1038/s41591-020-0763-1

Chang B, Hawes NL, Pardue MT, German AM, Hurd RE, Davisson MT, Nusinowitz S, Rengarajan K, Boyd AP, Sidney SS, Phillips MJ, Stewart RE, Chaudhury R, Nickerson JM, Heckenlively JR, Boatright JH. 2007. Two mouse retinal degenerations caused by missense mutations in the $\beta$-subunit of rod cGMP phosphodiesterase gene. Vision Res 47:624-633. doi:10.1016/j.visres.2006.11.020

Chinchore Y, Begaj T, Guillermeir C, Steinhauser M, Punzo C, Cepko C. 2019. Transduction of gluconeogenic enzymes prolongs cone photoreceptor survival and function in models of retinitis pigmentosa. bioRxiv 569665. doi:10.1101/569665

Chinchore Y, Begaj T, Wu D, Drokhlyansky E, Cepko CL. 2017. Glycolytic reliance promotes anabolism in photoreceptors. Elife 6. doi:10.7554/eLife.25946

DeBalsi KL, Wong KE, Koves TR, Slentz DH, Seiler SE, Wittmann AH, Ilkayeva OR, Stevens RD, Perry CGR, Lark DS, Hui ST, Szweda L, Neufer PD, Muoio DM. 2014. Targeted Metabolomics Connects Thioredoxin-interacting Protein (TXNIP) to Mitochondrial Fuel Selection and Regulation of Specific Oxidoreductase Enzymes in Skeletal Muscle. J Biol Chem 289:8106-8120. doi:10.1074/jbc.M113.511535

Esumi N, Kachi S, Hackler L, Masuda T, Yang Z, Campochiaro PA, Zack DJ. 2009. BEST1 expression in the retinal pigment epithelium is modulated by OTX family members. Hum Mol Genet 18:128-141. doi:10.1093/hmg/ddn323

Eventoff W, Rossmann MG, Taylor SS, Torff HJ, Meyer H, Keil W, Kiltz HH. 1977. Structural adaptations of lactate dehydrogenase isozymes. Proc Natl Acad Sci U S A 74:2677-2681. doi:10.1073/pnas.74.7.2677

Fedosova NU, Cornelius F, Klodos I. 1995. Fluorescent Styryl Dyes as Probes for Na,K-ATPase Reaction Mechanism: Significance of the Charge of the Hydrophilic Moiety of RH Dyes. Biochemistry 34:16806-16814. doi:10.1021/bi00051a031

Fehr AR, Singh SA, Kerr CM, Mukai S, Higashi H, Aikawa M. 2020. The impact of PARPs and ADP-ribosylation on inflammation and host-pathogen interactions. Genes Dev. doi:10.1101/gad.334425.119

Ferrando RE, Newton K, Chu F, Webster JD, French DM. 2015. Immunohistochemical Detection of FLAG-Tagged Endogenous Proteins in Knock-In Mice. J Histochem Cytochem 63:244-255. doi:10.1369/0022155414568101

Forred BJ, Neuharth S, Kim DI, Amolins MW, Motamedchaboki K, Roux KJ, Vitiello PF. 2016. Identification of Redox and Glucose-Dependent Txnip Protein Interactions. Oxid Med Cell Longev 2016. doi:10.1155/2016/5829063 
734 Giering JC, Grimm D, Storm TA, Kay MA. 2008. Expression of shRNA from a tissue-specific pol II promoter is an effective and safe RNAi therapeutic. Mol Ther 16:1630-1636.

doi:10.1038/mt.2008.144

Gospe SM, Baker SA, Arshavsky VY. 2010. Facilitative glucose transporter Glut1 is actively excluded from rod outer segments. J Cell Sci 123:3639-3644. doi:10.1242/jcs.072389

Grenell A, Wang Y, Yam M, Swarup A, Dilan TL, Hauer A, Linton JD, Philp NJ, Gregor E, Zhu S, Shi Q, Murphy J, Guan T, Lohner D, Kolandaivelu S, Ramamurthy V, Goldberg AFX, Hurley JB, Du J. 2019. Loss of MPC1 reprograms retinal metabolism to impair visual function. Proc Natl Acad Sci U S A 116:3530-3535. doi:10.1073/pnas.1812941116

Grieger JC, Choi VW, Samulski RJ. 2006. Production and characterization of adeno-associated viral vectors. Nat Protoc. doi:10.1038/nprot.2006.207

Hartong DT, Berson EL, Dryja TP. 2006. Retinitis pigmentosa. Lancet 368:1795-809. doi:10.1016/S0140-6736(06)69740-7

Hoang Q, Linsenmeier R, Chung C, Curcio C. 2002. Photoreceptor inner segments in monkey and human retina: mitochondrial density, optics, and regional variation. Vis Neurosci 19:395-407. doi:10.1017/s0952523802194028

Hocsak E, Szabo V, Kalman N, Antus C, Cseh A, Sumegi K, Eros K, Hegedus Z, Gallyas F, Sumegi B, Racz B. 2017. PARP inhibition protects mitochondria and reduces ROS production via PARP-1-ATF4-MKP-1-MAPK retrograde pathway. Free Radic Biol Med 108:770-784. doi:10.1016/j.freeradbiomed.2017.04.018

Hood DA, Adhihetty PJ, Colavecchia M, Gordon JW, Irrcher I, Joseph AM, Lowe ST, Rungi AA. 2003. Mitochondrial biogenesis and the role of the protein import pathway. Med Sci Sports Exerc 35:86-94. doi:10.1097/00005768-200301000-00015

Hui S, Ghergurovich JM, Morscher RJ, Jang C, Teng X, Lu W, Esparza LA, Reya T, Zhan L, Yanxiang Guo J, White E, Rabinowitz JD. 2017. Glucose feeds the TCA cycle via circulating lactate. Nature 551:115-118. doi:10.1038/nature24057

Hwang J, Suh HW, Jeon YH o., Hwang E, Nguyen LT, Yeom J, Lee SG, Lee C, Kim KJ i., Kang BS i., Jeong JO, Oh TK, Choi I, Lee JO, Kim MH e. 2014. The structural basis for the negative regulation of thioredoxin by thioredoxin-interacting protein. Nat Commun 5:2958.

doi:10.1038/ncomms3958

Ingram NT, Fain GL, Sampath AP. 2020. Elevated energy requirement of cone photoreceptors. Proc Natl Acad Sci U S A 117:19599-19603. doi:10.1073/pnas.2001776117

Jiang BH, Rue E, Wang GL, Roe R, Semenza GL. 1996. Dimerization, DNA binding, and transactivation properties of hypoxia- inducible factor 1. J Biol Chem 271:17771-17778. doi:10.1074/jbc.271.30.17771

Joyal J-S, Sun Y, Gantner ML, Shao Z, Evans LP, Saba N, Fredrick T, Burnim S, Kim JS, Patel G, Juan AM, Hurst CG, Hatton CJ, Cui Z, Pierce KA, Bherer P, Aguilar E, Powner MB, Vevis K, Boisvert M, Fu Z, Levy E, Fruttiger M, Packard A, Rezende FA, Maranda B, Sapieha P, Chen J, Friedlander M, Clish CB, Smith LEH. 2016. Retinal lipid and glucose metabolism dictates angiogenesis through the lipid sensor Ffar1. Nat Med 22:439-445. doi:10.1038/nm.4059 Junn E, Han SH, Im JY, Yang Y, Cho EW, Um HD, Kim DK, Lee KW, Han PL, Rhee SG, Choi I. 2000. Vitamin D3 up-regulated protein 1 mediates oxidative stress via suppressing the thioredoxin function. J Immunol 164:6287-95.

Jüttner J, Szabo A, Gross-Scherf B, Morikawa RK, Rompani SB, Hantz P, Szikra T, Esposti F, Cowan CS, Bharioke A, Patino-Alvarez CP, Keles Ö, Kusnyerik A, Azoulay T, HartI D, Krebs AR, Schübeler D, Hajdu RI, Lukats A, Nemeth J, Nagy ZZ, Wu KC, Wu RH, Xiang L, Fang XL, Jin ZB, Goldblum D, Hasler PW, Scholl HPN, Krol J, Roska B. 2019. Targeting neuronal and glial cell types with synthetic promoter AAVs in mice, non-human primates and humans. Nat Neurosci 22:1345-1356. doi:10.1038/s41593-019-0431-2

Kang Y, Zhou XE, Gao X, He Y, Liu W, Ishchenko A, Barty A, White TA, Yefanov O, Han GW, Xu Q, De Waal PW, Ke J, Tan MHE, Zhang C, Moeller A, West GM, Pascal BD, Van Eps N, Caro LN, Vishnivetskiy SA, Lee RJ, Suino-Powell KM, Gu X, Pal K, Ma J, Zhi X, Boutet S, Williams 
bioRxiv preprint doi: https://doi.org/10.1101/2021.01.27.428411; this version posted January $27,2021$. The copyright holder for this preprint

(which was not certified by peer review) is the author/funder, who has granted bioRxiv a license to display the preprint in perpetuity. It is made available under aCC-BY 4.0 International license.

GJ, Messerschmidt M, Gati C, Zatsepin NA, Wang D, James D, Basu S, Roy-Chowdhury S, Conrad CE, Coe J, Liu H, Lisova S, Kupitz C, Grotjohann I, Fromme R, Jiang Y, Tan M, Yang H, Li J, Wang M, Zheng Z, Li D, Howe N, Zhao Y, Standfuss J, Diederichs K, Dong Y, Potter $\mathrm{CS}$, Carragher B, Caffrey M, Jiang H, Chapman HN, Spence JCH, Fromme P, Weierstall U, Ernst OP, Katritch V, Gurevich V V., Griffin PR, Hubbell WL, Stevens RC, Cherezov V, Melcher K, Xu HE. 2015. Crystal structure of rhodopsin bound to arrestin by femtosecond Xray laser. Nature 523:561-567. doi:10.1038/nature14656

Kanow MA, Giarmarco MM, Jankowski CS, Tsantilas K, Engel AL, Du J, Linton JD, Farnsworth CC, Sloat SR, Rountree A, Sweet IR, Lindsay KJ, Parker ED, Brockerhoff SE, Sadilek M, Chao JR, Hurley JB. 2017. Biochemical adaptations of the retina and retinal pigment epithelium support a metabolic ecosystem in the vertebrate eye. Elife $\mathbf{6}$. doi:10.7554/eLife.28899

Katsu-Jiménez $Y$, Vázquez-Calvo $C$, Maffezzini C, Halldin M, Peng X, Freyer C, Wredenberg A, Giménez-Cassina A, Wedell A, Arnér ESJ. 2019. Absence of TXNIP in humans leads to lactic acidosis and low serum methionine linked to deficient respiration on pyruvate. Diabetes 68:709-723. doi:10.2337/db18-0557

Keller J, Marvin J, Lacin H, Lemon W, Shea J, Kim S, Lee R, Koyama M, Keller P, Looger L. 2019. In Vivo Glucose Imaging in Multiple Model Organisms with an Engineered Single-Wavelength Sensor. SSRN Electron J 571422. doi:10.1101/571422

Komeima K, Rogers BS, Lu L, Campochiaro PA. 2006. Antioxidants reduce cone cell death in a model of retinitis pigmentosa. Proc Natl Acad Sci U S A 103:11300-11305. doi:10.1073/pnas.0604056103

Lem J, Krasnoperova N V., Calvert PD, Kosaras B, Cameron DA, Nicolò M, Makino CL, Sidman RL. 1999. Morphological, physiological, and biochemical changes in rhodopsin knockout mice. Proc Natl Acad Sci U S A 96:736-741. doi:10.1073/pnas.96.2.736

Liao Y, Smyth GK, Shi W. 2014. FeatureCounts: An efficient general purpose program for assigning sequence reads to genomic features. Bioinformatics 30:923-930. doi:10.1093/bioinformatics/btt656

Maguire AM, Russell S, Wellman JA, Chung DC, Yu ZF, Tillman A, Wittes J, Pappas J, Elci O, Marshall KA, McCague S, Reichert H, Davis M, Simonelli F, Leroy BP, Wright JF, High KA, Bennett J. 2019. Efficacy, Safety, and Durability of Voretigene Neparvovec-rzyl in RPE65 Mutation-Associated Inherited Retinal Dystrophy: Results of Phase 1 and 3 Trials. Ophthalmology 126:1273-1285. doi:10.1016/j.ophtha.2019.06.017

Majmundar AJ, Wong WJ, Simon MC. 2010. Hypoxia-inducible factors and the response to hypoxic stress. Mol Cell 40:294-309. doi:10.1016/j.molcel.2010.09.022

Matsuda T, Cepko CL. 2007. Controlled expression of transgenes introduced by in vivo electroporation. Proc Natl Acad Sci 104:1027-1032. doi:10.1073/pnas.0610155104

Nishinaka Y, Masutani H, Nakamura H, Yodoi J. 2001. Regulatory roles of thioredoxin in oxidative stress-induced cellular responses. Redox Rep. doi:10.1179/135100001101536427

Nishiyama A, Matsui M, Iwata S, Hirota K, Masutani H, Nakamura H, Takagi Y, Sono H, Gon Y, Yodoi J. 1999. Identification of thioredoxin-binding protein-2/vitamin D(3) up-regulated protein 1 as a negative regulator of thioredoxin function and expression. J Biol Chem 274:21645-50.

Okawa H, Sampath AP, Laughlin SB, Fain GL. 2008. ATP consumption by mammalian rod photoreceptors in darkness and in light. Curr Biol 18:1917-21. doi:10.1016/j.cub.2008.10.029

Patwari P, Chutkow WA, Cummings K, Verstraeten VLRM, Lammerding J, Schreiter ER, Lee RT. 2009. Thioredoxin-independent regulation of metabolism by the $\alpha$-arrestin proteins. J Biol Chem 284:24996-25003. doi:10.1074/jbc.M109.018093

Picelli S, Björklund ÅK, Faridani OR, Sagasser S, Winberg G, Sandberg R. 2013. Smart-seq2 for sensitive full-length transcriptome profiling in single cells. Nat Methods 10:1096-1098. doi:10.1038/nmeth.2639

Prusky GT, Alam NM, Beekman S, Douglas RM. 2004. Rapid quantification of adult and developing mouse spatial vision using a virtual optomotor system. Investig Ophthalmol Vis Sci 
bioRxiv preprint doi: https://doi.org/10.1101/2021.01.27.428411; this version posted January 27, 2021. The copyright holder for this preprint

(which was not certified by peer review) is the author/funder, who has granted bioRxiv a license to display the preprint in perpetuity. It is made available under aCC-BY 4.0 International license.

\section{5:4611-4616. doi:10.1167/iovs.04-0541}

Puca L, Brou C. 2014. a-Arrestins - new players in Notch and GPCR signaling pathways in mammals. J Cell Sci. doi:10.1242/jcs.142539

Punzo C, Kornacker K, Cepko CL. 2009. Stimulation of the insulin/mTOR pathway delays cone death in a mouse model of retinitis pigmentosa. Nat Neurosci 12:44-52. doi:10.1038/nn.2234

Punzo C, Xiong W, Cepko CL. 2012. Loss of daylight vision in retinal degeneration: are oxidative stress and metabolic dysregulation to blame? J Biol Chem 287:1642-8. doi:10.1074/jbc.R111.304428

Reers M, Smiley ST, Mottola-Hartshorn C, Chen A, Lin M, Chen LB. 1995. Mitochondrial membrane potential monitored by JC-1 dye. Methods Enzymol 260:406-17.

Robinson C, Stringer S. 2001. The splice variants of vascular endothelial growth factor (VEGF) and their receptors - PubMed. J Cell Sci 114:853-65.

Shekhar K, Lapan SW, Whitney IE, Tran NM, Macosko EZ, Kowalczyk M, Adiconis X, Levin JZ, Nemesh J, Goldman M, McCarroll SA, Cepko CL, Regev A, Sanes JR. 2016. Comprehensive Classification of Retinal Bipolar Neurons by Single-Cell Transcriptomics. Cell 166:13081323.e30. doi:10.1016/j.cell.2016.07.054

Shriver LP, Manchester M. 2011. Inhibition of fatty acid metabolism ameliorates disease activity in an animal model of multiple sclerosis. Sci Rep 1:79. doi:10.1038/srep00079

Swarup A, Samuels IS, Bell BA, Han JYS, Du J, Massenzio E, Abel ED, Boesze-Battaglia K, Peachey NS, Philp NJ. 2019. Modulating GLUT1 expression in retinal pigment epithelium decreases glucose levels in the retina: Impact on photoreceptors and müller glial cells. Am J Physiol - Cell Physio/ 316:C121-C133. doi:10.1152/ajpcell.00410.2018

Szczesny B, Brunyanszki A, Olah G, Mitra S, Szabo C. 2014. Opposing roles of mitochondrial and nuclear PARP1 in the regulation of mitochondrial and nuclear DNA integrity: Implications for the regulation of mitochondrial function. Nucleic Acids Res 42:13161-13173. doi:10.1093/nar/gku1089

Tantama M, Hung YP, Yellen G. 2011. Imaging intracellular pH in live cells with a genetically encoded red fluorescent protein sensor. J Am Chem Soc 133:10034-7. doi:10.1021/ja202902d

Tantama M, Martínez-François JR, Mongeon R, Yellen G. 2013. Imaging energy status in live cells with a fluorescent biosensor of the intracellular ATP-to-ADP ratio. Nat Commun 4:2550. doi: $10.1038 /$ ncomms 3550

Waldhart AN, Dykstra H, Peck AS, Boguslawski EA, Madaj ZB, Wen J, Veldkamp K, Hollowell M, Zheng B, Cantley LC, McGraw TE, Wu N. 2017. Phosphorylation of TXNIP by AKT Mediates Acute Influx of Glucose in Response to Insulin. Cell Rep 19:2005-2013. doi:10.1016/j.celrep.2017.05.041

Wang S, Sengel C, Emerson MM, Cepko CL. 2014. A Gene Regulatory Network Controls the Binary Fate Decision of Rod and Bipolar Cells in the Vertebrate Retina. Dev Cell 30:513-527. doi:10.1016/j.devcel.2014.07.018

Wang SK, Xue Y, Cepko CL. 2020. Microglia modulation by TGF- $\beta 1$ protects cones in mouse models of retinal degeneration. J Clin Invest 140:4360-4369. doi:10.1172/JCI136160

Wang SK, Xue Y, Rana P, Hong CM, Cepko CL. 2019. Soluble CX3CL1 gene therapy improves cone survival and function in mouse models of retinitis pigmentosa. Proc Natl Acad Sci U S A 116:10140-10149. doi:10.1073/pnas.1901787116

Wang W, Lee SJ, Scott PA, Lu X, Emery D, Liu Y, Ezashi T, Roberts MR, Ross JW, Kaplan HJ, Dean DC. 2016. Two-Step Reactivation of Dormant Cones in Retinitis Pigmentosa. Cell Rep 15:372-385. doi:10.1016/j.celrep.2016.03.022

Warburg O. 1925. The metabolism of carcinoma cells. J Cancer Res 9:148-163. doi:10.1158/jcr.1925.148

Wellard J, Lee D, Valter K, Stone J. 2005. Photoreceptors in the rat retina are specifically vulnerable to both hypoxia and hyperoxia. Vis Neurosci 22:501-507. doi:10.1017/S0952523805224112 
bioRxiv preprint doi: https://doi.org/10.1101/2021.01.27.428411; this version posted January 27, 2021. The copyright holder for this preprint (which was not certified by peer review) is the author/funder, who has granted bioRxiv a license to display the preprint in perpetuity. It is made available under aCC-BY 4.0 International license.

Wu D, Ji X, Ivanchenko M, Chung M, Piper M, Wang S, Xue Y, Xu H, West E, Zhao S, Xiong W, Cepko C. n.d. Nrf2 overexpression rescues the RPE in mouse models of retinitis pigmentosa. JCl Insight.

Wu N, Zheng B, Shaywitz A, Dagon Y, Tower C, Bellinger G, Shen C-H, Wen J, Asara J, McGraw TE, Kahn BB, Cantley LC. 2013. AMPK-dependent degradation of TXNIP upon energy stress leads to enhanced glucose uptake via GLUT1. Mol Cell 49:1167-75. doi:10.1016/j.molcel.2013.01.035

Xiong W, MacColl Garfinkel AE, Li Y, Benowitz LI, Cepko CL. 2015. NRF2 promotes neuronal survival in neurodegeneration and acute nerve damage. J Clin Invest 125:1433-45. doi:10.1172/JCI79735

Xiong W, Wu DM, Xue Y, Wang SK, Chung MJ, Ji X, Rana P, Zhao SR, Mai S, Cepko CL. 2019. AAV cis-regulatory sequences are correlated with ocular toxicity. Proc Natl Acad Sci U S A 116:5785-5794. doi:10.1073/pnas.1821000116

Ye GJ, Budzynski E, Sonnentag P, Nork TM, Sheibani N, Gurel Z, Boye SL, Peterson JJ, Boye SE, Hauswirth WW, Chulay JD. 2016. Cone-Specific Promoters for Gene Therapy of Achromatopsia and Other Retinal Diseases. Hum Gene Ther 27:72-82. doi:10.1089/hum.2015.130

Zhang S, Xie C. 2017. The role of OXCT1 in the pathogenesis of cancer as a rate-limiting enzyme of ketone body metabolism. Life Sci 183:110-115. doi:10.1016/j.Ifs.2017.07.003

Zhao L, Zabel MK, Wang X, Ma W, Shah P, Fariss RN, Qian H, Parkhurst CN, Gan W, Wong WT. 2015. Microglial phagocytosis of living photoreceptors contributes to inherited retinal degeneration. EMBO Mol Med 7:1179-1197. doi:10.15252/emmm.201505298 


\section{Acknowledgements}

715 We thank Sui Wang, ChangHee Lee, Sylvain Lapan, Gabby Niconchuk, Brian Rabe, Cem Sengel, $\exists 16$ Sophia Zhao, Yuji Atsuta, Wenjun Xiong, Ryoji Amamoto, Grace Wallick, Gary Yellen, Zhongjie э17 Fu, Zhengping Hu, Maryna Ivanchenko, Paula Montero-Llopis, Microscopy Resources on the 318 North Quad, Maria Ericsson, Electron Microscopy Facility, Flow Cytometry of Immunology, 719 Marcelo Cicconet, Image and Data Analysis Core of Harvard Medical School, Genomics Platform

\section{Author contributions}

Y.X. and C.L.C. designed the study and wrote the manuscript with input from other authors. Y.X., S.K.W. and P.R. performed the experiments. Y.X., S.K.W., P.R., E.R.W., C.M.H. and H.F. analyzed the data. D.M.W provided critical software and reagents to this study. 
$\exists 40$ Fig. 1: Txnip enhances cone survival and delays the deterioration of cone-mediated vision $\exists 41$ in RP mice.

$\exists 42$ a. Representative images from $\mathrm{P} 20$ and $\mathrm{P} 50 \mathrm{rd} 1, \mathrm{P} 130 \mathrm{rd} 10$ and $\mathrm{P} 150 \mathrm{rho}^{-/-}$flat-mounted retinas, in which cones are labeled with H2BGFP, treated with Txnip or control (i.e. H2BGFP and vehicle only, same applies to all other figures). The outer circle was drawn to mark the full extent of the retina, and the inner circle was drawn by using half of the radius of the outer circle. The small box in the top four panels are zoomed in with pixels recognized as cones by a MATLAB automatedcounting program (Extended Data Fig.1c). The number at lower right corner is the count of cells within the half radius of each image (same applies to all other figures). 
a

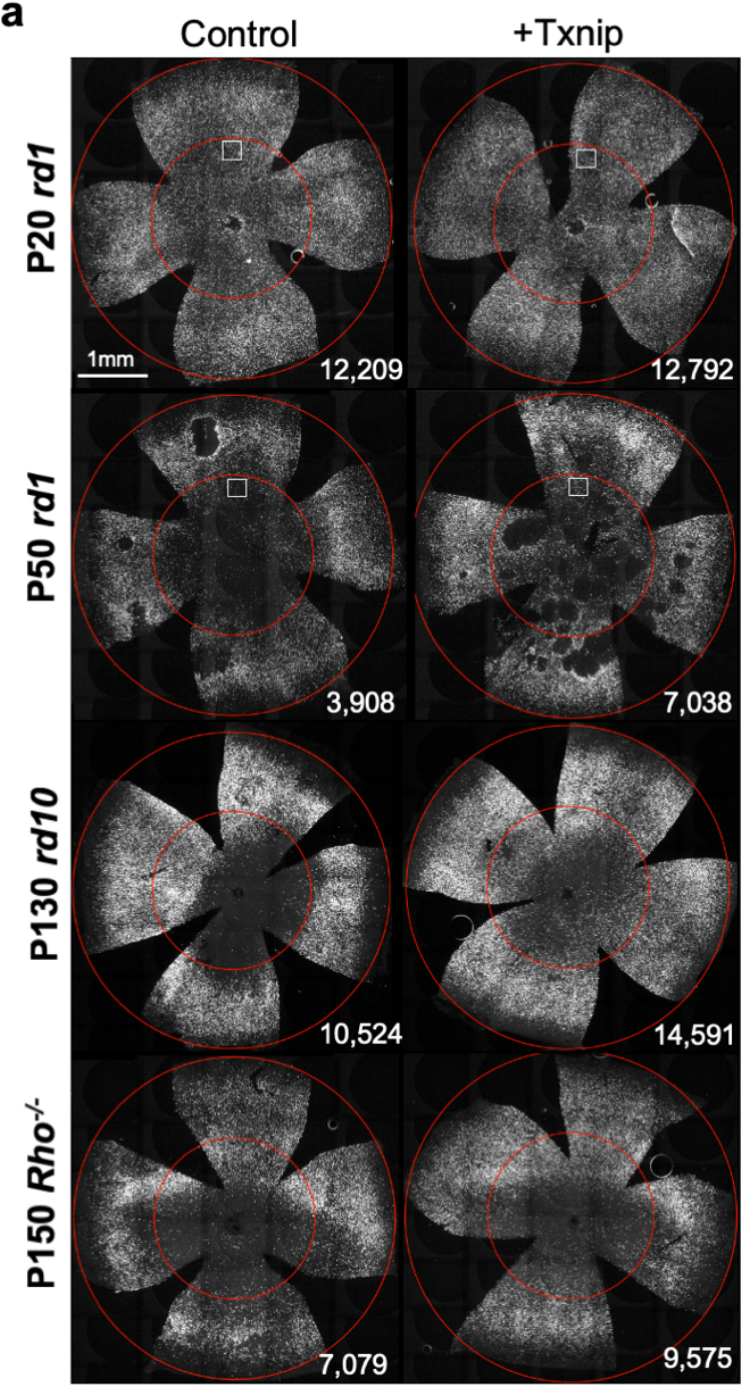

b
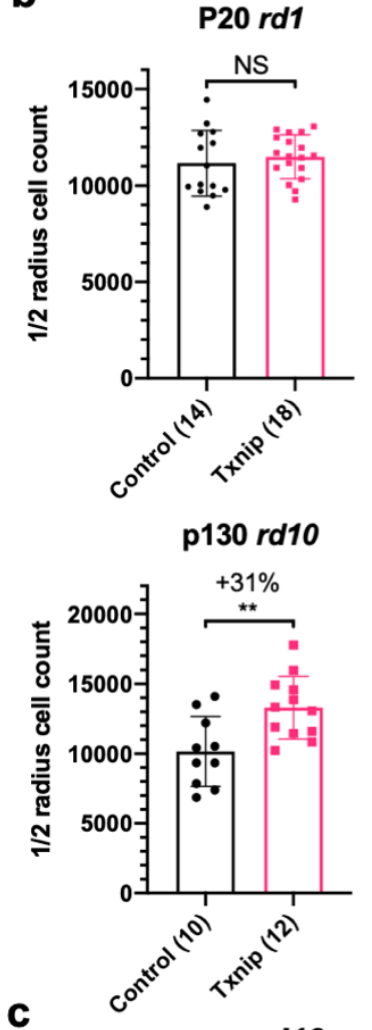

C $\quad r d 10$

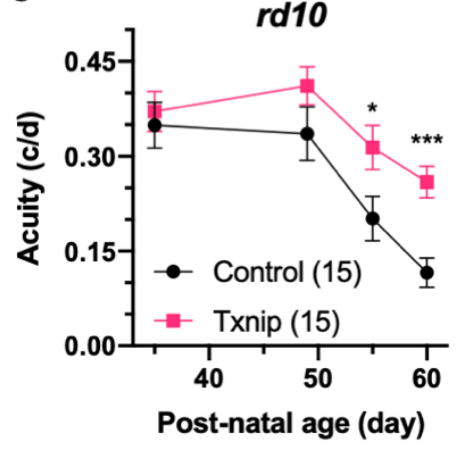

P50 rd1

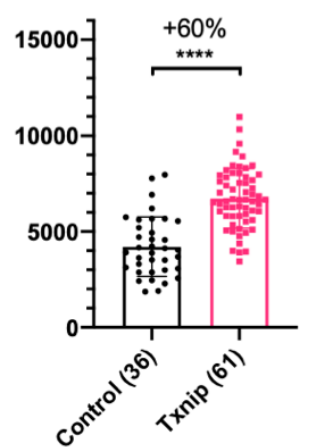

P150 rho-

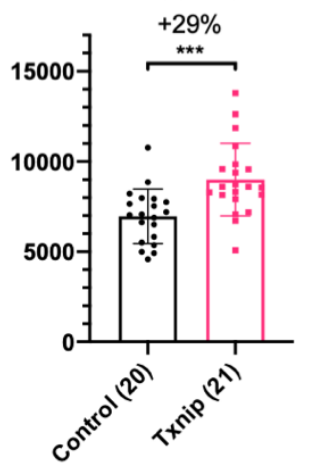

P140 rho $\%$

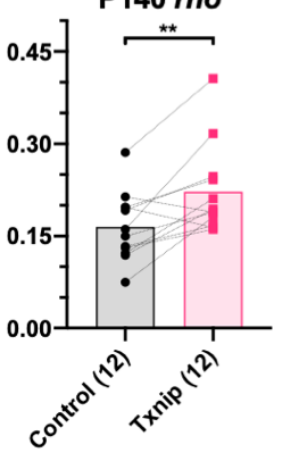




\section{Fig. 2: Test of Txnip alleles on cone survival}

761 a. Representative P50 rd1 flat-mounted retinas with H2BGFP (gray) labeled cones treated with one of four different Txnip alleles.

364 wildtype (wt) Txnip, Txnip alleles, and control. Error bar: standard deviation. Abbreviations:

365 Txnip.CS.SA = Txnip.C247S.S308A; Txnip.CS.LLAA = Txnip.C247S.LL351\&352AA.

366

767 


\section{a}
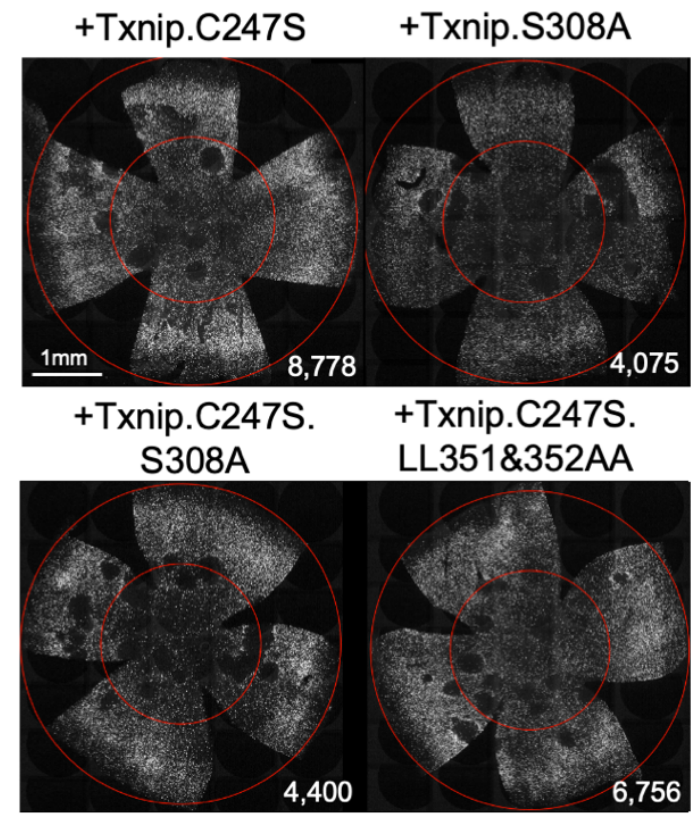

b

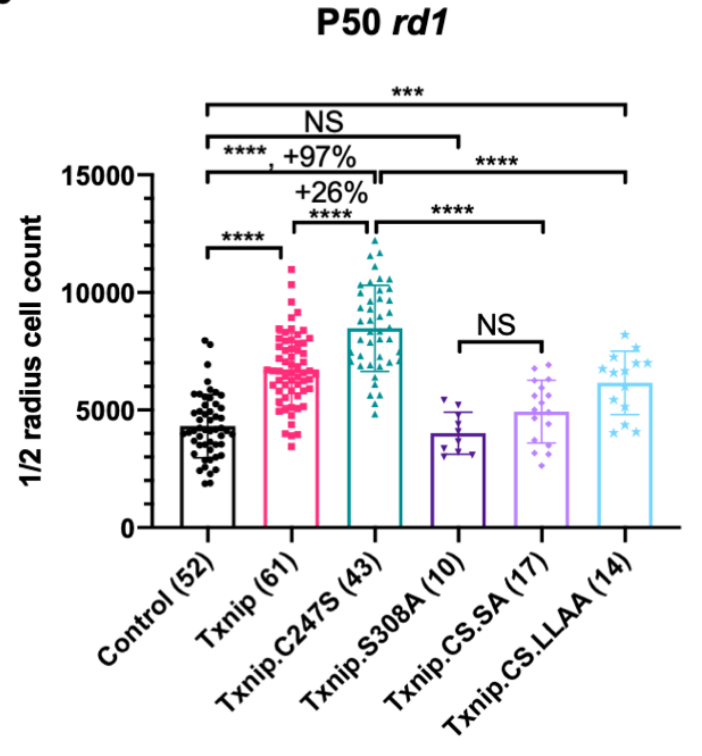


371 Fig. 3: Ldhb is necessary for Txnip-induced rescue of RP cones in vivo.

$\exists 72$ a. Representative P50 rd1 flat-mounted retinas with H2BGFP (gray) labeled cones treated with siNC (non-targeting scrambled control shRNA), siLdhb ${ }^{(\# 2)}$ (Ldhb shRNA), Txnip + siNC, or Txnip + $\operatorname{siLdhb}^{(\# 2)}$.

b. Quantification of H2BGFP-positive cones within the half radius of P50 rd1 retinas treated with control, siNC control, Txnip + siLdhb( ${ }^{(\# 2)}$ or siNC control.

c. Quantification of H2BGFP-positive cones within the half radius of P50 rd1 retinas treated with

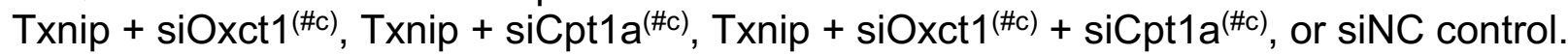
0.0001. 
a

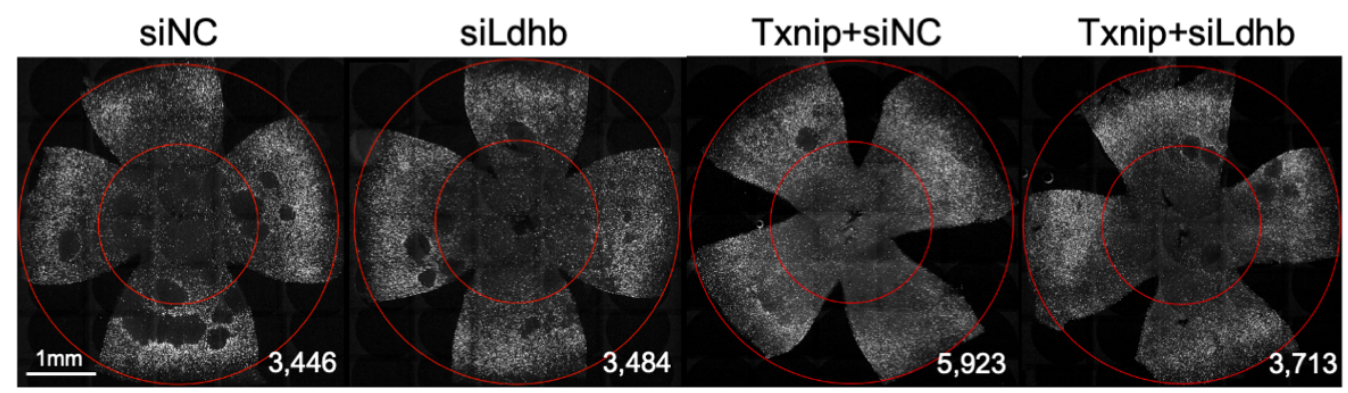

b

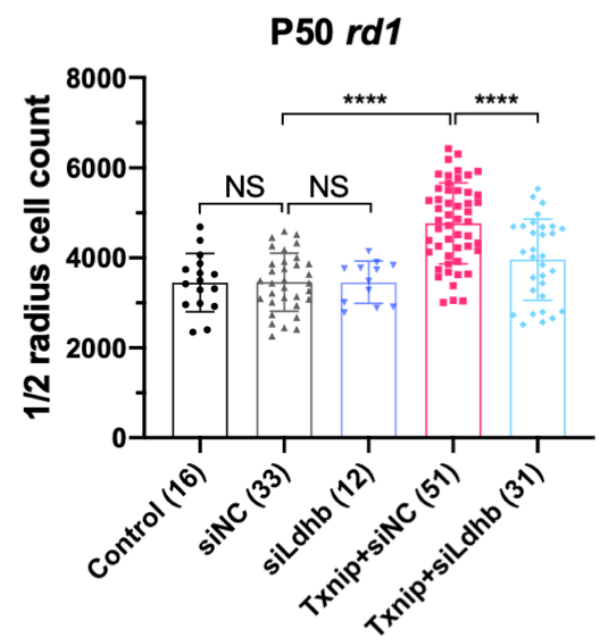

C

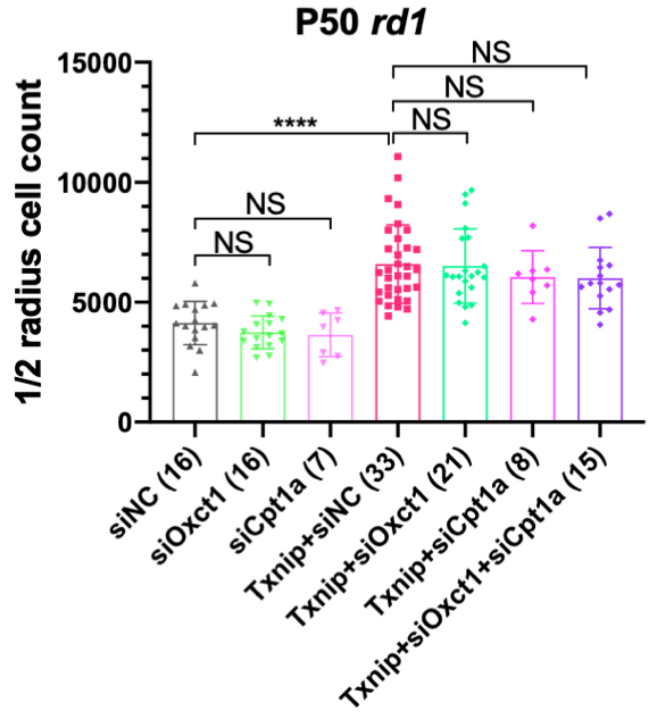


a. Representative ex vivo live images of PercevalHR labeled cones in P20 rd1 retinas cultured with high-glucose, lactate-only, or pyruvate-only medium and treated with Txnip or control. (Magenta: fluorescence by $405 \mathrm{~nm}$ excitation, indicating low-ATP:ADP. Green: fluorescence by $488 \mathrm{~nm}$ excitation, indicating high-ATP:ADP.)

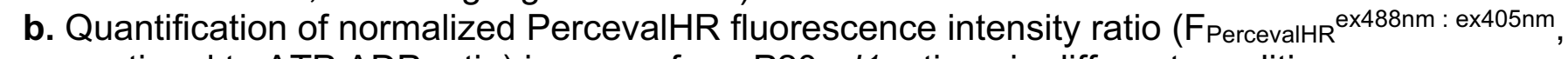
proportional to ATP:ADP ratio) in cones from P20 rd1 retinas in different conditions.

The number in the bracket "[ ]" indicates the sample size, i.e. the number of images taken from regions of interest of multiple retinas ( $\approx 3$ images per retina), in each condition (same applies to all other figures).

c. Quantification of normalized PercevalHR fluorescence intensity of Txnip + siLdhb ${ }^{(\# 2)}$ and Txnip + siNC in cones from P20 rd1 retina in lactate-only or pyruvate-only medium.

d. Representative ex vivo live images of PercevalHR labeled cones in P20 rd1 retinas cultured in lactate-only medium, following treatment with Txnip.C247S or Txnip.S308A. (Magenta:

fluorescence by $405 \mathrm{~nm}$ excitation, indicating low-ATP:ADP. Green: fluorescence by $488 \mathrm{~nm}$ excitation, indicating high-ATP:ADP.)

e. Quantification of normalized PercevalHR fluorescence intensity following treatment by Txnip, Txnip alleles, and control cones in $P 20$ rd1 retinas cultured in lactate-only medium. Abbreviations: Txnip.CS = Txnip.C247S; Txnip.SA = Txnip.S308A.

Error bar: standard deviation. NS: not significant, $p>0.05 .{ }^{* *} p<0.01 .{ }^{* * *} p<0.001 .{ }^{* * * *} p<$ or $<<$ 0.0001 . 
bioRxiv preprint doi: https://doi.org/10.1101/2021.01.27.428411; this version posted January 27, 2021. The copyright holder for this preprint (which was not certified by peer review) is the author/funder, who has granted bioRxiv a license to display the preprint in perpetuity. It is made available under aCC-BY 4.0 International license.

a

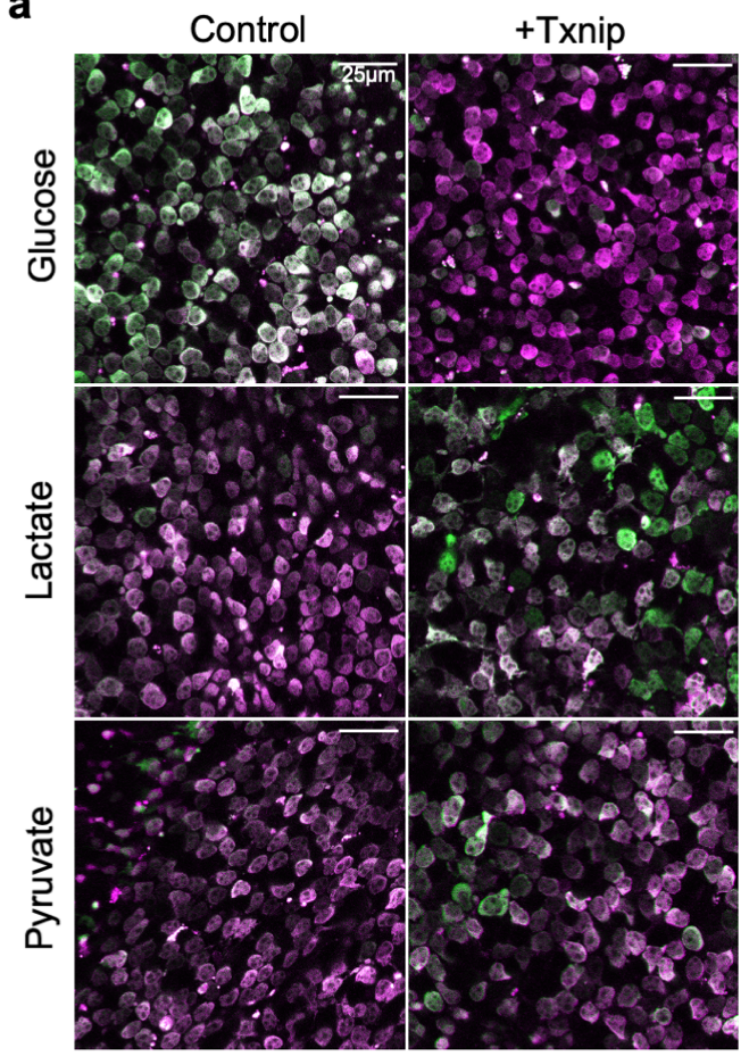

d

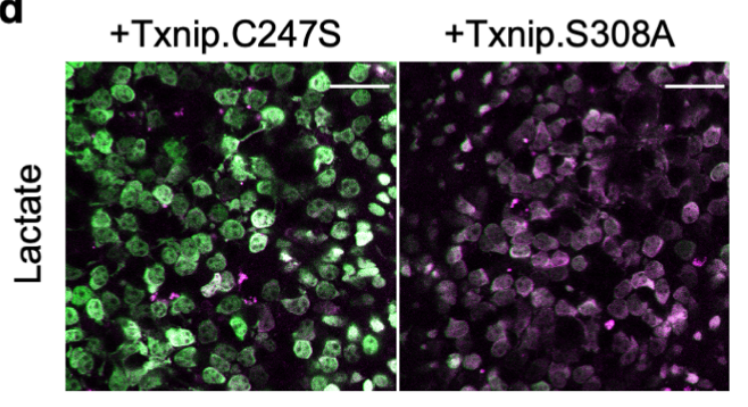

b
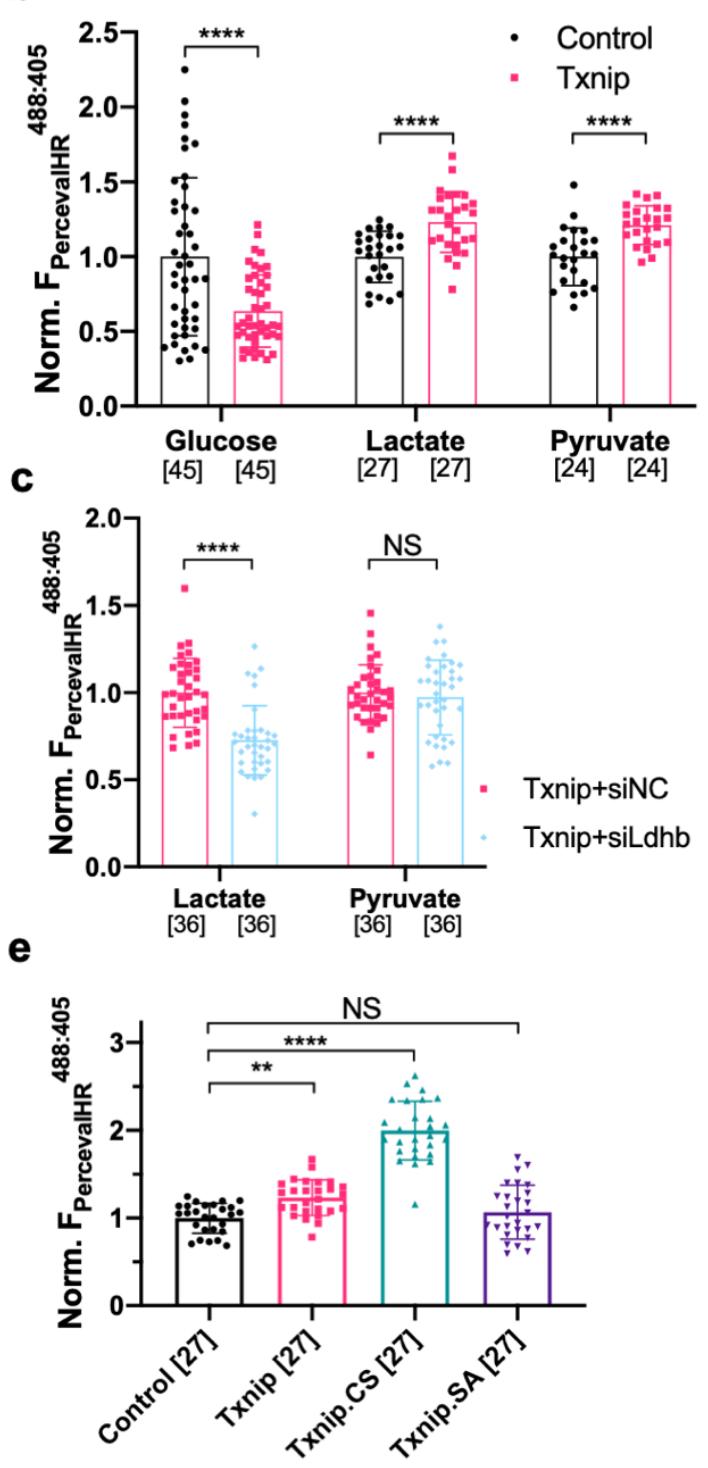


\section{Fig. 5: Txnip enhances RP cone mitochondrial size and function}

a. Representative EM images of RP cones from P20 rd1 cones treated with Txnip, Txnip.C247S, Txnip.S308A, and control.

b. Quantification of mitochondrial diameters from control, Txnip, Txnip.C247S and Txnip.S308A treated cones.

The number in the curly bracket " \{\} " indicates the sample size, i.e. the number of mitochondria from multiple cones of $\geq$ one retina for each condition ( 5 retinas for control, 4 for Txnip, 2 for Txnip.C247S, and 1 for Txnip.S308A).

c. Images of JC-1 dye staining (indicator of ETC function) in live cones of P20 rd1 central retina at different conditions. (Magenta: J-aggregate, indicating high ETC function. Green: JC-1 monomer, for self-normalization. H2BGFP channel, the tracer of AAV infected area, is not shown.)

d. Quantification of normalized cone JC-1 dye staining (fluorescence intensity of J-aggregate:JC-1 monomer) from live cones in P20 rd1 retinas in different conditions (3 - 4 images per retina).

e. Images of mitoRFP staining (reflecting mitochondrial function) in Txnip.C247S and control

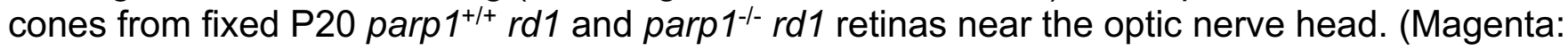
mitoRFP. Gray: H2BGFP, for mitoRPF normalization.)

f. Quantification of normalized mito-RFP:H2BGFP intensity in different conditions of P20 parp1 rd1 retinas (4 images per retina, near optical nerve head).

g. Images of P50 parp $1^{+/+}$rd1 and parp1 ${ }^{-/-}$rd1 retinas with H2BGFP (gray) labeled cones treated with Txnip.C247S or control. Rd1 cone degeneration seems to be faster after being crossed to parp1 mice (on 129S background) due to unknown reason(s). Please enlarge the images to appreciate the improved cone counts near the inner circle.

h. Quantification of H2BGFP-positive cones within the half radius of P50 parp $1^{+/+}$rd1 and parp $1^{-/-}$ rd1 retinas treated with Txnip.C247S or control.

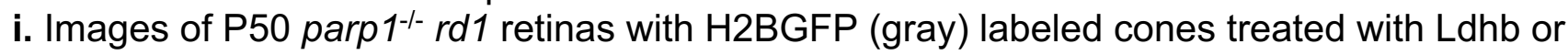
H2BGFP only.

j. Quantification of H2BGFP-positive cones within the half radius of P50 parp $1^{-/-}$rd1 retinas treated with Ldhb or H2BGFP only.

Error bar: standard deviation. NS: not significant, $p>0.05 .{ }^{*} p<0.05 .{ }^{* *} p<0.01 .{ }^{* * *} p<0.001$. ${ }^{* * * *} p<$ or $<<0.0001$. Abbreviations: Txnip.SA = Txnip; Txnip.SA = Txnip.S308A. 
a

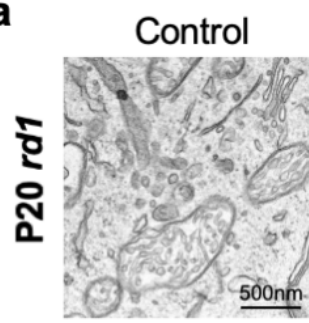

C

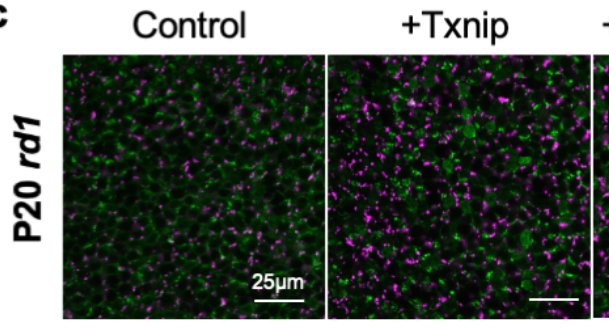

e

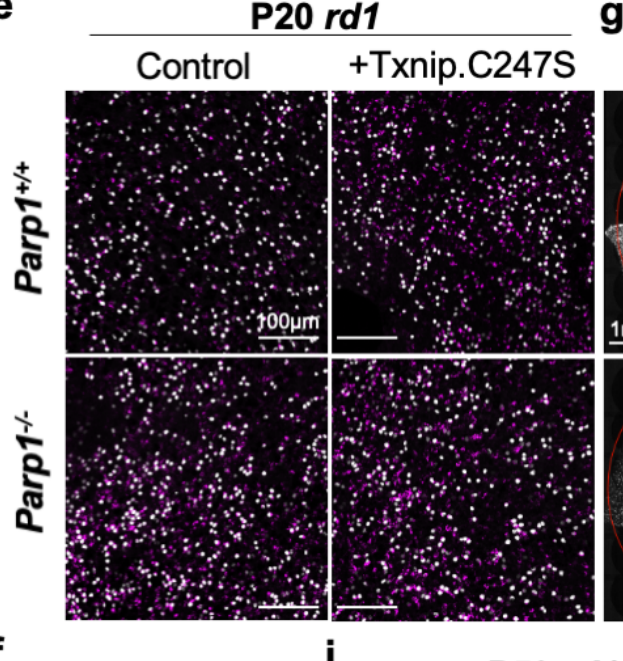

g

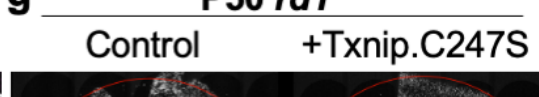

+Txnip.C247S +Txnip.S308A

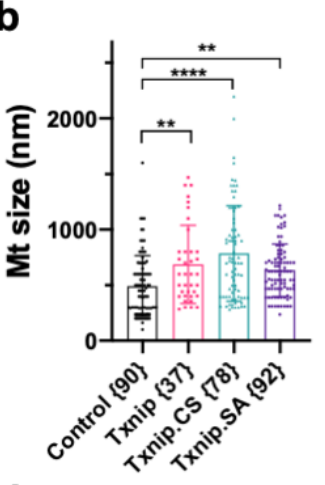

d

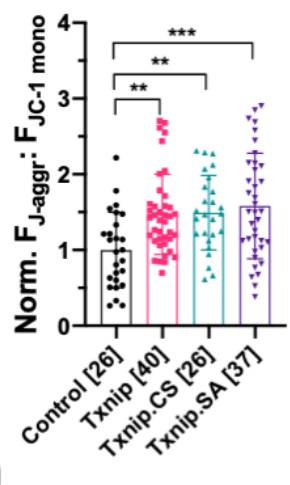

h
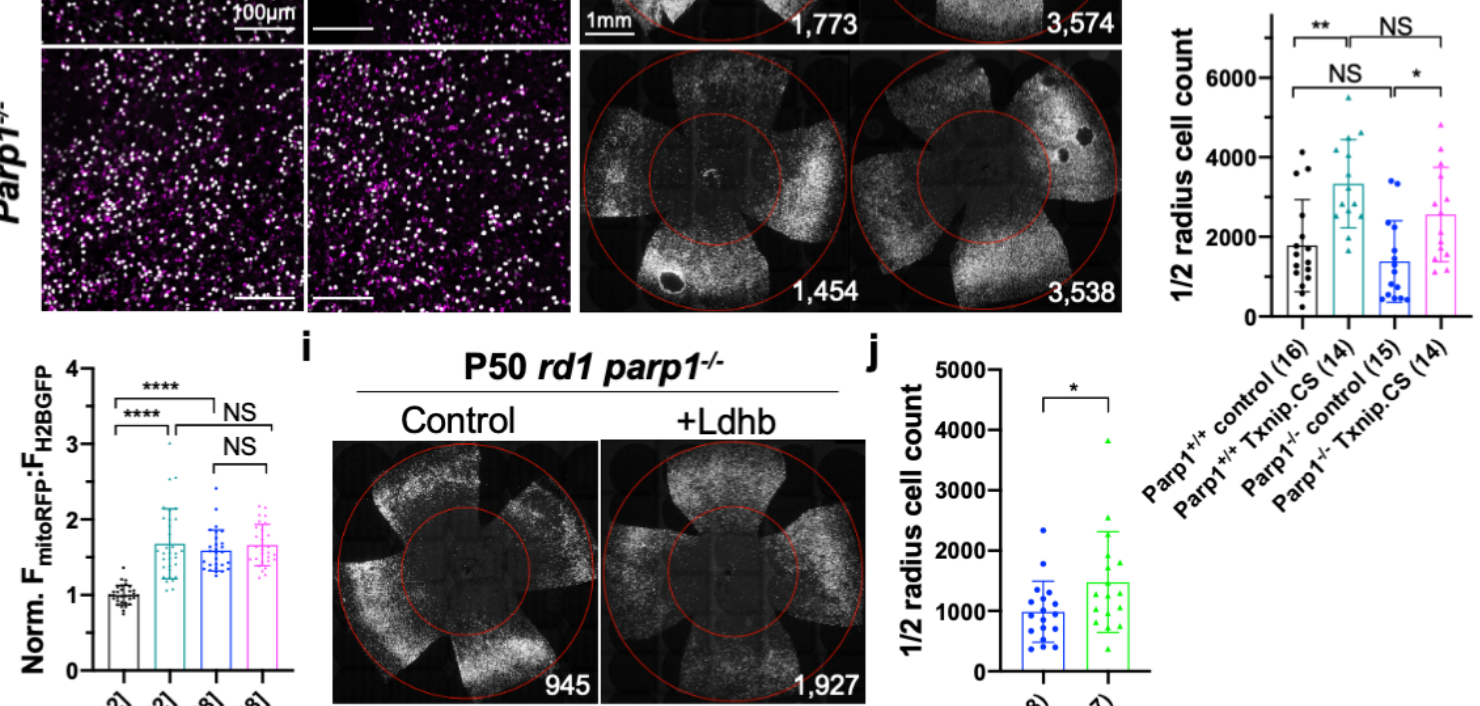

P50 rd1 parp1/-

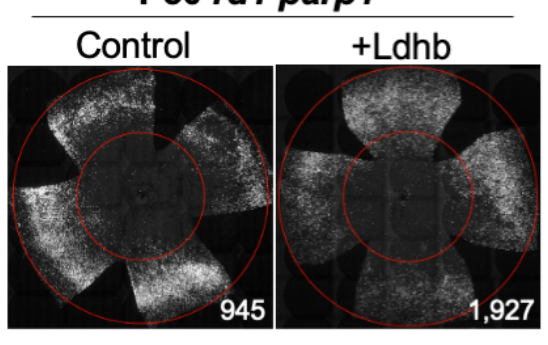

\section{j}

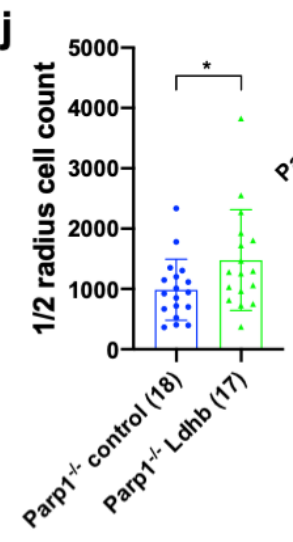


J47 Fig. 6: Txnip enhances $\mathrm{Na}^{+} / \mathrm{K}^{+}$ATPase pump function and cone opsin expression in $\mathrm{RP}$ 348 cones.

$J 49$ a. Images of live ex vivo RH421 stained cones in P20 rd1 retinas treated with Txnip.C247S or 350 control and cultured in lactate-only medium. (Magenta: RH421 fluorescence, proportional to $J 51 \quad \mathrm{Na}^{+} / \mathrm{K}^{+}$ATPase function. Gray: H2BGFP, tracer of infection).

552 b. Quantification of normalized RH421 fluorescence intensity from Txnip.C247S treated cones 553 relative to control in P20 rd1 retinas cultured in lactate-only medium (5 images per retina).

554 Abbreviation: Txnip.CS $=$ Txnip.C247S.

355 c. IHC with anti-s-opsin plus anti-m-opsin antibodies near the half radius of P50 $r d 1$ retinas treated J56 with Txnip.C247S or control. (Green: cone-opsins. Gray: H2BGFP, tracer of infection).

057 Error bar: standard deviation. ${ }^{* * * *} p<$ or $<<0.0001$.

358

359 
bioRxiv preprint doi: https://doi.org/10.1101/2021.01.27.428411; this version posted January 27, 2021. The copyright holder for this preprint (which was not certified by peer review) is the author/funder, who has granted bioRxiv a license to display the preprint in perpetuity. It is made available under aCC-BY 4.0 International license.

a

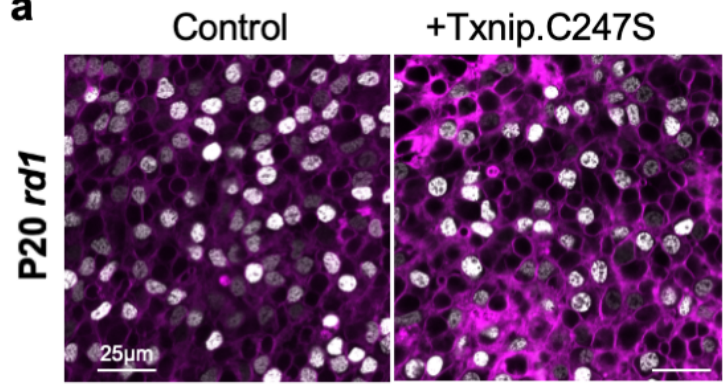

b

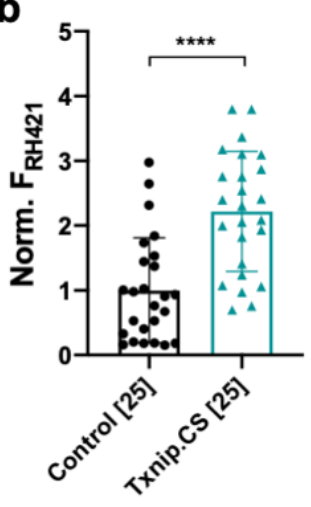

C

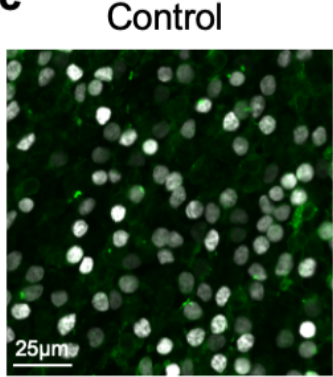

+Txnip.C247S

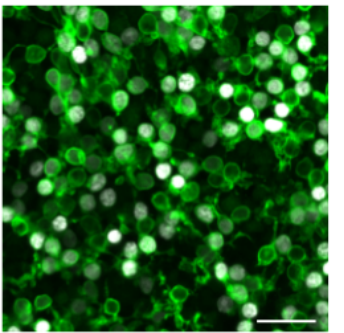


J63 Fig. 7: Dominant negative HIF1 $\alpha$ and Best1-Txnip.C247S.LL351\&352AA enhance RP cone 364 survival.

365 a. Images of P50 rd1 retinas with H2BGFP (gray) labeled cones treated with dnHIF1a, Hif1a, J66 Best1-Txnip.C247S.LL351\&352AA (Txnip.CS.LLAA, driven by an RPE-specific promoter) or $J 67$ control. Note that Best1-Txnip.C247S.LL351\&352AA amplified the FVB-specific retinal craters, J68 while dnHIF1a decreased them.

369 b. Quantification of H2BGFP-positive cones within the half radius of P50 rd1 retinas treated with 370 dnHIF1a, Hif1a, Best1-Txnip.C247S.LL351\&352AA or control. Abbreviation: B-Tx.CS.LLAA = J71 Best1-Txnip.C247S.LL351\&352AA.

372 Error bar: standard deviation. ${ }^{*} p<0.05 .{ }^{* *} p<0.01 .{ }^{* * *} p<0.001$. 
bioRxiv preprint doi: https://doi.org/10.1101/2021.01.27.428411; this version posted January 27, 2021. The copyright holder for this preprint (which was not certified by peer review) is the author/funder, who has granted bioRxiv a license to display the preprint in perpetuity. It is made available under aCC-BY 4.0 International license.

a

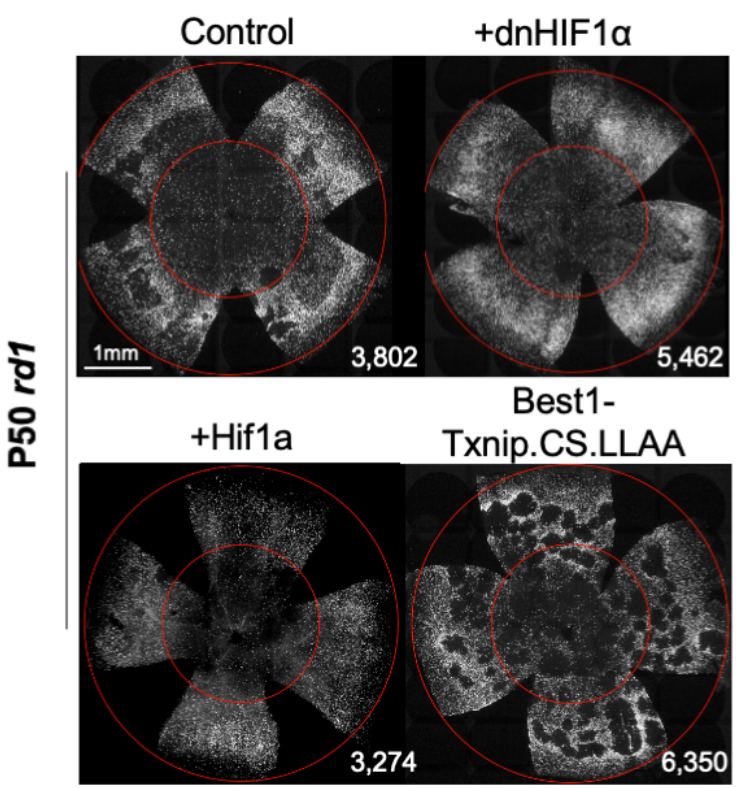

b

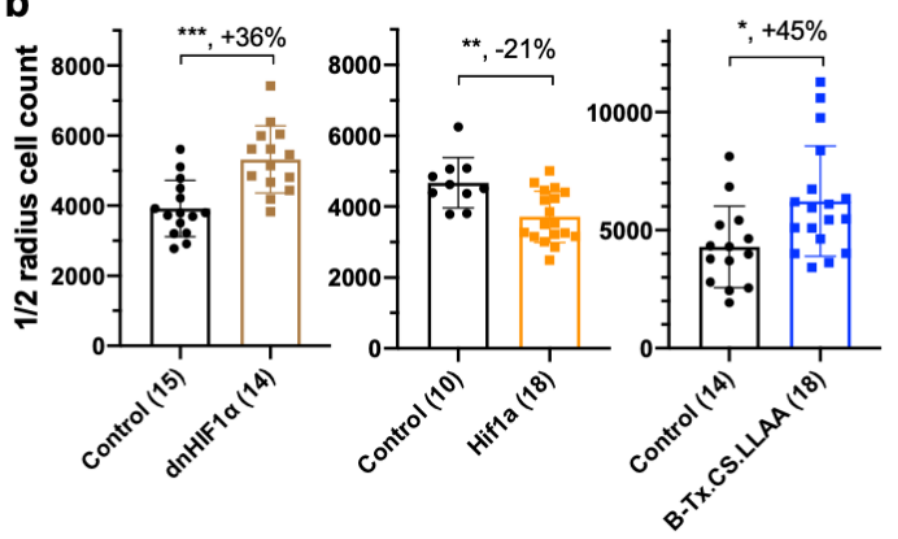


J76 Fig. 8: Combination of Txnip.C247S with Best1-Nrf2 or Tgfb1 provides an additive effect. $J 77$ a. Images of P50 rd1 retinas with H2BGFP (gray) labeled cones treated with Txnip.C247S or 378 Txnip.C247S + Best1-Nrf2.

379 b. Quantification of H2BGFP-positive cones within the half radius of P50 rd1 retinas treated with 380 Txnip.C247S or Txnip.C247S + Best1-Nrf2. Abbreviation: B-Nrf2 = Best1-Nrf2.

381 c. IHC with anti-s-opsin plus anti-m-opsin antibodies near the half radius of $\mathrm{P} 130 \mathrm{rd} 10$ retinas treated with Txnip.C247S (left panel) or Txnip.C247S + Best1-Nrf2 (right panel). (Green: coneopsins. Gray: H2BGFP, tracer of infection).

d. Images of P50 rd1 retinas with H2BGFP (gray) labeled cones treated with Txnip.C247S or Txnip.C247S + Tgfb1.

e. Quantification of H2BGFP-labeled cones within the half radius of P50 rd1 retinas treated with control, Tgfb1, Txnip.C247S, or Txnip.C247S + Tgfb1. 
bioRxiv preprint doi: https://doi.org/10.1101/2021.01.27.428411; this version posted January 27, 2021. The copyright holder for this preprint (which was not certified by peer review) is the author/funder, who has granted bioRxiv a license to display the preprint in perpetuity. It is made available under aCC-BY 4.0 International license.

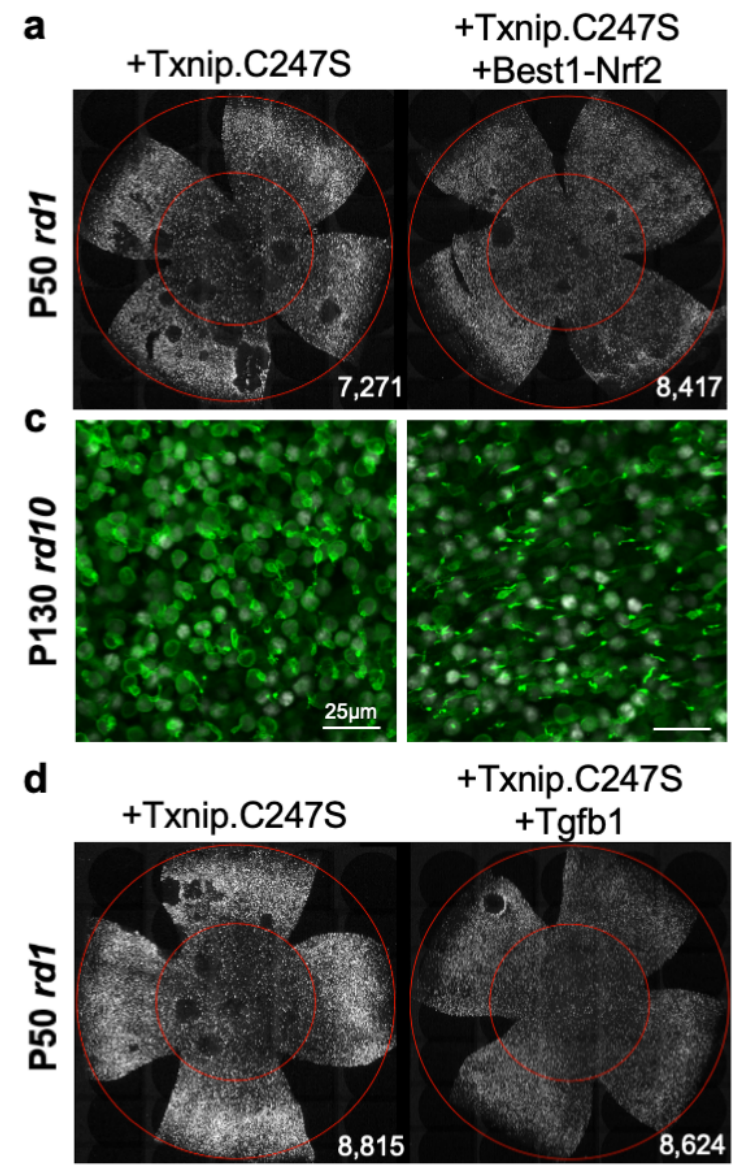

b

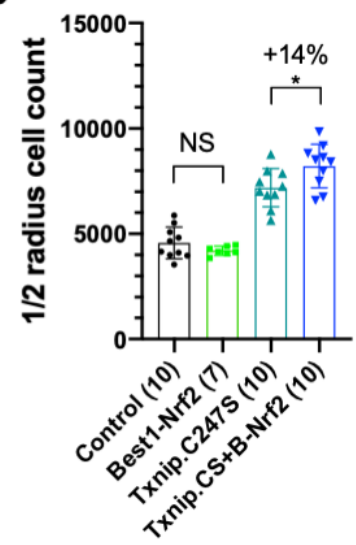

e

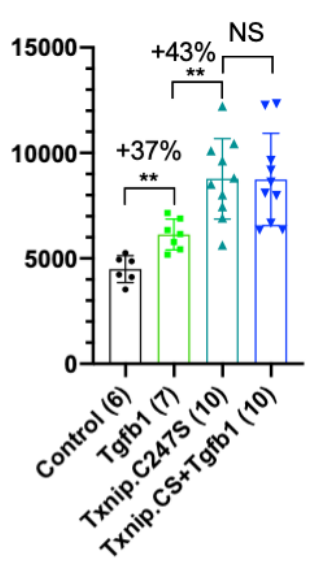

Fig. 8 
bioRxiv preprint doi: https://doi.org/10.1101/2021.01.27.428411; this version posted January 27, 2021. The copyright holder for this preprint

(which was not certified by peer review) is the author/funder, who has granted bioRxiv a license to display the preprint in perpetuity. It is made available under aCC-BY 4.0 International license.

\section{J91 Supplementary Table 1: AAV8 vectors used in this study.}

\begin{tabular}{|c|c|c|c|c|c|c|c|c|c|c|c|c|}
\hline Inserts & $\begin{array}{c}\text { Insert } \\
\text { species }\end{array}$ & Promoter & Intron & WPRE & poly (A) & $\begin{array}{l}\text { ITR-to- } \\
\text { ITR size }\end{array}$ & $\begin{array}{c}\text { Digestion } \\
(\text { Xmal, ITR) }\end{array}$ & $\begin{array}{c}\text { Partial seq } \\
\text { (ligation site) }\end{array}$ & $\begin{array}{c}\text { Complete } \\
\text { plasmid } \\
\text { seq }\end{array}$ & $\begin{array}{c}\text { AAV } \\
\text { genome } \\
\text { seq }\end{array}$ & Efficacy & $\begin{array}{l}\text { Raw } \\
\text { data }\end{array}$ \\
\hline H2BGFP & $\mathrm{N} / \mathrm{A}$ & RedO (default) & $\mathrm{N} / \mathrm{A}$ & WPRE & Bovine GH & $4.4 \mathrm{~kb}$ & Correct & Correct & - & - & $\mathrm{N} / \mathrm{A}$ & - \\
\hline H2BGFP & $\mathrm{N} / \mathrm{A}$ & SynP136 & N/A & WPRE3 & SV40-Late & $3.9 \mathrm{~kb}$ & Correct & Correct & - & - & $\mathrm{N} / \mathrm{A}$ & - \\
\hline mitoRFP & N/A & SynP136 & N/A & WPRE3 & SV40-Late & $3.5 \mathrm{~kb}$ & Correct & Correct & Correct & - & $\mathrm{N} / \mathrm{A}$ & - \\
\hline Txnip & Mouse & RedO (default) & N/A & WPRE & Bovine GH & $4.5 \mathrm{~kb}$ & Correct & Correct & Correct & - & Pos & Fig. 1 \\
\hline Txnip & Mouse & SynPVI & N/A & WPRE3 & SV40-Late & $2.4 \mathrm{~kb}$ & Correct & Correct & - & - & Pos & Fig. S1 \\
\hline Txnip & Mouse & Best1 & SV40 & WPRE & Bovine GH & $3.1 \mathrm{~kb}$ & Correct & Correct & - & - & $\mathrm{Neg}$ & Fig. S6 \\
\hline Txnip.C247S & Mouse & RedO & N/A & WPRE & Bovine GH & $4.5 \mathrm{~kb}$ & Correct & Correct & Correct & Correct & Pos & Fig. 2 \\
\hline Txnip.S308A & Mouse & RedO & N/A & WPRE & Bovine GH & $4.5 \mathrm{~kb}$ & Correct & Correct & Correct & Correct & $\mathrm{Neg}$ & Fig. 2 \\
\hline Txnip.C247S.S308A & Mouse & RedO & N/A & WPRE & Bovine GH & $4.5 \mathrm{~kb}$ & Correct & Correct & Correct & - & $\mathrm{Neg}$ & Fig. 2 \\
\hline $\begin{array}{c}\text { Txnip.C247S.LL351\& } \\
\text { 352AA } \\
\end{array}$ & Mouse & RedO & N/A & WPRE & Bovine GH & $4.5 \mathrm{~kb}$ & Correct & Correct & Correct & - & Pos & Fig. 2 \\
\hline $\begin{array}{c}\text { Txnip.C247S.LL351\& } \\
\text { 352AA }\end{array}$ & Mouse & Best1 & SV40 & WPRE & Bovine GH & $3.1 \mathrm{~kb}$ & Correct & Correct & Correct & - & Pos & Fig. 7 \\
\hline GFP-Txnip & Mouse & $\mathrm{RO1.7}$ & N/A & WPRE3 & SV40-Late & $4.4 \mathrm{~kb}$ & Correct & Correct & Correct & - & N/A & - \\
\hline Nrf2 & Human & CMV & $\begin{array}{c}\text { human } \beta- \\
\text { globin }\end{array}$ & $\mathrm{N} / \mathrm{A}$ & SV40 & $3.7 \mathrm{~kb}$ & - & - & - & - & Pos & Fig. S1 \\
\hline Nrf2 & Human & SynPVI & N/A & WPRE3 & SV40-Late & $3.1 \mathrm{~kb}$ & Correct & Correct & Correct & - & $\mathrm{Neg}$ & Fig. S6 \\
\hline Nrf2 & Human & Best1 & SV40 & WPRE & Bovine GH & $3.8 \mathrm{~kb}$ & Correct & Correct & - & - & $\mathrm{Neg}$ & Fig. 8 \\
\hline $\mathrm{Cx} 3 \mathrm{cl} 1$ & Mouse & Best1 & SV40 & WPRE & Bovine GH & $3.0 \mathrm{~kb}$ & - & Correct & Correct & - & Pos & Fig. S6 \\
\hline $\mathrm{Cx} 3 \mathrm{cl} 11$ & Mouse & RedO & N/A & WPRE & Bovine GH & $4.3 \mathrm{~kb}$ & - & Correct & - & - & $\mathrm{Neg}$ & Fig. S6 \\
\hline Tgfb1 & Mouse & RedO & N/A & WPRE & Bovine GH & $4.4 \mathrm{~kb}$ & - & Correct & - & - & Pos & Fig. 8 \\
\hline dnHIF1a & Mouse & R01.7 & N/A & WPRE3 & SV40-Late & $3.6 \mathrm{~kb}$ & Correct & Correct & Correct & - & Pos & Fig. 7 \\
\hline dnHIF1a & Mouse & Best1 & SV40 & WPRE & Bovine GH & $3.1 \mathrm{~kb}$ & Correct & Correct & Correct & - & $\mathrm{Neg}$ & Fig. S6 \\
\hline Hif1a & Mouse & SynPVI & N/A & WPRE3 & SV40-Late & $3.8 \mathrm{~kb}$ & Correct & Correct & Correct & - & $\mathrm{Neg}$ & Fig. 7 \\
\hline Hk1 & Human & SynPVI & N/A & WPRE3 & SV40-Late & $4.0 \mathrm{~kb}$ & Correct & Correct & - & - & $\mathrm{Neg}$ & Fig. S1 \\
\hline Hk2 & Mouse & SynPVI & N/A & WPRE3 & SV40-Late & $4.0 \mathrm{~kb}$ & Correct & Correct & - & - & $\mathrm{Neg}$ & Fig. S1 \\
\hline Pfkm & Human & SynPVI & N/A & WPRE3 & SV40-Late & $3.6 \mathrm{~kb}$ & Correct & Correct & - & - & $\mathrm{Neg}$ & Fig. S1 \\
\hline Pkm1 & Human & SynPVI & N/A & WPRE3 & SV40-Late & $2.8 \mathrm{~kb}$ & Correct & Correct & - & - & $\mathrm{Neg}$ & Fig. S1 \\
\hline Pkm2 & Human & SynPVI & N/A & WPRE3 & SV40-Late & $2.8 \mathrm{~kb}$ & Correct & Correct & - & - & $\mathrm{Neg}$ & Fig. S1 \\
\hline Ldha & Mouse & $\mathrm{RO1.7}$ & N/A & WPRE3 & SV40-Late & $3.6 \mathrm{~kb}$ & Correct & Correct & Correct & - & $\mathrm{Neg}$ & Fig. S1 \\
\hline Ldhb & Mouse & RedO & N/A & WPRE & Bovine GH & $4.3 \mathrm{~kb}$ & Correct & Correct & Correct & - & $\mathrm{Neg}$ & Fig. S6 \\
\hline Ldhb-3xFLAG & Mouse & R01.7 & N/A & WPRE3 & SV40-Late & $3.6 \mathrm{~kb}$ & Correct & Correct & Correct & - & N/A & - \\
\hline SIc2a1 & Mouse & R01.7 & N/A & WPRE3 & SV40-Late & $4.0 \mathrm{~kb}$ & Correct & Correct & Correct & - & $\mathrm{Neg}$ & Fig. S1 \\
\hline Bsg1 & Mouse & RedO & N/A & WPRE & Bovine GH & $4.4 \mathrm{~kb}$ & Correct & Correct & - & - & $\mathrm{Neg}$ & Fig. S1 \\
\hline RdCVF & Mouse & RedO & N/A & WPRE & Bovine GH & $3.6 \mathrm{~kb}$ & Correct & Correct & - & - & $\mathrm{Neg}$ & Fig. S1 \\
\hline RdCVF & Mouse & Best1 & SV40 & WPRE & Bovine GH & $2.3 \mathrm{~kb}$ & Correct & Correct & - & - & $\mathrm{Neg}$ & Fig. S6 \\
\hline Cpt1a & Mouse & RedO & N/A & WPRE & Bovine GH & $5.6 \mathrm{~kb}$ & Correct & Correct & Correct & - & $\mathrm{Neg}$ & Fig. S1 \\
\hline Oxct1 & Mouse & RedO & N/A & WPRE & Bovine GH & $4.8 \mathrm{~kb}$ & Correct & Correct & Correct & - & $\mathrm{Neg}$ & Fig. S1 \\
\hline Mpc1 & Mouse & R01.7 & N/A & WPRE3 & SV40-Late & $3.0 \mathrm{~kb}$ & Correct & Correct & - & - & $\mathrm{Neg}$ & Fig. S6 \\
\hline Mpc2 & Mouse & R01.7 & N/A & WPRE3 & SV40-Late & $2.9 \mathrm{~kb}$ & Correct & Correct & - & - & $\mathrm{Neg}$ & Fig. S6 \\
\hline Vegf164 & Mouse & R01.7 & N/A & WPRE3 & SV40-Late & $3.1 \mathrm{~kb}$ & Correct & Correct & Correct & - & $\mathrm{Neg}$ & Fig. S6 \\
\hline PercevalHR & $\mathrm{N} / \mathrm{A}$ & $\mathrm{RO1.7}$ & N/A & WPRE3 & SV40-Late & $4.2 \mathrm{~kb}$ & Correct & Correct & Correct & - & $\mathrm{N} / \mathrm{A}$ & - \\
\hline iGlucoSnFR & $\mathrm{N} / \mathrm{A}$ & SynPVI & N/A & WPRE3 & SV40-Late & $4.0 \mathrm{~kb}$ & Correct & Correct & Correct & - & N/A & - \\
\hline pHRed & $\mathrm{N} / \mathrm{A}$ & SynP136 & N/A & WPRE3 & SV40-Late & $3.5 \mathrm{~kb}$ & Correct & Correct & Correct & - & N/A & - \\
\hline siNC & $\mathrm{N} / \mathrm{A}$ & RedO & N/A & WPRE & Bovine GH & $3.3 \mathrm{~kb}$ & Correct & Correct & Correct & - & $\mathrm{Neg}$ & Fig. 3 \\
\hline siLdhb $^{(\# 2)}$ (default) & Mouse & RedO & N/A & WPRE & Bovine GH & $3.3 \mathrm{~kb}$ & Correct & Correct & Correct & - & $\mathrm{Neg}$ & Fig. 3 \\
\hline siLdhb $^{(\# 1)}$ & Mouse & RedO & N/A & WPRE & Bovine GH & $3.3 \mathrm{~kb}$ & Correct & Correct & Correct & - & - & - \\
\hline sildahb(\#3) & Mouse & RedO & N/A & WPRE & Bovine GH & $3.3 \mathrm{~kb}$ & Correct & Correct & Correct & - & - & - \\
\hline siOxct1 & Mouse & RedO & N/A & WPRE & Bovine GH & $3.3 \mathrm{~kb}$ & Correct & Correct & Correct & - & $\mathrm{Neg}$ & Fig. 3 \\
\hline siCpt1a & Mouse & RedO & N/A & WPRE & Bovine GH & $3.3 \mathrm{~kb}$ & Correct & Correct & Correct & - & $\mathrm{Neg}$ & Fig. 3 \\
\hline siSlc2a1 & Mouse & RedO & N/A & WPRE & Bovine GH & $3.3 \mathrm{~kb}$ & Correct & Correct & Correct & - & $\mathrm{Neg}$ & Fig. S2 \\
\hline
\end{tabular}


bioRxiv preprint doi: https://doi.org/10.1101/2021.01.27.428411; this version posted January $27,2021$. The copyright holder for this preprint (which was not certified by peer review) is the author/funder, who has granted bioRxiv a license to display the preprint in perpetuity. It is made available under aCC-BY 4.0 International license.

J93 Supplementary Table 2: Differentially expressed genes in common between $r d 1$ and $r \mathbf{r o}^{-/}$ J94 cones infected by AAV-Txnip.

\begin{tabular}{|c|c|c|c|c|c|c|c|c|}
\hline & \multicolumn{4}{|c|}{ P90 rho- } & \multicolumn{4}{|c|}{ P21 rd1 } \\
\hline $\begin{array}{c}\text { MGI } \\
\text { symbol }\end{array}$ & $\begin{array}{l}\text { Base } \\
\text { Mean } \\
\end{array}$ & $\begin{array}{c}\text { log2Fold } \\
\text { Change }\end{array}$ & $\begin{array}{c}\log 2 F o l d \\
\text { SE }\end{array}$ & $\begin{array}{c}\text { Adjusted } \\
\text { p-value }\end{array}$ & $\begin{array}{l}\text { Base } \\
\text { Mean }\end{array}$ & $\begin{array}{c}\text { log2Fold } \\
\text { Change }\end{array}$ & $\begin{array}{c}\log 2 \mathrm{Fold} \\
\text { SE }\end{array}$ & $\begin{array}{c}\text { Adjusted } \\
\text { p-value }\end{array}$ \\
\hline Txnip & 1324.3 & 10.211 & 0.516 & $4.78 \mathrm{E}-83$ & 582.4 & 9.325 & 0.775 & 2.17E-29 \\
\hline mt-Cytb & 13643.7 & 1.248 & 0.257 & 0.00079349 & 5651.4 & 0.384 & 0.082 & 0.00014272 \\
\hline mt-Nd4 & 3118.0 & 1.195 & 0.212 & 3.52E-05 & 1504.6 & 0.392 & 0.076 & 1.76E-05 \\
\hline Vax2os & 67.2 & 1.028 & 0.323 & 0.04091883 & 33.6 & 0.932 & 0.321 & 0.04383248 \\
\hline mt-Co1 & 9876.4 & 0.808 & 0.189 & 0.00393386 & 5235.1 & 0.388 & 0.075 & 1.7625E-05 \\
\hline Rom1 & 5040.6 & 0.748 & 0.190 & 0.00789643 & 4432.7 & 0.164 & 0.056 & 0.04435279 \\
\hline Cd63 & 488.9 & 0.717 & 0.233 & 0.04957176 & 249.8 & 0.370 & 0.110 & 0.01303008 \\
\hline Ftl1 & 799.9 & 0.657 & 0.212 & 0.04701416 & 934.6 & 0.252 & 0.081 & 0.02671713 \\
\hline Utp14b & 51.1 & -2.486 & 0.586 & 0.00406139 & 60.1 & -1.679 & 0.246 & 2.9705E-09 \\
\hline Slc9a7 & 52.9 & -1.993 & 0.494 & 0.00646284 & 45.3 & -1.047 & 0.278 & 0.00408081 \\
\hline Megf9 & 369.0 & -1.752 & 0.529 & 0.03204053 & 417.7 & -0.666 & 0.173 & 0.00313404 \\
\hline Mgat2 & 33.4 & -1.572 & 0.434 & 0.01699743 & 36.7 & -1.343 & 0.343 & 0.00251377 \\
\hline Rnf168 & 40.0 & -1.508 & 0.476 & 0.04171633 & 45.7 & -0.970 & 0.275 & 0.00857761 \\
\hline Mid1 & 60.9 & -1.478 & 0.395 & 0.0126336 & 213.7 & -0.995 & 0.198 & 3.5144E-05 \\
\hline Ptprn2 & 333.4 & -1.461 & 0.358 & 0.00598384 & 45.8 & -0.994 & 0.309 & 0.01998408 \\
\hline Ankle2 & 115.9 & -1.429 & 0.350 & 0.00598384 & 99.1 & -0.605 & 0.207 & 0.04246472 \\
\hline Ccny & 71.1 & -1.274 & 0.379 & 0.02959143 & 74.7 & -1.034 & 0.258 & 0.00181454 \\
\hline Galnt13 & 361.3 & -1.244 & 0.341 & 0.0159734 & 504.9 & -0.371 & 0.113 & 0.01655375 \\
\hline Ablim1 & 135.7 & -1.172 & 0.296 & 0.00760665 & 158.7 & -0.798 & 0.151 & 1.1301E-05 \\
\hline AcsI3 & 460.5 & -1.075 & 0.309 & 0.0236877 & 703.9 & -1.467 & 0.153 & 3.0866E-18 \\
\hline Ube3a & 161.2 & -1.027 & 0.303 & 0.02803579 & 209.5 & -0.688 & 0.187 & 0.00513769 \\
\hline Socs 5 & 358.6 & -0.820 & 0.256 & 0.03984866 & 337.8 & -0.811 & 0.126 & 2.8137E-08 \\
\hline Heg1 & 1328.6 & -0.795 & 0.209 & 0.01057592 & 1062.7 & -0.378 & 0.067 & 1.6244E-06 \\
\hline Cand1 & 323.4 & -0.744 & 0.231 & 0.03864972 & 283.1 & -0.597 & 0.187 & 0.02106014 \\
\hline Gprasp1 & 509.6 & -0.534 & 0.163 & $|0.03426564|$ & 356.1 & -0.416 & 0.145 & 0.04690377 \\
\hline
\end{tabular}


397 Extended Data Fig. 1: Figures related to Fig. 1.

a. Schematics photoreceptor degeneration in RP mice. \# rd10 mid stage varies due to lightdependent rod degeneration (Chang et al., 2007).

b. AAV8-RO1.7-GFP.Txnip expression in P21 wt (BALB/c) and P16 rd1 retina. (Green: GFP. Magenta: PNA for cone extracellular matrix. Gray: DAPI.) Abbreviations. OS: outer segment, IS: inner segment, ONL: outer nuclear layer, OPL: outer plexiform layer, INL: inner nuclear layer, RPE: retinal pigmented epithelium.

c. Pixels recognized as cones by a MATLAB automated-counting program zoomed in from the small boxes in the top four panels (Fig. 1a). (Gray: H2BGFP labeled cones. Magenta: center of one labeled cell recognized by MATLAP program.)

d. P36 wildtype (C57BL/6J) retinal cross-section with PNA staining injected with control or 2E9 $\mathrm{vg} /$ eye RedO-Txnip, indicating RedO-Txnip is not toxic to the wildtype cones. 3E8 vg/eye RedO-H2BGFP was co-injected to track infection. (Magenta: PNA. Green: H2BGFP. Gray: DAPI.)

e. Quantification of H2BGFP-positive cones within the half radius of P20 rd1 control retinas, and P50 rd1 retinas treated with 20 different vectors and combinations or control. (Please note: we did not use dark-reared $r d 10$ for testing the RdCVF vector, and our AAV capsid and promoter were different from the original study (Byrne et al., 2015). 
a

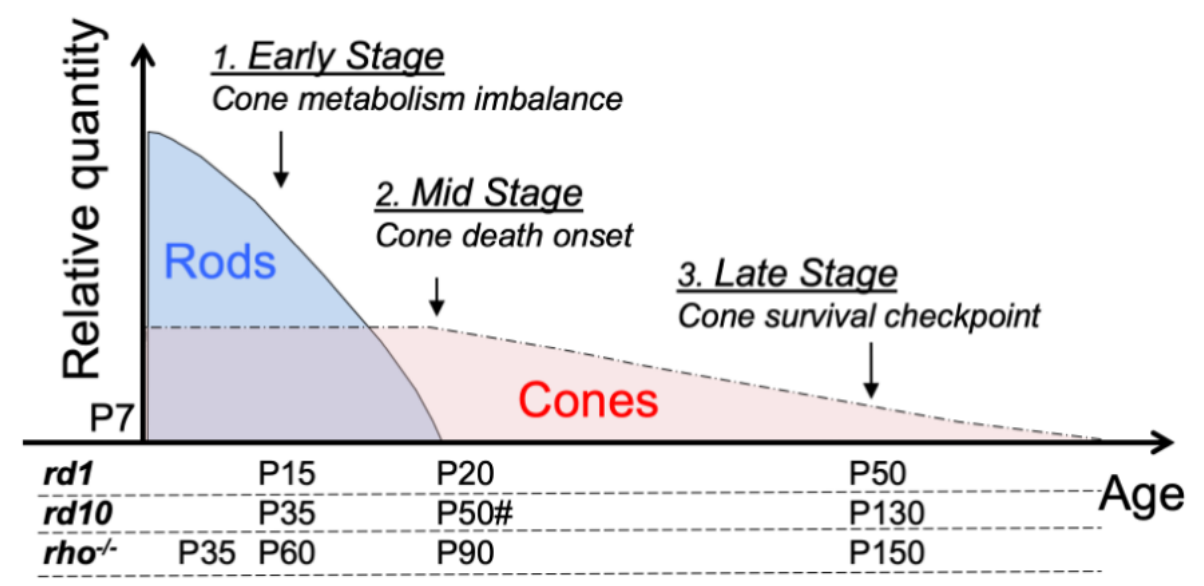

C
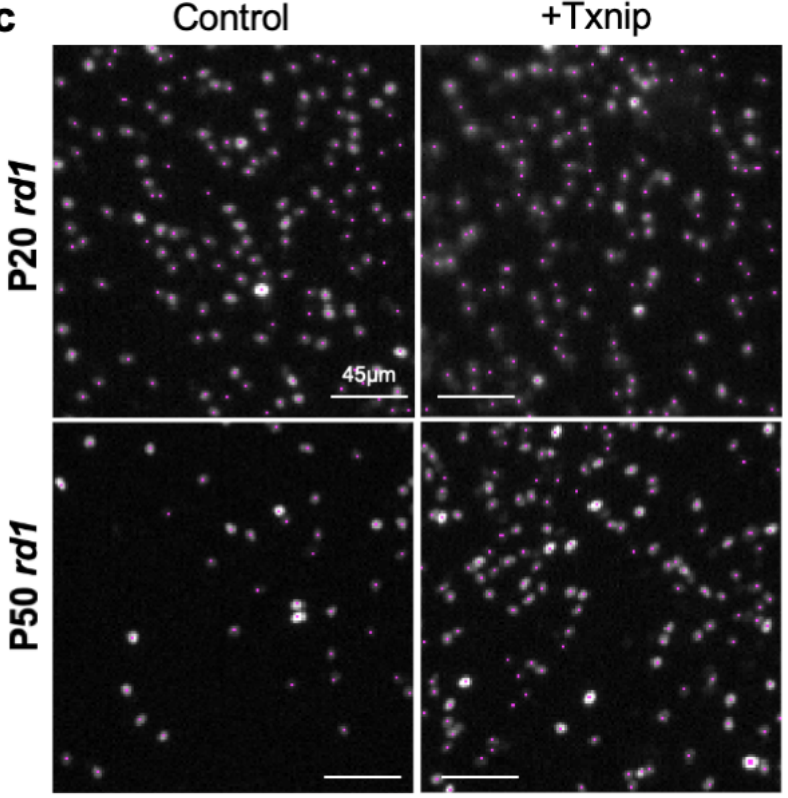

b

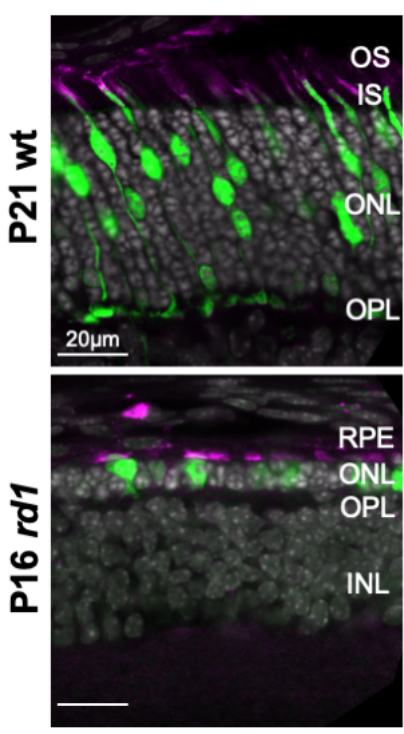

d

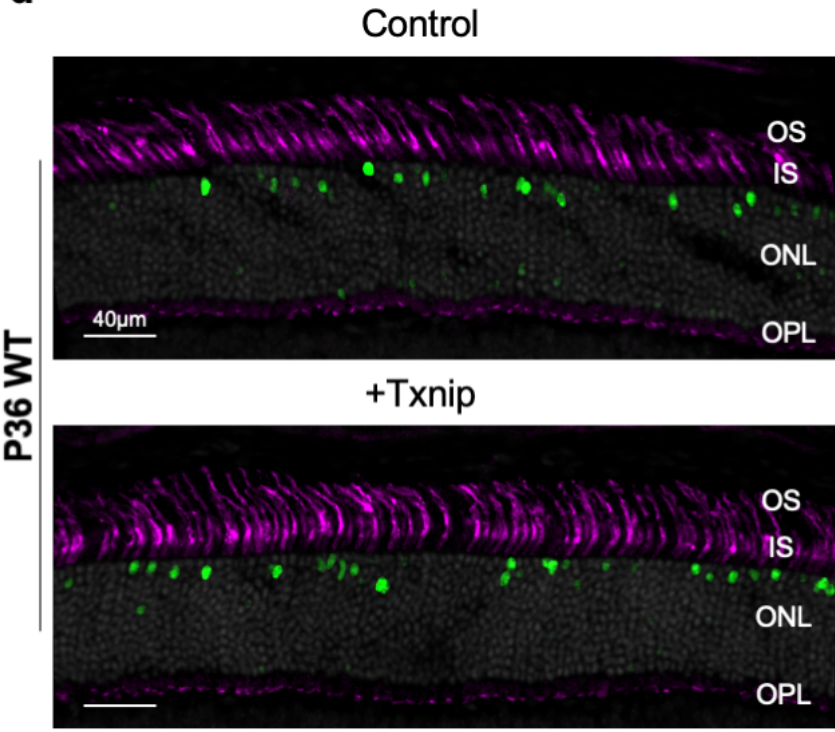

e

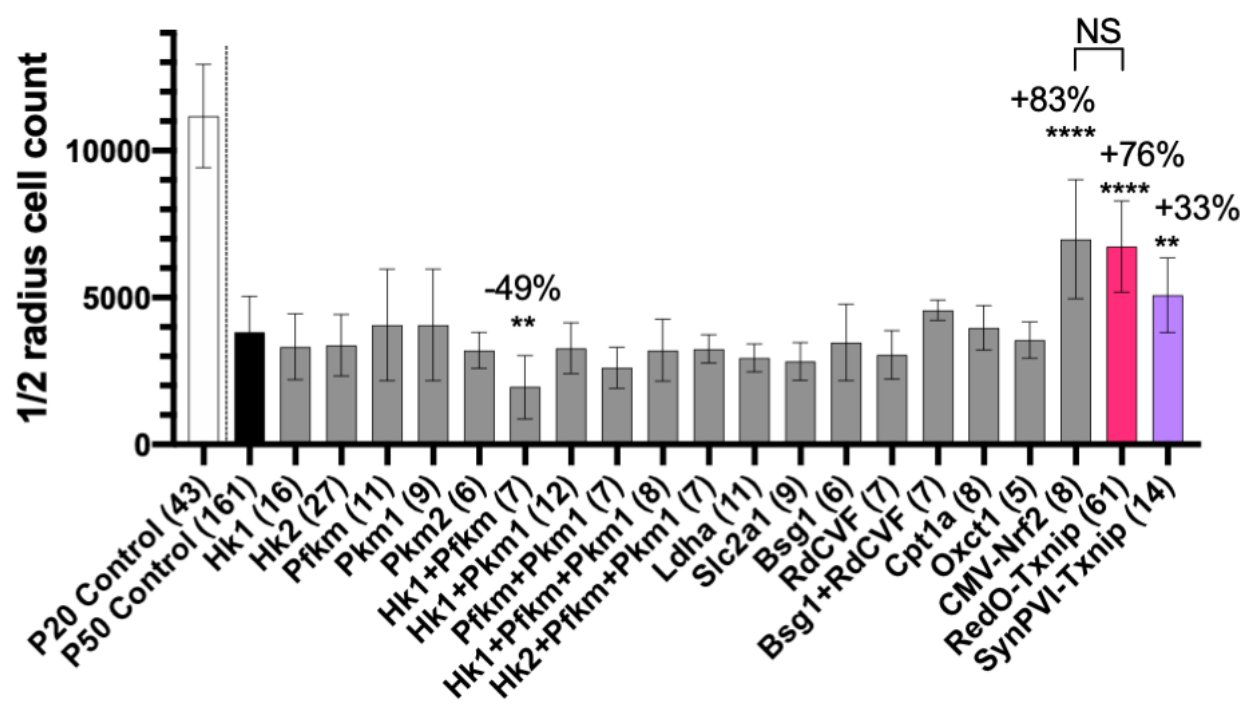




\section{Extended Data Fig. 2: Figures related to Fig. 2.}

a. Representative P130 rd10 and P150 rho-- flat-mounted retinas with H2BGFP (gray) labeled cones treated with Txnip.C247S or control.

b. Quantification of H2BGFP-positive cones within the half radius of P130 rd10 and P150 rho-/retinas treated with Txnip.C247S or control.

c. Quantification of H2BGFP-positive cones within the half radius of P20 rd1 retinas treated with Txnip, Txnip.S308A or control.

d. Quantification of H2BGFP-positive cones within the half radius of P50 rd1 retinas treated with siNC (non-targeting scrambled control shRNA) or Slc2a1/Glut1 shRNA. 
a

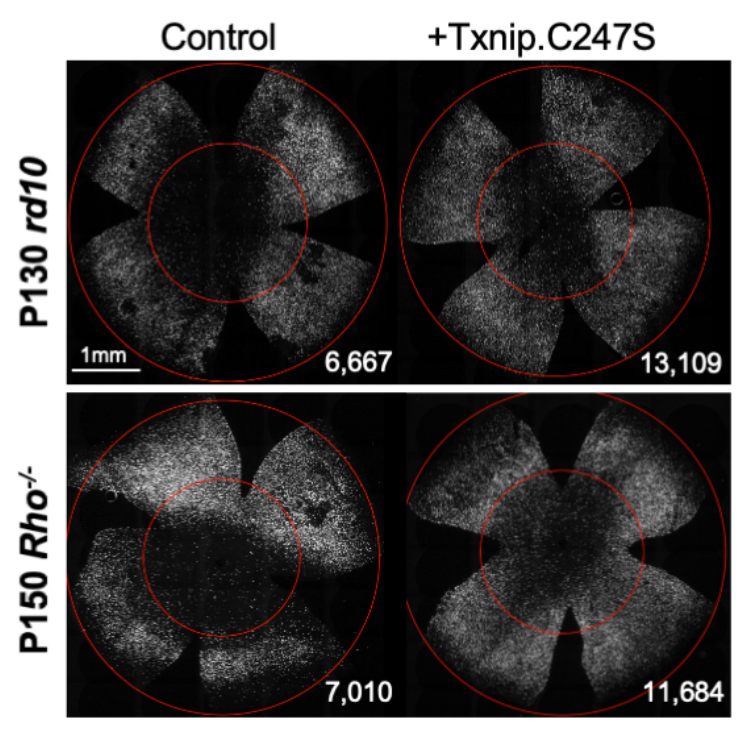

b

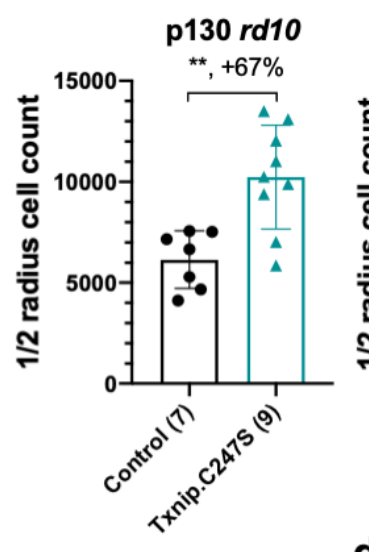

C

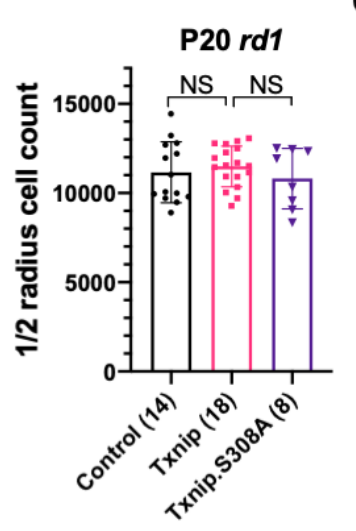

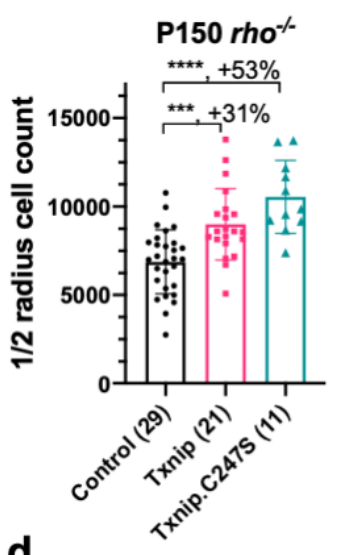

d

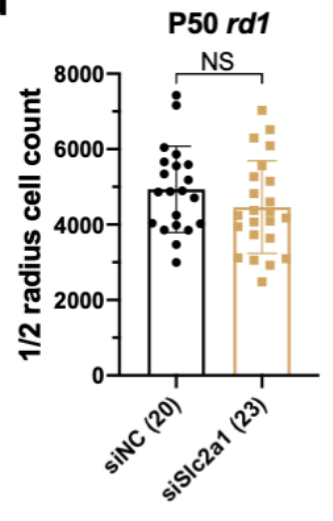




\section{Extended Data Fig. 3: Figures related to Fig. 3.}

a. AAV8-R01.7-Ldhb-FLAG with siNC control or Ldhb shRNAs in P21 wildtype (CD1) retina plus RedO-H2BGFP to track the infection. (Magenta: anti-FLAG. Green: anti-GFP. Gray: DAPI.)

b. Representative P50 rd1 flat-mounted retinas with H2BGFP (gray) labeled cones treated with Txnip + siNC, Txnip + siLdhb ${ }^{(\# 1)}$, or Txnip + siLdhb(\#3).

c. Quantification of H2BGFP-positive cones within the half radius of P50 rd1 retinas treated with Txnip + siNC, Txnip + siLdhb ${ }^{(\# 1)}$ or Txnip + siLdhb(\#3).

d. Representative P50 rd1 flat-mounted retinas with H2BGFP (gray) labeled cones treated with Txnip or Txnip + Ldha.

e. Quantification of H2BGFP-positive cones within the half radius of P50 rd1 retinas treated with Txnip or Txnip + Ldha. 
a

Ldhb-FLAG
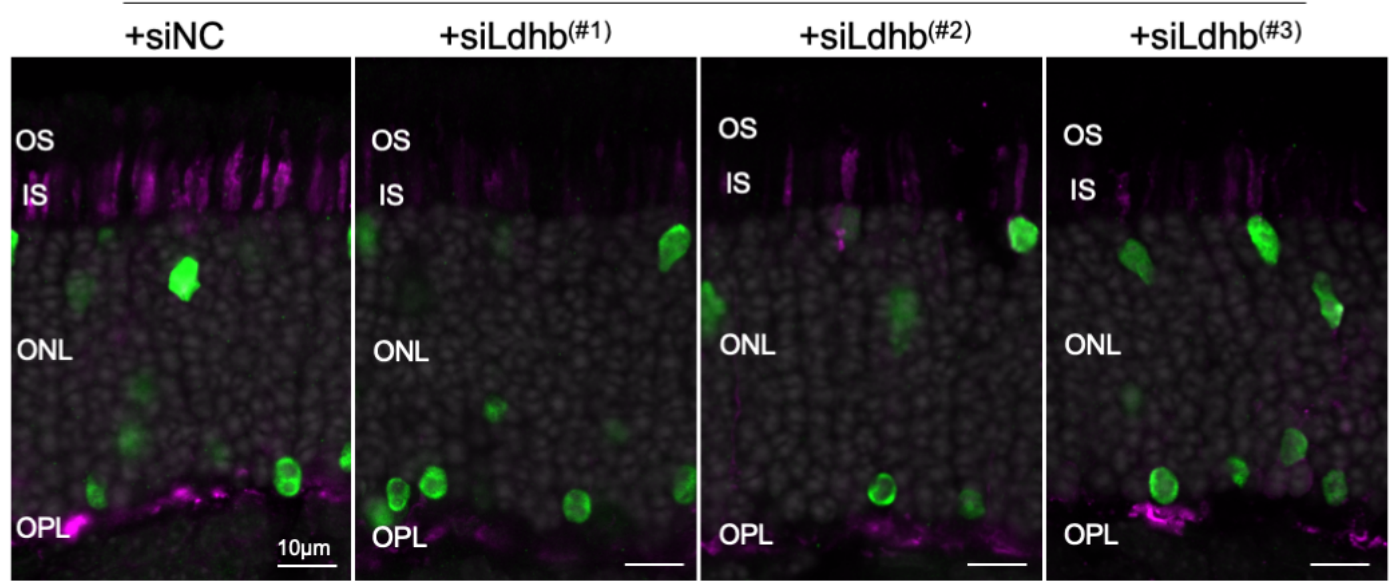

b

d
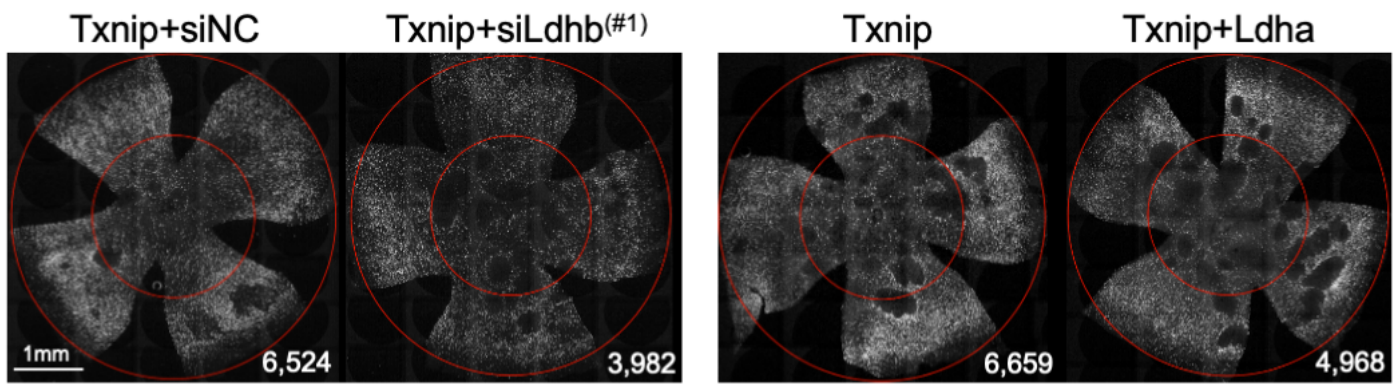

C

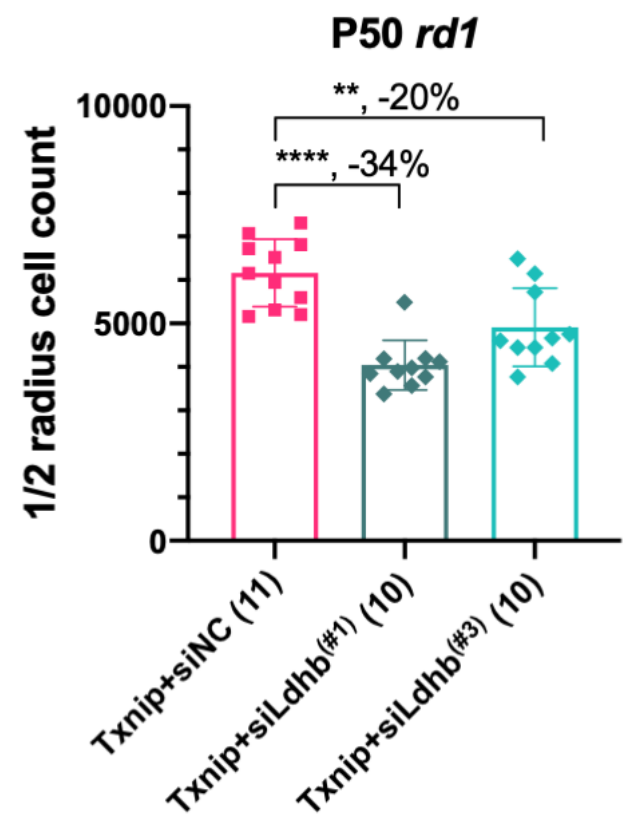

$\mathbf{e}$

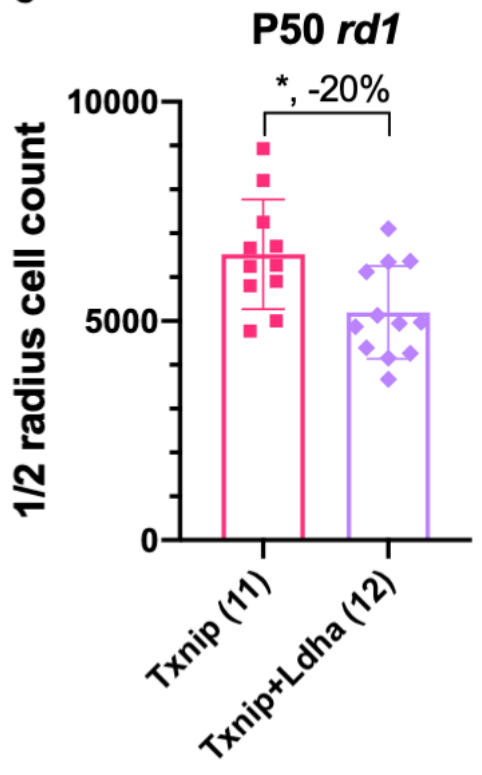




\section{Extended Data Fig. 4: Figures related to Fig. 4.}

a. Representative ex vivo live images of iGlucoSnFR labeled cones in P20 rd1 retinas cultured with high-glucose medium treated Txnip or control. (Green: glucose sensing GFP. Magenta: mRuby for self-normalization.)

b. Quantification of normalized iGlucoSnFR fluorescence intensity ( $F_{\text {iGlucoSnFR }}$ GFP : mRuby, proportional to glucose level) in cones from P20 rd1 retinas treated with Txnip or control ( $\approx 3$ images per retina).

c. ex vivo live images of pHRed labeled cones in P20 rd1 retinas cultured with high-glucose medium treated Txnip or control. (Magenta: fluorescence by $561 \mathrm{~nm}$ excitation, indicating a lower $\mathrm{pH}$. Green: fluorescence by $458 \mathrm{~nm}$ excitation, indicating a higher $\mathrm{pH}$.)

d. Quantification of normalized pHRed fluorescence intensity $\left(F_{p H R e d x} 561 \mathrm{~nm}: 458 \mathrm{~nm}\right.$, inversely proportional to $\mathrm{pH}$ value) in cones from $\mathrm{P} 20$ rd1 retinas treated with Txnip or control ( $\approx 3$ images per retina). 
a

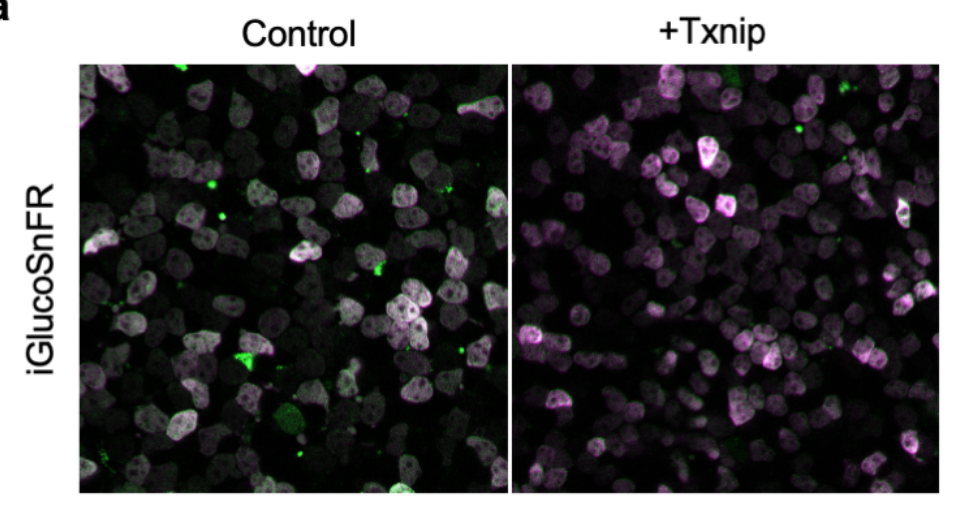

C

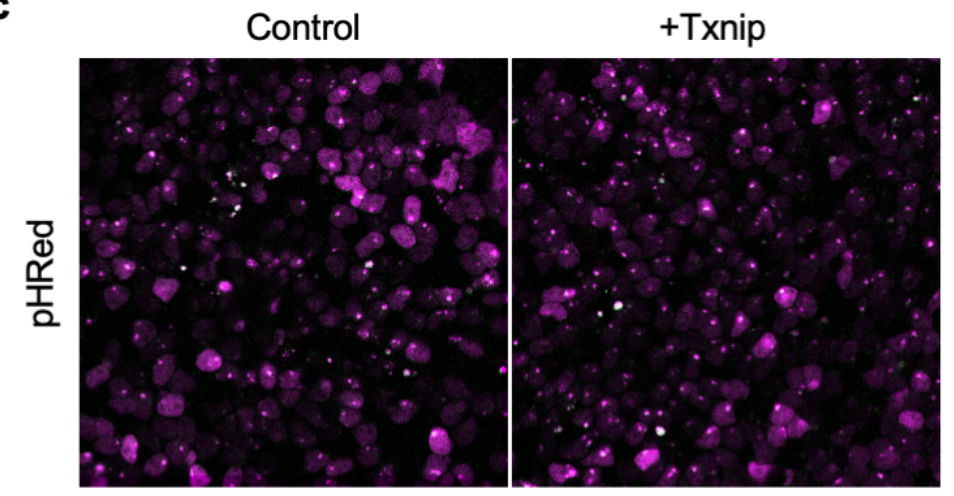

b

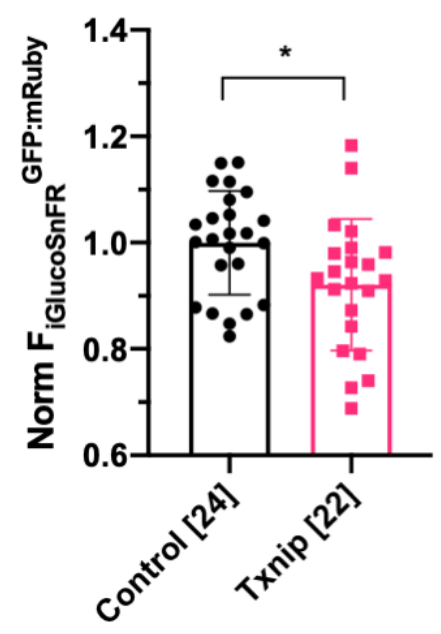

d

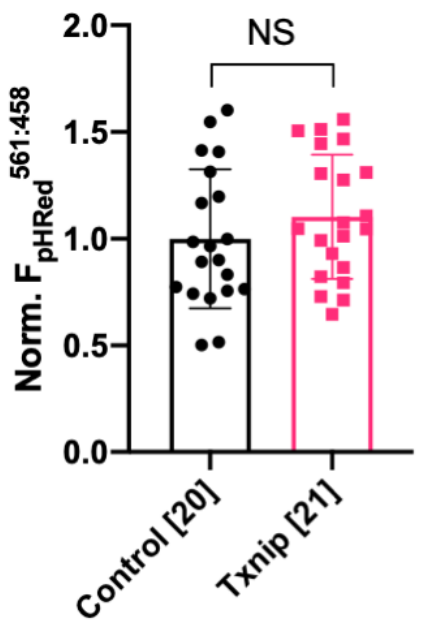




\section{Extended Data Fig. 5: Figures related to Fig. 5.}

a. Volcano plots of differentially expressed genes in RP cones FACS sorted from P21 rd1 retinas (left panel; +Txnip, $n=3$, relative to control, $n=6$ ) and $P 90$ rho $^{-1-}$ retinas (right panel; + Txnip, $n=4$, relative to control, $n=4$ ). Dotted lines indicate adjusted $p<0.05$ and log2 fold change $>0.5$.

b. ddPCR fold-changes of commonly upregulated mitochondrial ETC genes and genes not confirmed (i.e. Acs/3 and Ft/1) by Txnip overexpression in FACS sorted P21 rd1 cones.

c. First panel: AAV8-SynP136-mitoRFP expression in P26 wildtype (BALB/c) retina crosssection. (Magenta: mitoRFP. Green: PNA. Gray: DAPI.) Other three panels: representative mitoRFP images from the control, Txnip and Txnip.S308A of fixed P20 rd1 retina flatmounts near optic nerve head, reflecting the mitochondrial function. (Magenta: mitoRFP. Gray: H2BGFP, for infection normalization.)

d. Quantification of normalized mito-RFP:H2BGFP intensity of P20 parp1 retinas treated with control, Txnip or Txnip.S308A (4 images per retina).

e. Representative JC-1 dye staining image from live cones in P20 rd1 retina treated with Txnip + siNC or + siLdhb ${ }^{(\# 2)}$. (Magenta: J-aggregate, indicating high ETC function. Green: JC-1 monomer, for self-normalization. H2BGFP channel, the tracer of AAV infected area, is not shown.)

f. Quantification of normalized cone JC-1 dye staining (fluorescence intensity of Jaggregate:JC-1 monomer) from live cones in P20 rd1 retinas treated with Txnip + siNC or $\mathrm{siLdhb}^{(\# 2)}$ (4 images per retina).

g. Parp1 antibody staining of parp $1^{+/+}$(C57BL/6J) or parp $1^{-/-}$(on $129 \mathrm{~S}$ background) retina. (Magenta: Parp1. Gray: DAPI. Arrow heads: Parp1 staining from inner segments and cone nuclei).

h. Representative mitochondria EM images from P20 parp $1^{+/+}$or parp $1^{-/-} r d 1$ cones.

i. Quantification of mitochondrial diameters from P20 parp $1^{+/+}$or parp $1^{-/-} r d 1$ cones from one retina per condition. 
a

P21 rd1

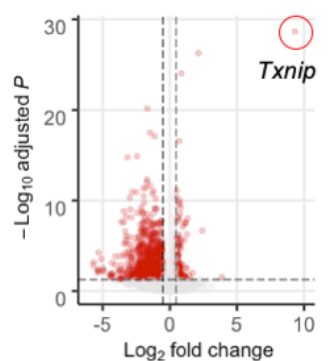

C

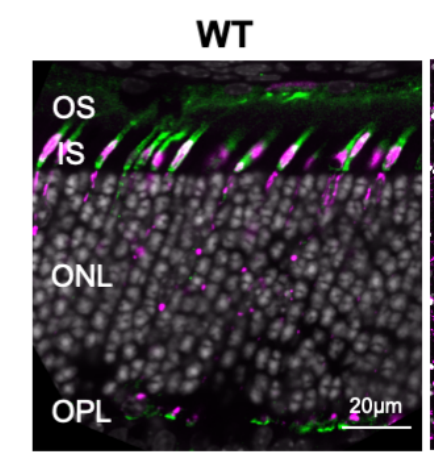

d

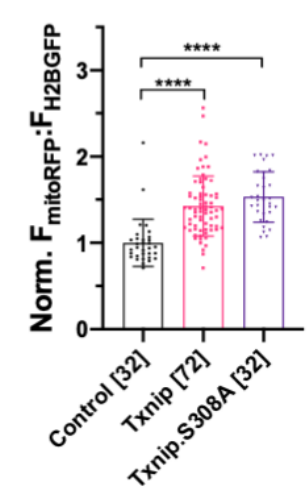

\section{e}

b

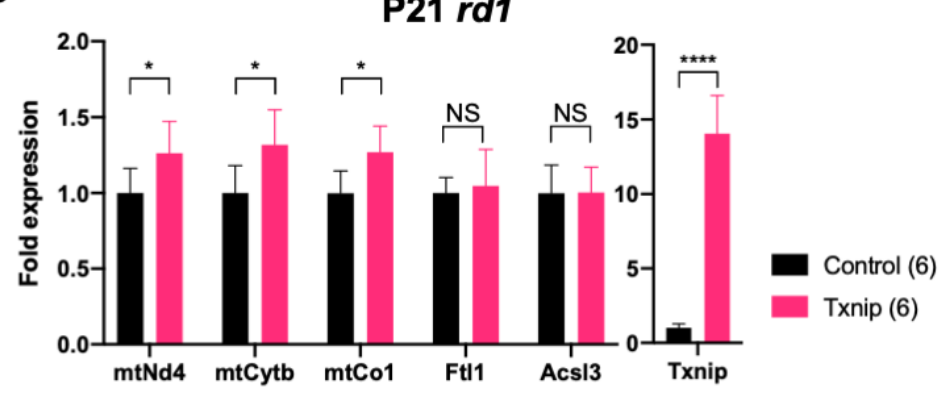

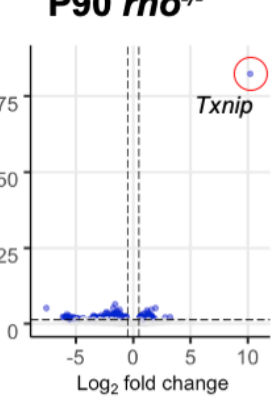

Control

P20 rd1
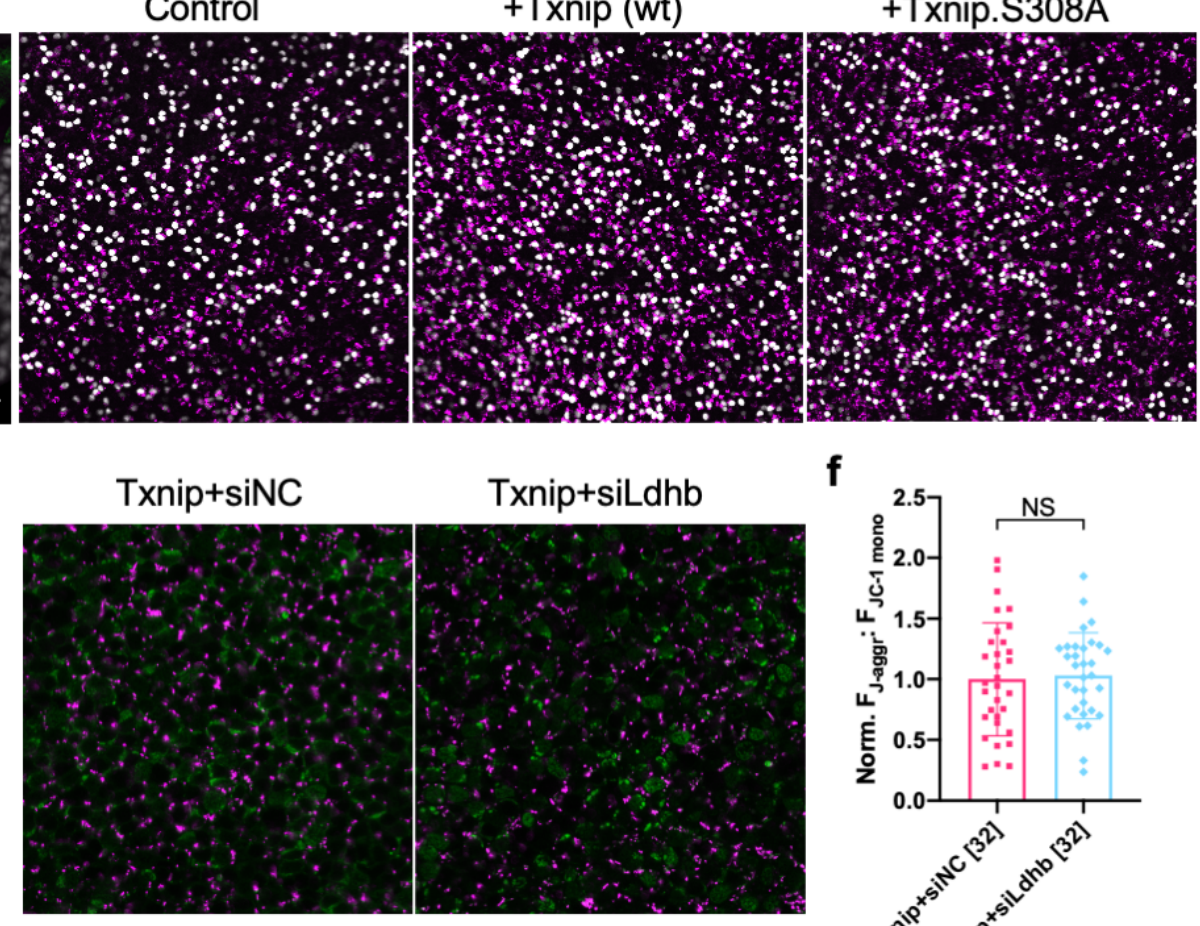

Txnip+siLdhb

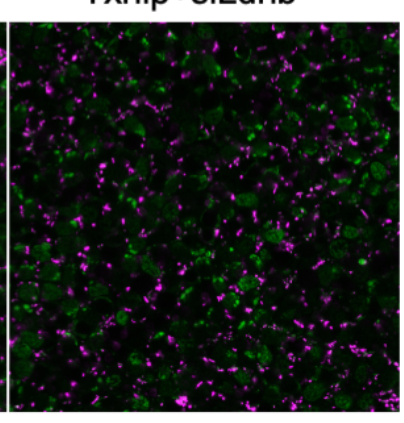

f

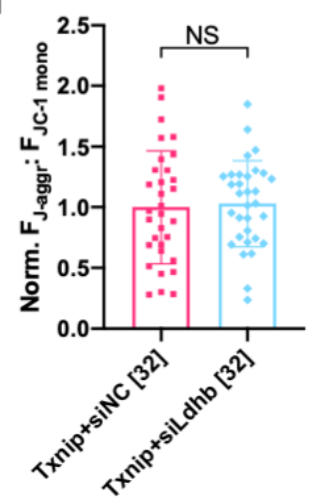

g
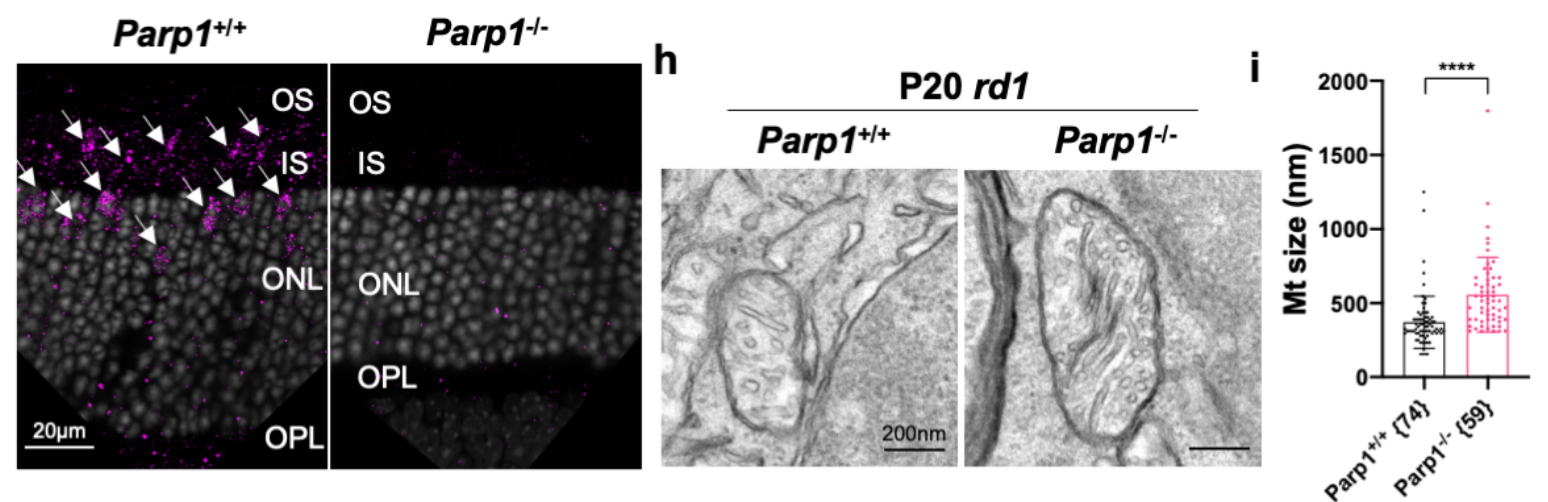
194 Extended Data Fig. 6: Figures related to Fig. 7 and 8.

a. Glut1 expression in P37 wildtype (C57BL/6J) eyes treated with control, AAV8-Best1-Txnip or AAV8-Best1-Txnip.C247S.LL351\&352AA (Magenta: Glut1. Green: RedO-H2BGFP for infection tracing, leaky expression in RPE due to recombination with Best1-vector due to unclear mechanism(Wu et al., n.d.). Gray: DAPI.)

b. Quantification of H2BGFP-positive cones within the half radius of P50 rd1 retinas treated with 6 different Best1-vectors or control. (Please note: we did not use dark-reared rd10 for testing the RdCVF vector, and our AAV capsid and promoter were different from the original study (Byrne et al., 2015).)

c. Quantification of H2BGFP-positive cones within the half radius of P50 rd1 retinas treated with Mpc1 + Mpc2 or control.

d. Representative P50 rd1 flat-mounted retinas with H2BGFP (gray) labeled cones treated with Vegf164 and control. Abbreviation: Txnip.CS.LLAA = Txnip.C247S.LL351\&352AA.

e. Quantification of $\mathrm{H} 2 \mathrm{BGFP}$-positive cones within the half radius of $\mathrm{P} 50 \mathrm{rd1}$ retinas treated with Vegf164 or control.

f. Quantification of H2BGFP-positive cones within the half radius of P50 $r d 1$ retinas treated with control, SynPVI-Hk2, SynPVI-Nrf2, RedO-Ldhb, RedO-Cx3cl1, RedO-Txnip and combinations with RedO-Txnip. Abbreviation: VI = SynPVI. RO- or R- = RedO-. 
bioRxiv preprint doi: https://doi.org/10.1101/2021.01.27.428411; this version posted January 27, 2021. The copyright holder for this preprint

a

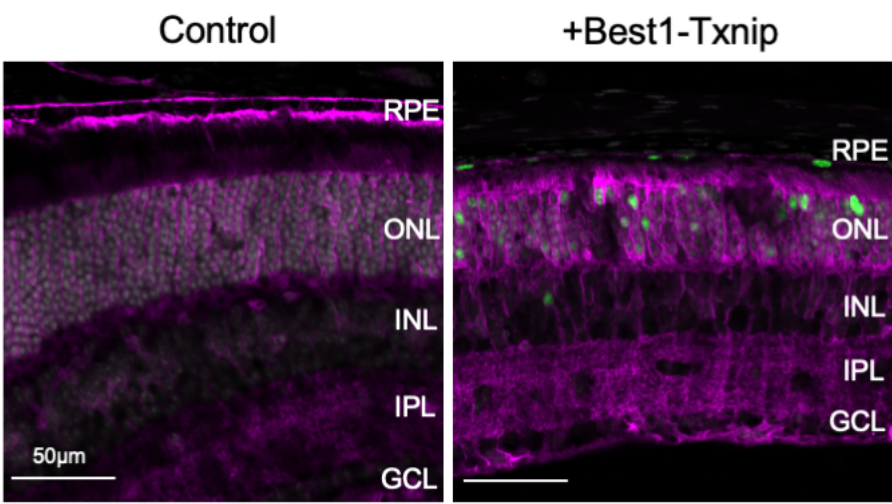

+Best1-Txnip.CS.LLAA

b

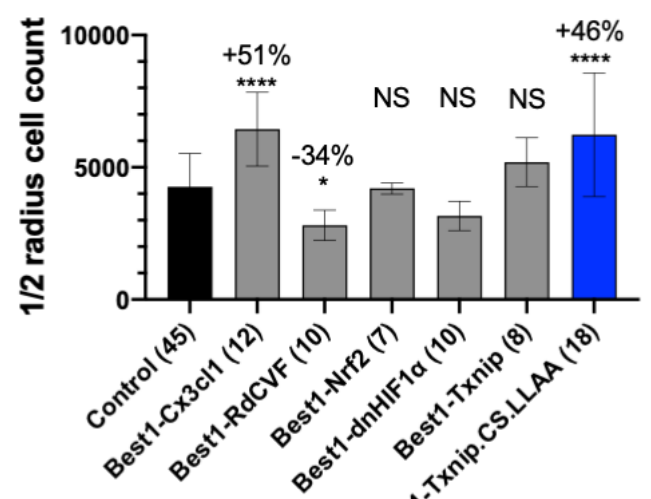

d

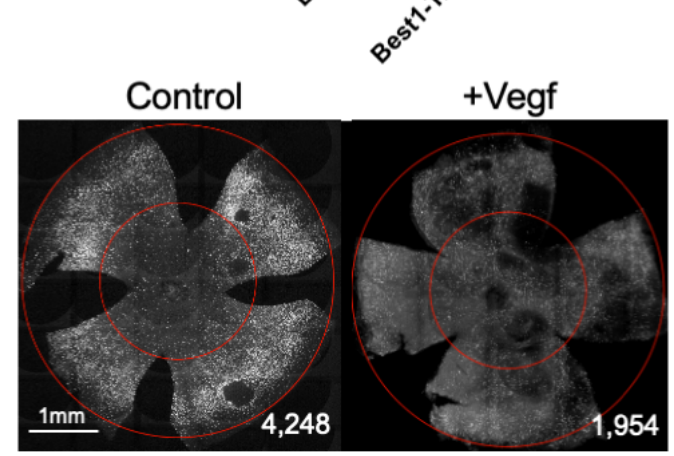

e
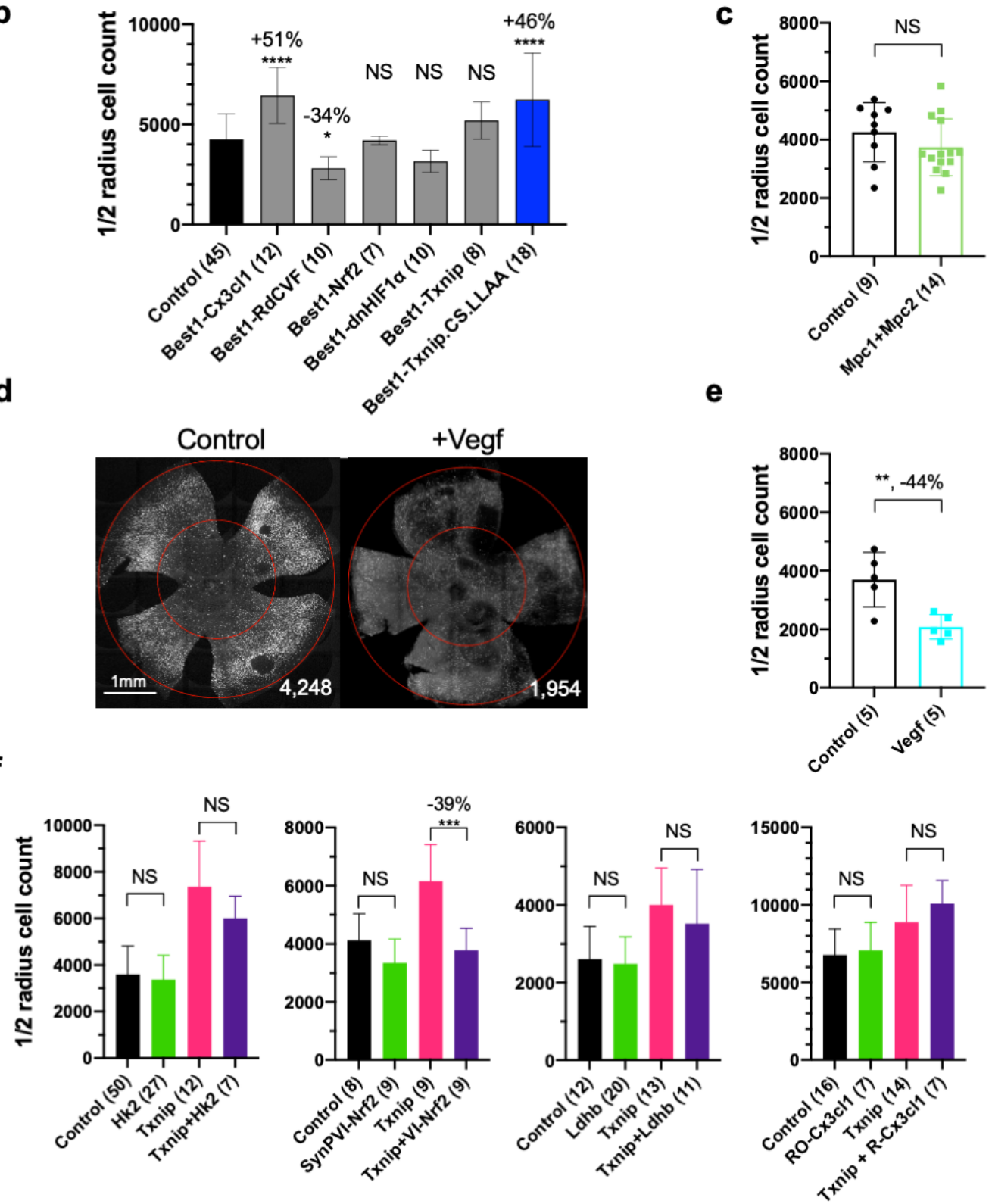
bioRxiv preprint doi: https://doi.org/10.1101/2021.01.27.428411; this version posted January 27, 2021. The copyright holder for this preprint (which was not certified by peer review) is the author/funder, who has granted bioRxiv a license to display the preprint in perpetuity. It is made available under aCC-BY 4.0 International license.

215 Extended Data Fig. 7: Schematics of proposed Txnip working mechanism. 
bioRxiv preprint doi: https://doi.org/10.1101/2021.0127.428411. this version posted January 27, 2021. The copyright holder for this preprint (which was not certified by peer review) is the author/funder, who has granted bioRxiv a license to display the preprint in perpetuity. It is made available under aCC-BY 4.0 International license.

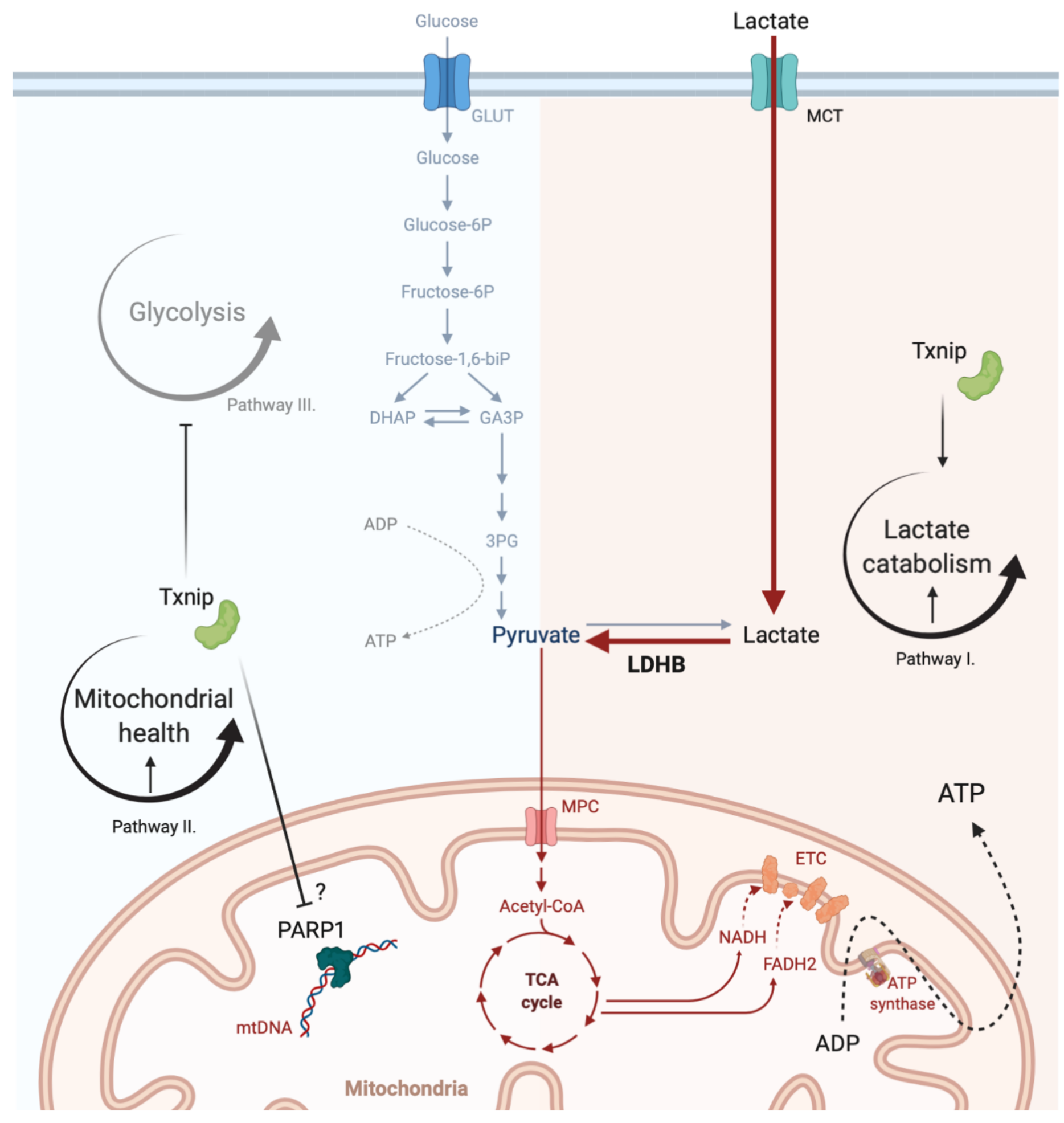


bioRxiv preprint doi: https://doi.org/10.1101/2021.01.27.428411; this version posted January 27, 2021. The copyright holder for this preprint (which was not certified by peer review) is the author/funder, who has granted bioRxiv a license to display the preprint in perpetuity. It is made available under aCC-BY 4.0 International license.

218 Extended Data Fig. 8: SIc2a1/Glut1 shRNA in vitro screening and screened out siSIc2a1(\#a) 219 for in vivo experiments.

a. GFP signals from overnight transfected HEK293T cells labeled with CAG-SIc2a1-IRESGFPd2 (CIGd2-Glut1) or CIGd2-Chx10 (negative control group) plus siSlc2a1(\#a, b, c, d) or siNC at $1: 1$ or $1: 2$ ratios.

b. mCherry signals (positive-control for transfection) from the same imaging regions as in subFig. a above. 
bioRxiv preprint doi: https://doi.org/10.1101/2021.01.27.428411; this version posted January 27, 2021. The copyright holder for this preprint (which was not certified by peer review) is the author/funder, who has granted bioRxiv a license to display the preprint in perpetuity. It is made available under aCC-BY 4.0 International license.

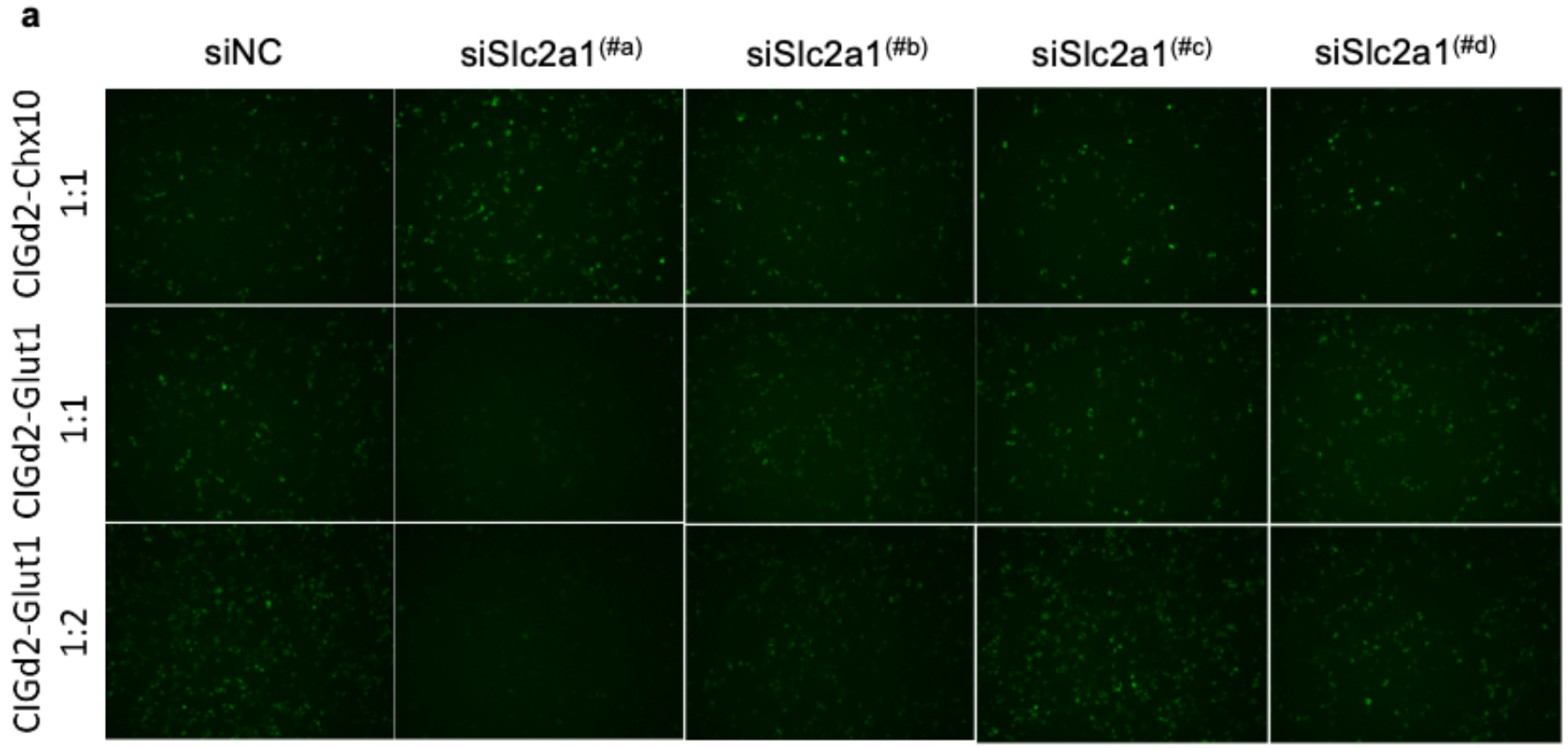

b

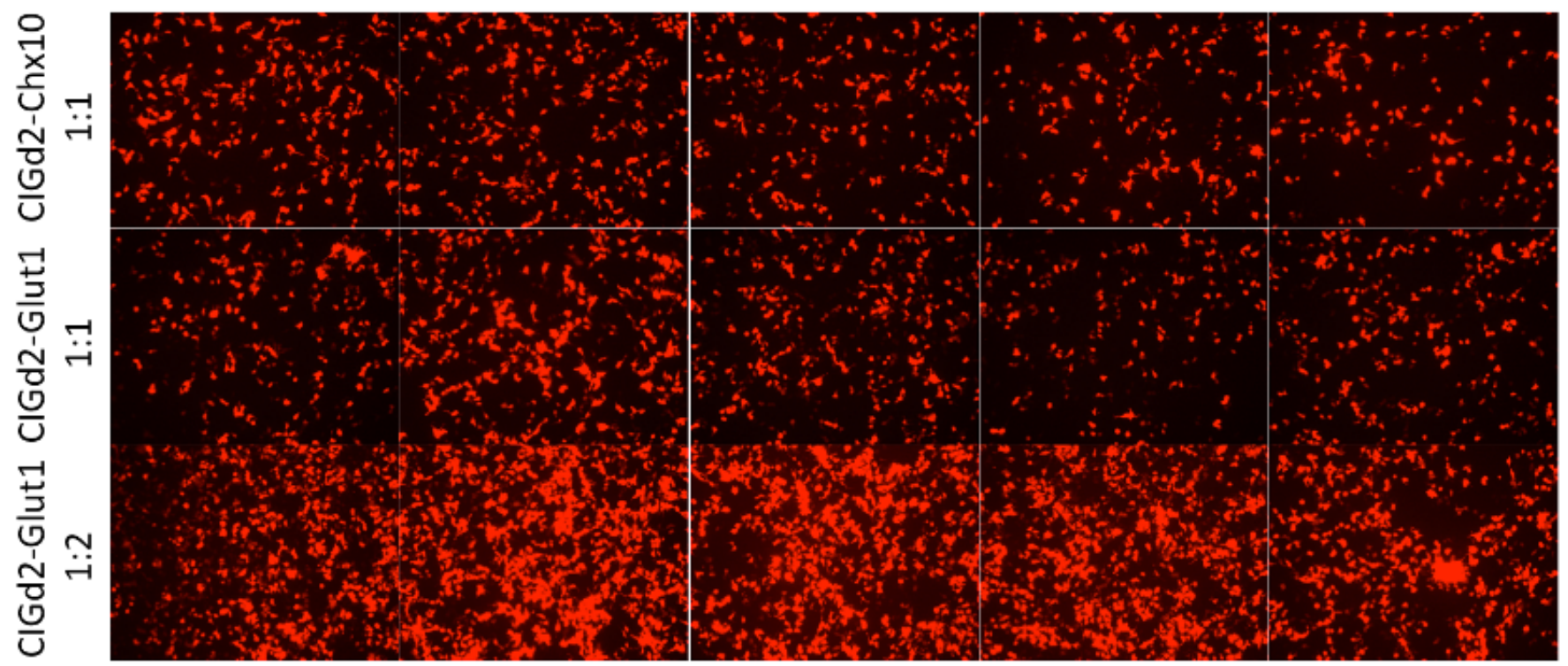


bioRxiv preprint doi: https://doi.org/10.1101/2021.01.27.428411; this version posted January 27, 2021. The copyright holder for this preprint (which was not certified by peer review) is the author/funder, who has granted bioRxiv a license to display the preprint in perpetuity. It is made available under aCC-BY 4.0 International license.

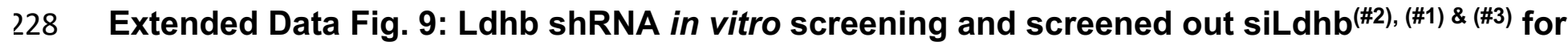
229 in vivo experiments

a. GFP signals from overnight transfected HEK293T cells labeled with CAG-Ldhb-IRESGFPd2 (ClGd2-Ldhb) or CIGd2-Chx10 (negative control group) plus siLdhb(\#1, 2, 3, 4) or siNC at $1: 1$ or $1: 2$ ratios.

b. mCherry signals (positive-control for transfection) from the same imaging regions as in subFig. a above. 
bioRxiv preprint doi: https://doi.org/10.1101/2021.01.27.428411; this version posted January 27, 2021. The copyright holder for this preprint (which was not certified by peer review) is the author/funder, who has granted bioRxiv a license to display the preprint in perpetuity. It is made available under aCC-BY 4.0 International license.
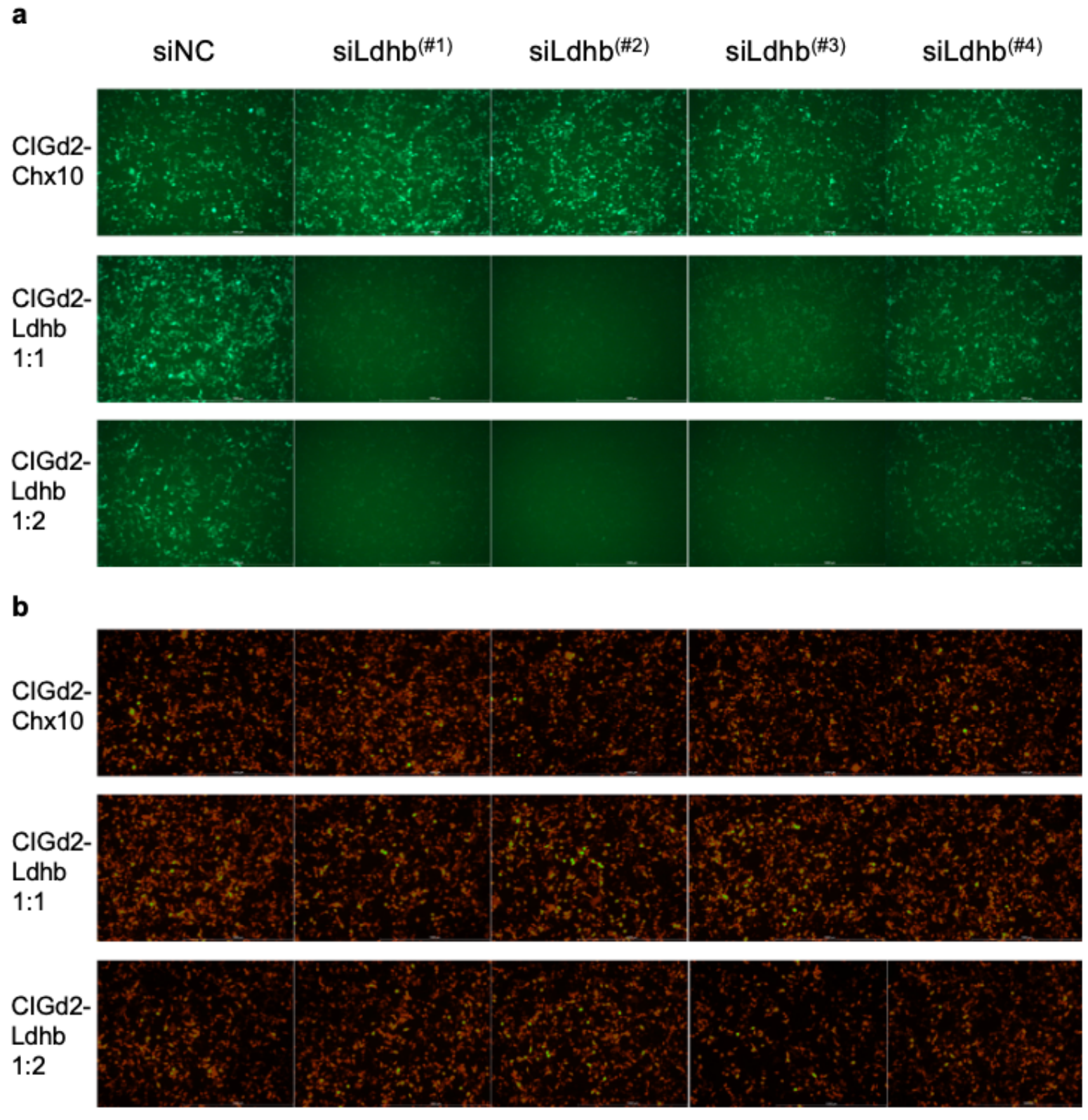
bioRxiv preprint doi: https://doi.org/10.1101/2021.01.27.428411; this version posted January 27, 2021. The copyright holder for this preprint (which was not certified by peer review) is the author/funder, who has granted bioRxiv a license to display the preprint in perpetuity. It is made available under aCC-BY 4.0 International license.

239 Extended Data Fig. 10: Oxct1 shRNA in vitro screening and screened out siOxct1 ${ }^{(\# c)}$ for in $240 \quad$ vivo experiments

a. GFP signals from overnight transfected HEK293T cells labeled with CAG-Oxct1-IRESGFPd2 (CIGd2-OXCT1) or CIGd2-Chx10 (negative control group) plus siOxct1(\#a, b, c) or siNC at $1: 2$ ratios.

b. mCherry signals (positive-control for transfection) from the same imaging regions as in subFig. a above. 
bioRxiv preprint doi: https://doi.org/10.1101/2021.01.27.428411; this version posted January 27, 2021. The copyright holder for this preprint (which was not certified by peer review) is the author/funder, who has granted bioRxiv a license to display the preprint in perpetuity. It is made available under aCC-BY 4.0 International license.

a

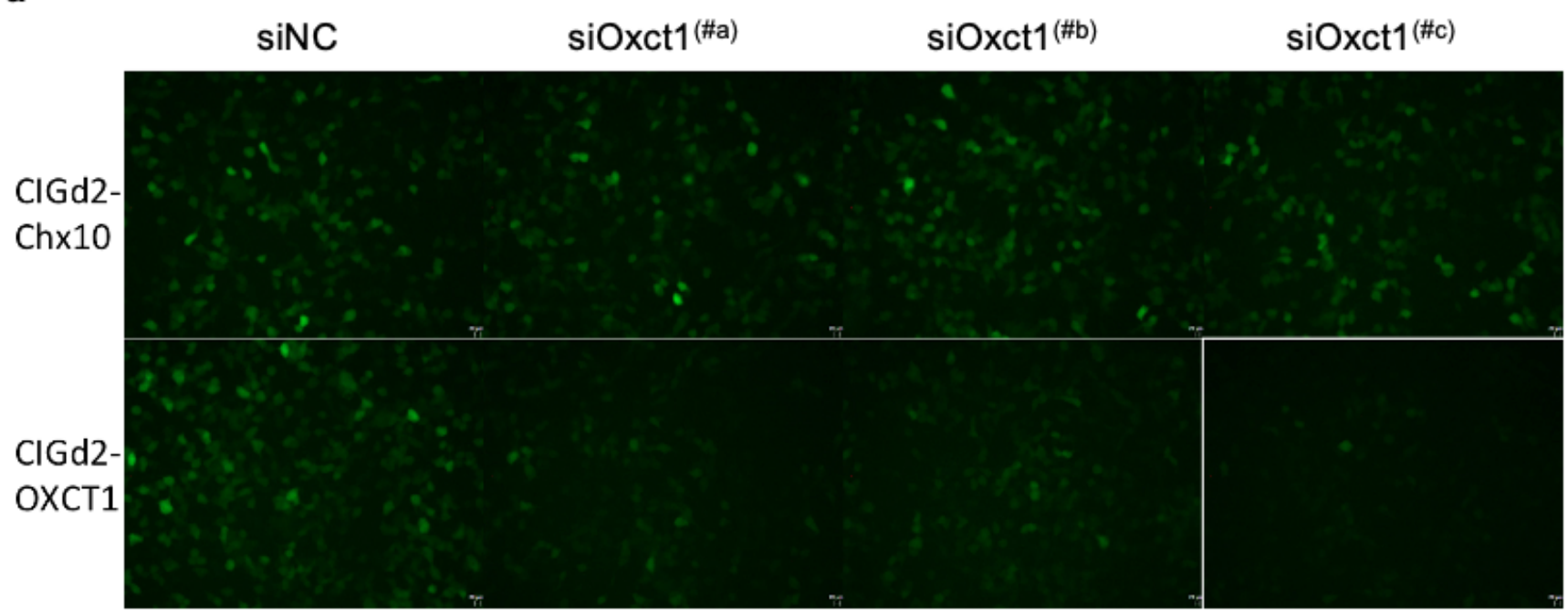

b

\section{CIGd2-}

Chx10

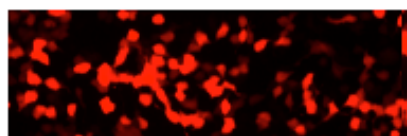

$\cdot 8 \cdot 4+200$

CIGd2-
OXCT1 
bioRxiv preprint doi: https://doi.org/10.1101/2021.01.27.428411; this version posted January 27, 2021. The copyright holder for this preprint (which was not certified by peer review) is the author/funder, who has granted bioRxiv a license to display the preprint in perpetuity. It is made available under aCC-BY 4.0 International license.

250 Extended Data Fig. 11: Cpt1a shRNA in vitro screening and screened out siCpt1 ${ }^{(\# c)}$ for in 251 vivo experiments

a. GFP signals from overnight transfected HEK293T cells labeled with CAG-Cpt1a-IRESGFPd2 (CIGd2-CPT1A) or CIGd2-Chx10 (negative control group) plus siCpt1a(\#a, b, c) or siNC at $1: 2$ ratios.

b. mCherry signals (positive-control for transfection) from the same imaging regions as in subFig. a above. 
bioRxiv preprint doi: https://doi.org/10.1101/2021.01.27.428411; this version posted January 27, 2021. The copyright holder for this preprint (which was not certified by peer review) is the author/funder, who has granted bioRxiv a license to display the preprint in perpetuity. It is made available under aCC-BY 4.0 International license.

a

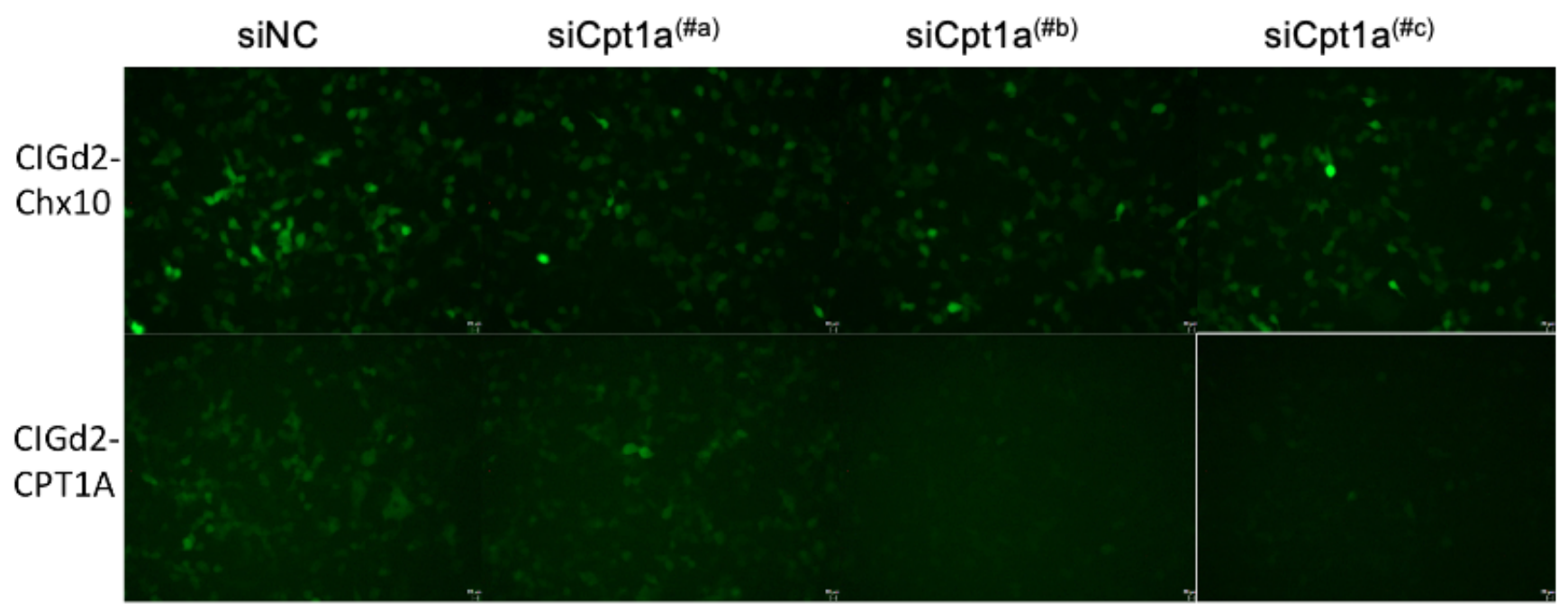

b

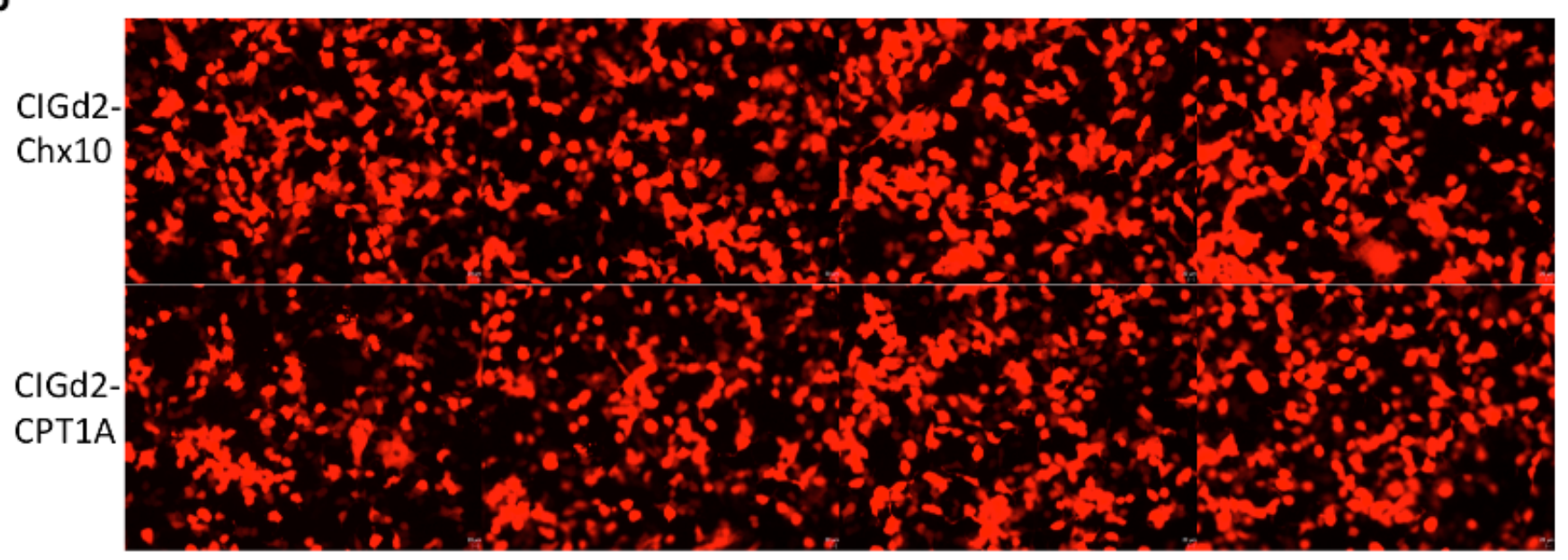

